University of Louisville

ThinkIR: The University of Louisville's Institutional Repository

$12-2013$

\title{
A semi-empirical Hamiltonian for boron, phosphorus and compounds containing boron, phosphorus and silicon.
}

Paul S. Tandy

University of Louisville

Follow this and additional works at: https://ir.library.louisville.edu/etd

\section{Recommended Citation}

Tandy, Paul S., "A semi-empirical Hamiltonian for boron, phosphorus and compounds containing boron, phosphorus and silicon." (2013). Electronic Theses and Dissertations. Paper 1417.

https://doi.org/10.18297/etd/1417

This Doctoral Dissertation is brought to you for free and open access by ThinkIR: The University of Louisville's Institutional Repository. It has been accepted for inclusion in Electronic Theses and Dissertations by an authorized administrator of ThinkIR: The University of Louisville's Institutional Repository. This title appears here courtesy of the author, who has retained all other copyrights. For more information, please contact thinkir@louisville.edu. 


\title{
A SEMI-EMPIRICAL HAMILTONIAN FOR BORON, PHOSPHORUS AND COMPOUNDS CONTAINING BORON, PHOSPHORUS AND SILICON
}

\author{
By \\ Paul S. Tandy \\ B.S. PHYSICS, University of Louisville, 2007 \\ M.S. PHYSICS, University of Louisville, 2007 \\ M.S. ELECTRICAL ENGINEERING, University of Louisville, 2010 \\ A Dissertation \\ Submitted to the Faculty of the \\ College of Arts and Sciences of the University of Louisville \\ in Partial Fulfillment of the Requirements \\ for the Degree of
}

Doctor of Philosophy

Department of Physics and Astronomy

University of Louisville

Louisville, Kentucky

December 2013 
Copyright 2013 by Paul S. Tandy

All rights reserved 

A SEMI-EMPIRICAL HAMILTONIAN FOR BORON, PHOSPHORUS AND COMPOUNDS CONTAINING BORON, PHOSPHORUS AND SILICON

By

Paul S. Tandy

B.S. PHYSICS, University of Louisville, 2007

M.S. PHYSICS, University of Louisville, 2007

M.S. ELECTRICAL ENGINEERING, University of Louisville, 2010

A Dissertation Approved on

December 5, 2013

By the following Dissertation Committee

$\overline{\text { Shi-Yu Wu, PhD Dissertation Director }}$

Chakram Jayanthi, PhD

Ming Yu, PhD

Gamini Sumanasekera, PhD

Robert Cohn, PhD 


\section{DEDICATION}

I dedicate this to August, Penelope, Leo, and Heather Tandy for their love and support over the many years. Without them, I would have nothing. 


\section{ACKNOWLEDGMENTS}

I would like to thank the faculty of the Physics and Electrical Engineering departments of the University of Louisville for all the conversations, help, advice, and support during my graduate studies at the University of Louisville. In particular I would like to greatly thank Professors Shi-Yu Wu and Chakram Jayanthi, for their continuous support, help, aid and guidance over the many years. Extremely special thanks to Professor Ming Yu for her strong ability to interact and guide students in an effective manner. Many hours on many days I have worked with her on many occasions trying to solve difficult problems patiently and carefully. It is rare to see this type of generosity in a person, and it is much appreciated.

- Thesis committee - I would like to thank Dr. Ming Yu, Dr. Robert Cohn, Dr. Gamini Sumanesekera, Dr. Chakram Jayanthi, and Dr. Shi-Yu Wu for their great patience and understanding. Their advice over the years has been nothing but stellar, and I thank them greatly for reading and helping me edit my dissertation.

- Dillon Fellowship - Without this funding effort I could not have finished my PhD. I am greatly indebted to the Dillon family and was very lucky to have known Dr. Dillon before he passed away. He was a great addition to the world and has left a great legacy. 
- I would like to make a special thanks to Dr. Christopher Davis for personally handling the difficulties involved with me as a graduate student as Program Director in the Department of Physics.

- I must thank my colleagues for endless conversations advice and help. This includes the esteemed Dr. Chris Leahy, Mr. Harrison Simrall, Mr. Lyle Smith, Dr. Alex Tchernatinsky, Dr. Dmitri B. Migas, Dr. Indira Chaudhuri, Dr. Jeff Hay, Dr. Wei Tian, Dr. Yufeng Zhao, Mr. Sanguo Shen, Mr. Cherno Kah, and many others over the years, too numerous to count.

- Special thanks go to Chris Leahy, Harrison Simrall and Lyle Smith. They were my office mates for years, and I could not have asked for a better set of people to work with. They made my day many times, and the laughs will never be forgotten. I would like to give a special thanks to Dr. Chris Kimmer for his guidance and advice.

- I would like to thank the Department of Physics and Department of Electrical and Computer Engineering for many exciting and stimulating discussions over the many years.

- Family and friends - l'd like to thank all my family and friends for being there this past 9 years as I pursued my education. I can honestly say that their patience and support has been the driving force in me. 


\title{
ABSTRACT
}

\section{A SEMI-EMPIRICAL HAMILTONIAN FOR BORON, PHOSPHORUS AND COMPOUNDS CONTAINING BORON, PHOSPHORUS AND SILICON}

\author{
Paul Tandy
}

\section{December 5, 2013}

In this work, a 25 parameter semi-empirical Hamiltonian for boron and phosphorus is developed. The Hamiltonian contains both environmentdependent terms and electron-correlation terms with the on-site charge calculated self-consistently. One of the goals of this work is to obtain the parameterized Hamiltonian for boron and phosphorus by fitting the properties of small boron and phosphorus clusters and the bulk phases, as obtained by our method, to ab-initio calculations. The general structure of this Hamiltonian and all phenomenological functions contained within have been successful in predicting the properties of intermediate silicon clusters as well as extended structures of silicon with great precision. Large nanostructures and phosphorus and boron doped silicon structures are explored. 


\section{TABLE OF CONTENTS}

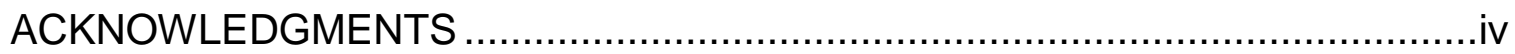

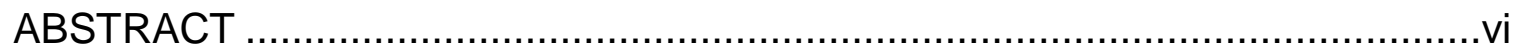

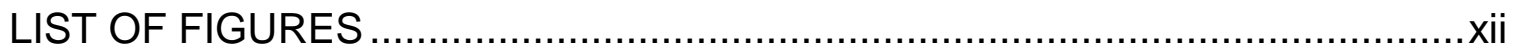

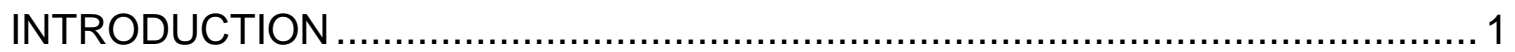

CHAPTER I: DESIGN OF A MODEL HAMILTONIAN AND OVERLAP ............. 6

1.1 GENERAL STRUCTURE OF THE HAMILTONIAN $\ldots \ldots \ldots \ldots \ldots \ldots \ldots \ldots \ldots \ldots$

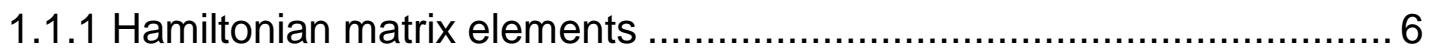

1.1.2 Interaction Terms and modeling ....................................... 10

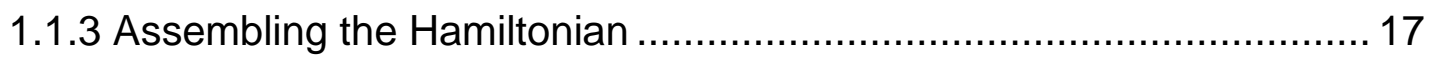

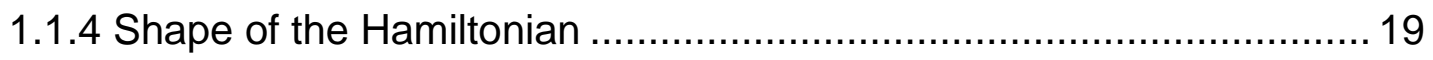

1.2 GENERAL STRUCTURE OF THE OVERLAP AND ENERGY .............. 20

1.2.1 Limit Requirements of the Phenomenological functions .................. 20

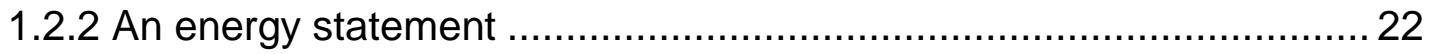

CHAPTER II: BORON PARAMETER ACQUISTION ................................ 25

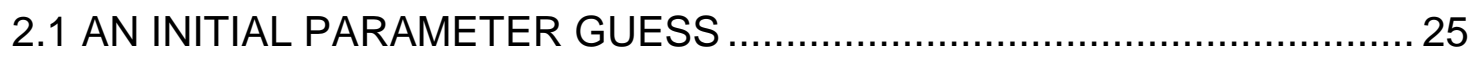

2.1.1 Initial Overlap Parameter Estimates and Constraints ...................... 26 
2.1.2 Initial Hamiltonian Parameter Estimates and Constraints

2.2.1 Convenient fit properties

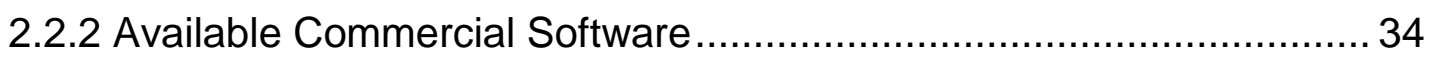

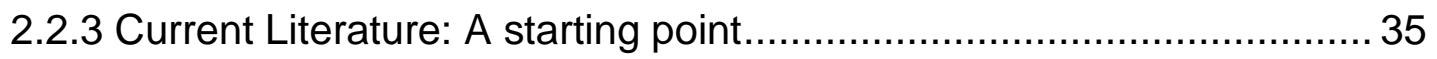

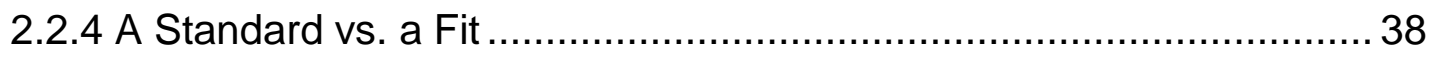

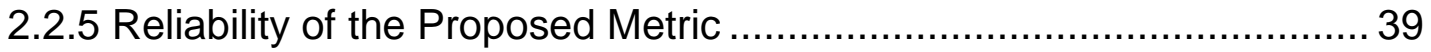

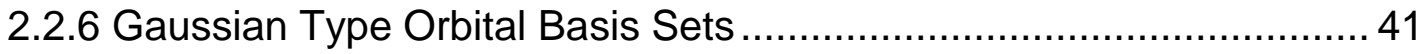

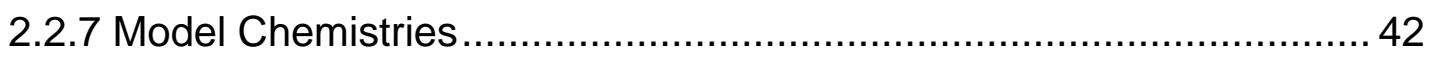

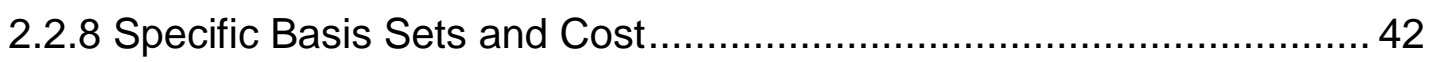

2.2.9 Fixing Symmetry for clusters ..................................................... 43

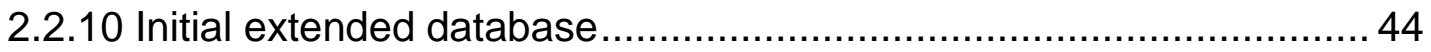

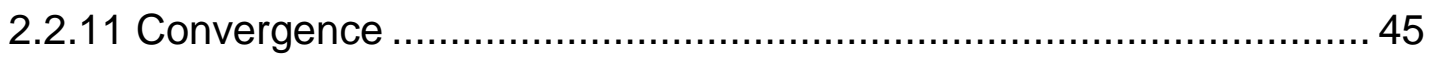

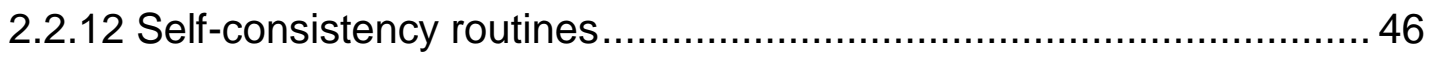

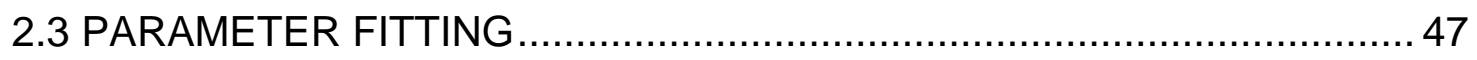

2.3.1 Stage 1: A small cluster database …............................................. 47

2.3.2 Stage 2: Addition of a fictitious sets of extended phases....................51

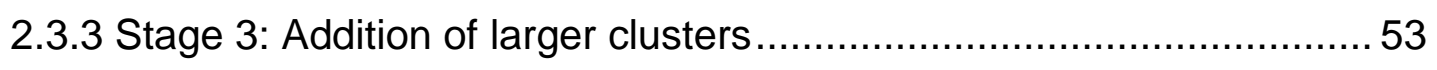

2.3.4 Stage 4: Inclusion of laboratory synthesized bulk phases ................. 55

2.3.5 Stage 5: Removal of fictitious phases........................................... 57 
2.3.6 Stage 6: From density functional theory to coupled cluster theory .....58

2.3.7 Stage 7: Heavy weighting on the boron 12 cluster ...........................59

2.3.8 Stage 8: Addition of two dimensional extended phases ....................61

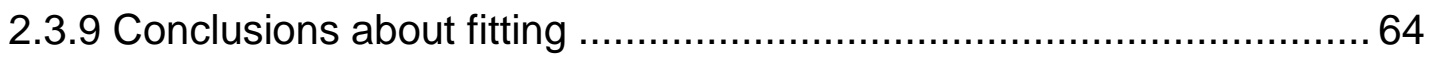

2.4 MOLECULAR DYNAMICS ROBUSTNESS CHECK ................................66

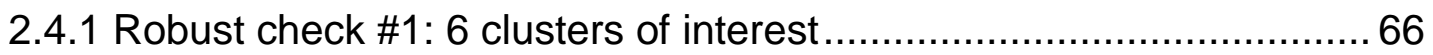

2.4.2 Robust check \#2: three isomers of the 13 atom boron ...................... 68

2.4.3 Robust check \#3: two isomers of the 15 atom boron.......................... 69

2.4.4 Robust check \#4: reconstruction of the triangular to buckled sheet....69

2.4.5 Robust check \#5: Energy ordering of all sheets and conclusions....... 71

CHAPTER III: BORON SIMULATIONS …................................................... 73

3.1 TWO DIMENSIONAL SHEETS ....................................................... 73

3.2 THE BORON 80 ATOM FULLERENE STRUCTURE ........................... 73

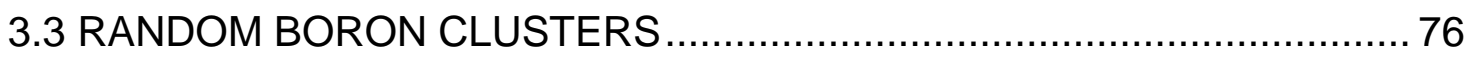

3.4 ALPHA BORON CUT CLUSTERS: RHOMBOHEDRAL SYMMETRY ...... 78

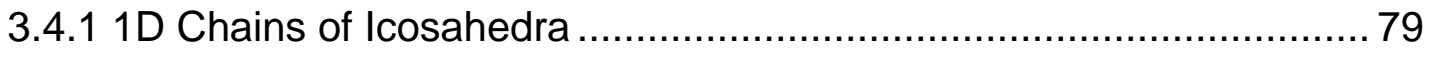

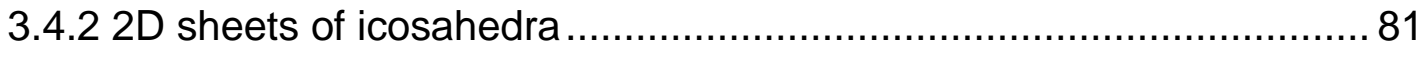

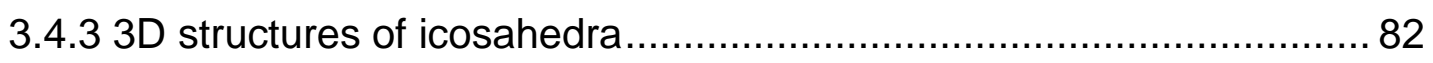

3.4.4 3D structures of icosahedra with equal aspect ration ........................ 84

3.5 ALPHA BORON CUT CLUSTERS: SPHERICAL SYMMETRY ............... 85 
3.5.1 Spherical cuts of alpha boron 85

3.5.2 Trimmed spherical cuts of alpha boron.......................................... 90

3.6 LOCAL ANALYSIS OF BORON CLUSTERS OF SIMILAR SIZE .............93

CHAPTER IV: PHOSPHORUS PARAMETER ACQUISITION ......................... 98

4.1 PARAMETER FITTING OF PHOSPHORUS ….................................. 98

4.1.1 Cluster set used in the fitting of phosphorus ................................... 98

4.1.2 Black phosphorus: An extended phosphorus phase ......................... 99

4.1.3 Optimized phosphorus overlaps ................................................. 100

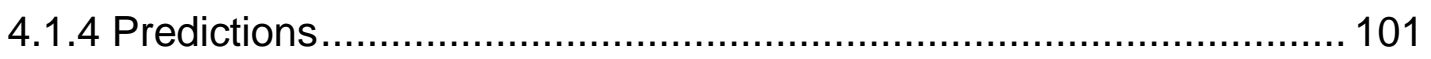

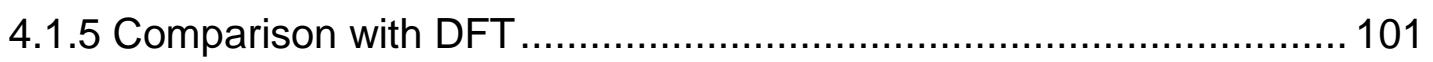

4.1.6 Conclusions about the final parameters set.................................. 102

CHAPTER V: DOPING THE SILICON WIRE WITH BORON AND

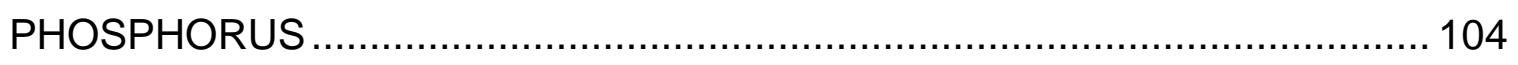

5.1 THE HETEROGENEOUS HAMILTONIAN AND OVERLAP ...................104

5.1.1 Parameter based weighted averaging .......................................... 107

5.1.2 Function based weighted averaging ............................................ 107

5.1.3 Parameter based onsite energy weighted averaging ...................... 107

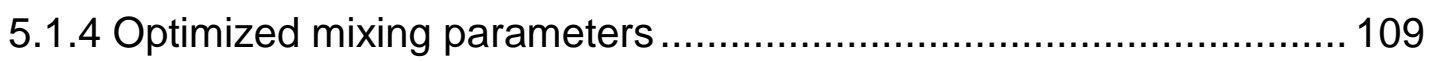

5.2 DOPING THE PERPENDICULAR WIRE ...................................... 110

5.2.1 Hydrogen passivated multi-twin wire with boron doping .................. 113 
5.2.2 Simultaneous Boron and Phosphorus Doping .............................. 118

CHAPTER VI: SUMMARY AND FUTURE WORK........................................ 124

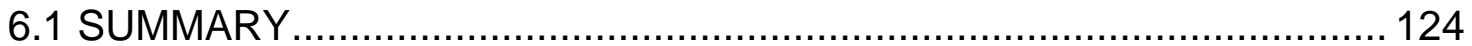

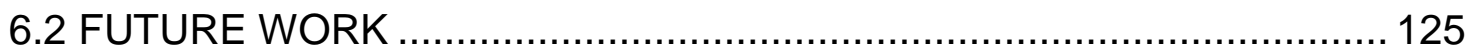

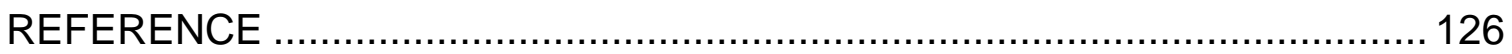

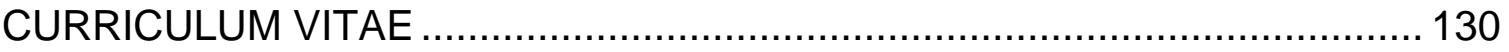




\section{LIST OF FIGURES}

Figure 1. Onsite model approximations ........................................................... 12

Figure 2. DFT overlap for boron dimer using a STO-3G basis set.................... 27

Figure 3. DFT overlap for boron dimer using a CEP-4G basis set.................... 28

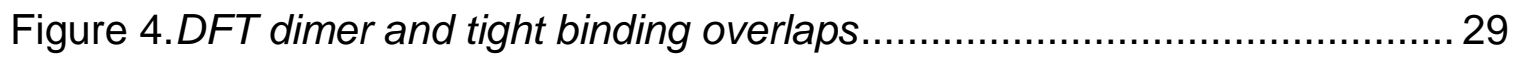

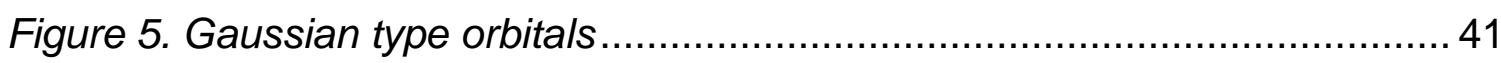

Figure 6. fixed symmetry during the geometry optimization ............................ 43

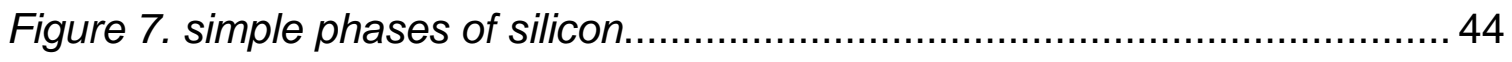

Figure 8. binding energy vs. relative atomic volume for boron ........................ 45

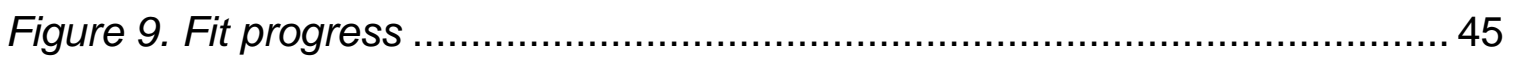

Figure 10. Self-consistency methodology …………...................................... 46

Figure 11. Clusters initially used for fitting .................................................... 49

Figure 12. Overlap functions of boron (optimized shown) …........................... 50

Figure 13. General shape of the Hamiltonian ................................................ 51

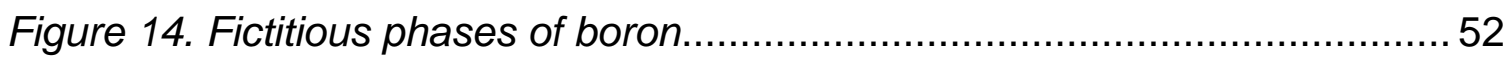

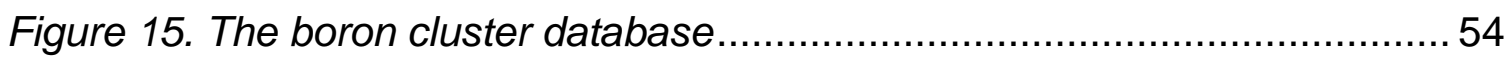

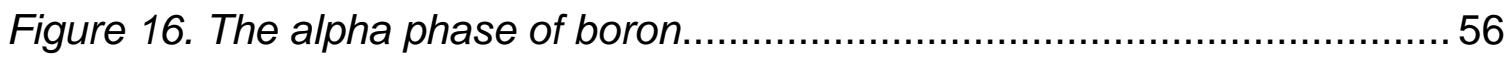

Figure 17. An image of boron alpha (rhombohedra) ......................................5 57

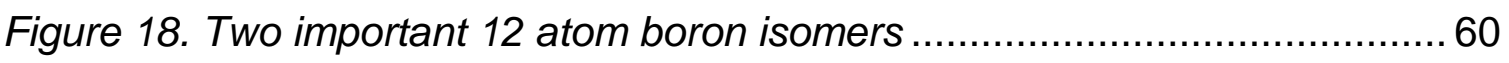

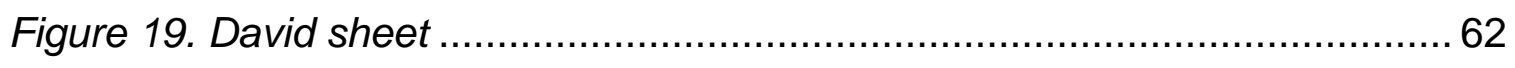


Figure 20. DFT results of 2D boron sheets (Yu Ming) ..................................... 63

Figure 21. Fit quality of extended phases.................................................... 64

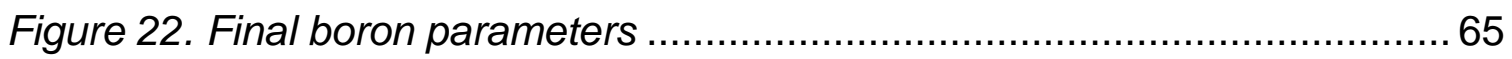

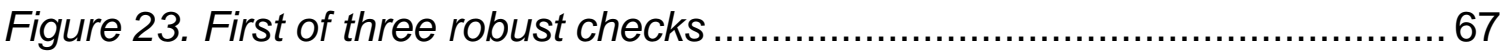

Figure 24. Three isomers of 13 atom boron ............................................... 68

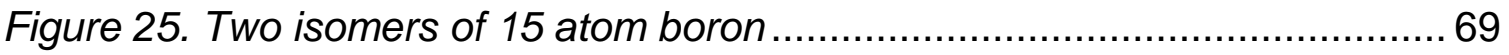

Figure 26. Reconstruction of the triangular to buckled sheet............................. 70

Figure 27. Energy ordering of all examined boron sheets ............................. 71

Figure 28. The B80 boron Ih, Th, and C2h fullerene from left to right................ 75

Figure 29. Density of states plot and homo lumo gap................................... 76

Figure 30. Random clusters vs. cuts and B80 …...................................... 77

Figure 31. Typical amorphous random boron cluster ................................... 78

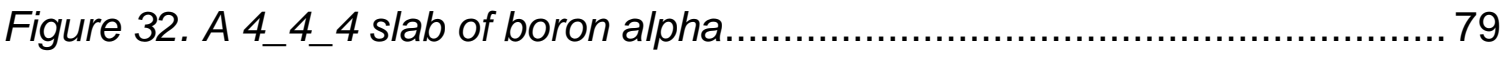

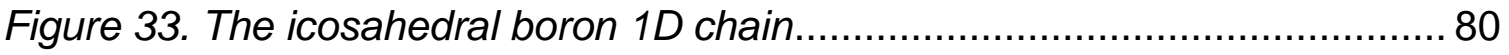

Figure 34. Energy plot of an icosahedral 1D chain as a function of length......... 80

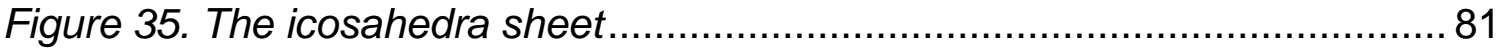

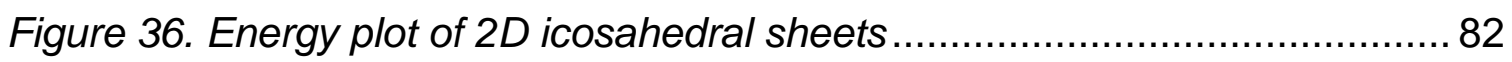

Figure 37. Rhombohedra cuts of alpha boron .............................................. 83

Figure 38. HOMO-LUMO gaps for select 3D rhombohedral cuts of alpha boron 83

Figure 39. Equi-cuts of boron alpha with rhombohedral symmetry ................... 85

Figure 40. Red is centered on ball, Blue is centered between balls .................. 86

Figure 41. Examples of large/small spherical cuts before and after relaxation ... 87

Figure 42. Energy plot of boron alpha spherical plots..................................... 89 
Figure 43. Plot of all spherical cuts homo-lumo gaps .... 90

Figure 44. Trimmed spherical cut of alpha boron

Figure 45. All alpha boron cuts, random clusters, bulk boron alpha, and the B80 buckyball. 92

Figure 46. Energy/atom of select clusters of size 220 atoms ....................... 93

Figure 47. Pair Distribution function for the 5 test clusters. ............................. 94

Figure 48.Angular distribution function for the 5 test clusters......................... 96

Figure 49. Clusters used in the phosphorus fitting process ............................. 99

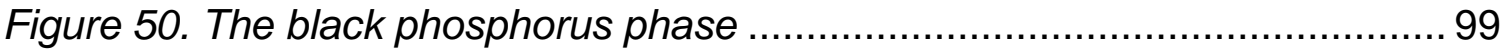

Figure 51. Overlap curves for phosphorus ............................................... 100

Figure 52. Predicted clusters from the phosphorus parameter set ................. 101

Figure 53. Comparison of binding energy with DFT and final parameter set.... 102

Figure 54. Optimized Phosphorus parameters shown in comparison to boron

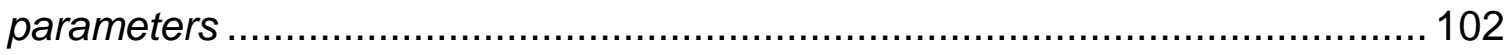

Figure 55. Electronegativity and differences between elements..................... 106

Figure 56. Optimized mixing parameters................................................... 110

Figure 57. The multi-twinned perpendicular nanowire ................................. 111

Figure 58. The difference between the parallel (left) and perpendicular (right) wires

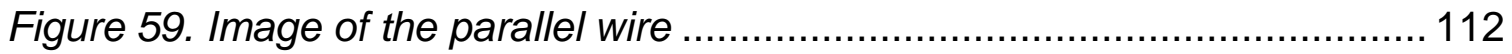

Figure 60. The perpendicular twin wire was lattice optimized......................... 112

Figure 61. Hydrogen passivated multi-twin wire ......................................... 113

Figure 62. Locations of boron substitution and interstitial placement. .............. 114 
Figure 63. Results of doped multi-twin wire ................................................ 115

Figure 64. Substitution of boron near the surface ........................................ 115

Figure 65. EDOS plot of lowest energy substitutional and interstitial cases in twin-

wire. 116

Figure 66. Electronic density of states for the undoped MTSNW 118

Figure 67. Boron placed outside and phosphorus near the center of the core and enlargement. 119

Figure 68. Electronic density of states for the Bout doped MTSNW 119

Figure 69. Boron on the inside and phosphorus on the outside 120

Figure 70. Electronic density of states for the Pout doped MTSNW. 120

Figure 71. The higher energy state is boron in phosphorus out. 121

Figure 72. HOMO-LUMO shift due to Bout/Pout swap .................................. 121

Figure 73. The pair distribution function. ................................................... 122

Figure 74. Angle distribution function for the MTSNW................................. 123

Figure 75. Surface reconstruction in the MTSNW ...................................... 123 


\section{INTRODUCTION}

In 2006 the Condensed Matter Theory (CMT) group at the University of Louisville published a body of work detailing a powerful semi-empirical approach to electronic structure problems ${ }^{1}$. This approach was uniquely different than previous semi-empirical approaches in that it included self-consistency, environment dependence and multicenter interactions. This approach has been coined by the CMT group as The Self-Consistent Environment Dependent Linear Combination of Atomic Orbital Method (SCED-LCAO). The difference between SCED-LCAO method and previous methods is that when one thinks of semiempirical methods you may think of the Hückel ${ }^{2}$, the extended Hückel ${ }^{3}$ methods or you may think of newer semi-empirical methods such as $\mathrm{AM} 1^{4}$ and other $\mathrm{NDDO}^{5}$ methods. These methods neglect large parts of the many-body Hamiltonian - sacrificing accuracy in both the older and newer approaches. The CMT's approach accounts for all sections of the many-body Hamiltonian as is the case for ab-initio methods. The major difference between the SCED-LCAO method and most ab-initio methods is the complex and expensive integrals that arise in ab-initio methods are approximated with phenomenological functions cleverly modeled against a database of information. In this sense the SCEDLCAO method of the CMT group trades a very difficult (basically intractable) 
variational problem from density functional theory/coupled cluster theory for a database parameter optimization problem that is database and model driven.

Systematic improvements of an electronic structure calculation may be possible with extremely costly methods such as configuration interaction $(\mathrm{Cl})$, but such methods are only able to model a few number of particles, currently less than 50 on a large computer. Density functional theory (DFT) has no systematic way of improving quality. One can only enlarge the basis set, trying different models for the exchange-correlation and hope for the best compared to a more accurate model. If modeling is possible, DFT may take days or longer to model 100 atoms with a reasonably large sized parallel computer. When comparing any electronic structure calculation result to a more accurate model such as $\mathrm{Cl}$, you are limited in the size of the system that one can compare. For example, if comparing your DFT results to $\mathrm{Cl}$, you may only be able to test up to approximately 20 atoms before you cannot run the $\mathrm{Cl}$ simulation anymore. In this sense we give up a lot by relying on DFT alone, and in the end are limited to less than 100 atom simulations. This is far less than is needed for the accurate simulation of small nanostructures and surface interactions, which require hundreds if not thousands or tens of thousands of atoms to simulate properly.

Highly stripped down electronic structure methods could be used, but those methods sacrifice an unacceptable amount of accuracy creating unreliable results. Traditional tight binding methods could be used alternatively, however they generally only work for one single system and can fail completely for a slightly different system causing a need for recalibration to a database which is 
highly time consuming and human effort intensive. Given this, the CMT group at University of Louisville has addressed all of these dilemmas with the SCEDLCAO method. The SCED-LCAO method is far superior to older non-orthogonal tight binding methods, yet far less costly than DFT methods. Not just in terms of speed, but also in terms of memory usage, numerical stability and human effort. This makes the method quite attractive to researchers examining larger nanostructures and biological molecules.

To demonstrate the effectiveness of the SCED-LCAO method, the method was applied to the difficult problem of binding energy versus relative atomic volume of different phases of bulk silicon, the stable structure of an intermediatesize $\mathrm{Si}_{71}$ cluster, (iii) the reconstruction of $\mathrm{Si}(100)$ surface, and many other systems involving silicon and carbon ${ }^{1,6-9}$. The ability to model effectively such a large number of systems speaks to the core goal of the SCED-LCAO method; that it is transferable, reliable and has ease of calculation. In every case listed above, the same Hamiltonian construction methodology was used for silicon and carbon. When comparing the SCED-LCAO method to older tight binding methods, there was a vast improvement with the SCED-LCAO method. The speed in which these calculations were performed is on the order of minutes, not days. One cannot help but be amazed at this feat considering that the Hamiltonian for silicon and carbon are functionally the same. This leads us to the question of: Can we model every $\mathrm{S}$ and $\mathrm{P}$ block element with this same general Hamiltonian? Carbon and silicon both occur in the same column on the periodic table. What if we go to the left and try to model boron? What if we go to the right 
and try to model phosphorus? It will be demonstrated that the answer is an astounding yes with minor modifications for boron and phosphorus.

It has long been assumed that one cannot model boron compounds with a semi-empirical method very effectively for several reasons ${ }^{10}$. It was thought that the complicated nature of the 3 center bond, with deficiency of the un-occupied $p$ orbitals $^{11}$, and the ability of boron to bond in so many different energetically

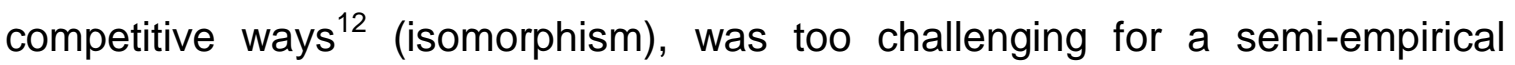
method of any kind. Some of the first attempts to model boron with the extended Hückel method involved modeling boron hydrides ${ }^{13}$ and although that method has some limited accuracy, it is insufficient for any type of electronic structure calculation that would remotely compete with density functional theory. DFT also has problems with modeling boron if the basis set is small and one does not use a hybrid functional ${ }^{14}$. The SCED-LCAO method is not only able model homogeneous boron clusters, sheets, and extended structures accurately; it can also model phosphorus structures. The key is careful construction of the Hamiltonian, the database, and a sound methodology for determination of quality of the SCED-LCAO parameters. If constructed carefully the SCED-LCAO Hamiltonian utilized for boron and phosphorus is very competitive with DFT.

The interest in boron nanostructures over the last 6 years has grown dramatically, the maximum size of these structures of interest are approximately 100 atoms. The reason why is because approximately 100 atoms represents the computational limit DFT can model, given a reasonable amount of time, and computational resources with modern machines. In the near future this will 
increase, but not to the point where it will be possible to effectively model thousands of atoms with DFT reliably.

In contrast, it is very easy for the SCED-LCAO method to model boron on the order of 1,000 atoms with direct diagonalization techniques, and many thousands of atoms with an order $\mathrm{N}$ method. Details of the order $\mathrm{N}$ method and philosophy of the SCED-LCAO method with selected applications to silicon are explained in previous works by the CMT group at the University of Louisville ${ }^{1,15,16}$. This dissertation will explore the alteration of the Hamiltonian to improve the accuracy of the boron Hamiltonian along with the heterogeneous case of mixing multiple different elements together. I will outline in detail the beginning to end methodology of how the boron and phosphorus parameters were found, and used to simulate the behavior of (i) large boron clusters, sheets and extended structures (ii) intermediate sized phosphorus structures (iii) large silicon twin wires that have been doped with boron and phosphorus either individually or simultaneously. 


\title{
CHAPTER I: DESIGN OF A MODEL HAMILTONIAN
}

\author{
AND OVERLAP
}

\subsection{GENERAL STRUCTURE OF THE HAMILTONIAN}

\subsubsection{Hamiltonian matrix elements}

To review, the original Hamiltonian was published in $2006^{1}$ and the general full many-body Hamiltonian can be stated as:

$$
H=-\sum_{l} \frac{\hbar^{2}}{2 m} \nabla_{l}^{2}+\sum_{l, i} v\left(\vec{r}_{l}-\vec{R}_{i}\right)+\sum_{l, l^{\prime}} \frac{e^{2}}{4 \pi \varepsilon_{0} r_{l l^{\prime}}}+\sum_{i, j} \frac{Z_{i} Z_{j} e^{2}}{4 \pi \varepsilon_{0} R_{i j}}
$$

It is critical that we start with the full many-body Hamiltonian, and thus demonstrate that no interaction is left unaccounted. The summation over $l, l^{\prime}$ and $i, j$ runs over all of the valence electrons and ions respectively, where $r_{l l^{\prime}}$ represents the distance between a given electron $l$ and another electron $l$, and $R_{i j}$ the inter-ionic distance between an ion at $R_{i}$ and another ion at $R_{j}$. The potential energy term acts between any electron at $r_{l}$ and ion at $R_{i}$. The number of valence electrons associated with the ion at site at $R_{i}$ is $Z_{i}$. The full many-body formulation is intractable for even small systems. Ultimately the goal is to simulate hundreds if not thousands of atoms. Due to this, the one electron approximation is utilized. In this formulation the electron is seen as moving in an 
external field Veff of all other electrons, all effective interactions are included in this potential:

$$
\hat{H}=-\frac{\hbar^{2}}{2 m} \nabla^{2}+\sum_{i} v\left(\vec{r}-\vec{R}_{i}\right)+V e f f
$$

The Hamiltonian is partitioned into the "intra" and "inter" cases. "Intra" meaning interactions between a valence electron and an electron or ion associated with the same site, and "inter" meaning interactions between a valence electron and an electron or ion associated with a different site. From equation 1.2:

$$
\begin{aligned}
& \sum_{i} v\left(\vec{r}-\vec{R}_{i}\right)=v_{\text {intra }}(\vec{r}-\vec{R})+\sum_{k} v_{\text {inter }}\left(\vec{r}-\vec{R}_{k}\right) \\
& \mathrm{Veff}=\operatorname{Veff}_{\text {intra }}+\mathrm{Veff}_{\text {inter }}
\end{aligned}
$$

The Hamiltonian becomes:

$$
\begin{aligned}
& \hat{H}=-\frac{\hbar^{2}}{2 m} \nabla^{2}+\left\{v_{\text {intra }}(\vec{r}-\vec{R})+\text { Veff }_{\text {intra }}\right\} \\
& +\left\{\sum_{k} v_{\text {inter }}\left(\vec{r}-\vec{R}_{k}\right)+\mathrm{Veff}_{\text {inter }}\right\}
\end{aligned}
$$

The Hamiltonian matrix elements are: 


$$
\begin{aligned}
& H_{i \alpha, j \beta}=\left\langle\varphi_{i \alpha}|\hat{H}| \varphi_{j \beta}\right\rangle \\
& =\left\langle\varphi_{i \alpha}\right|-\frac{\hbar^{2}}{2 m} \nabla^{2}+\left\{v_{\text {intra }}(\vec{r}-\vec{R})+\mathrm{Veff}_{\text {intra }}\right\}+ \\
& \left\{\sum_{k} v_{\text {inter }}\left(\vec{r}-\vec{R}_{k}\right)+\operatorname{Veff}_{\text {inter }}\right\}\left|\varphi_{j \beta}\right\rangle
\end{aligned}
$$

Every term in the Hamilton is then divided into onsite and offsite pairs. Keep in mind that $S_{i \alpha, j \beta}=\left\langle\varphi_{i \alpha} \mid \varphi_{j \beta}\right\rangle$. The matrix elements are evaluated term by term. Starting with the kinetic energy plus the energy of interaction with its own ionic core:

$$
\begin{aligned}
& \left\langle\varphi_{i \alpha}\left|\left[-\frac{\hbar^{2}}{2 m} \nabla^{2}+v_{\text {intra }}(\vec{r}-\vec{R})\right]\right| \varphi_{j \beta}\right\rangle= \\
& \left\langle\varphi_{i \alpha}\left|\left[-\frac{\hbar^{2}}{2 m} \frac{\left(\nabla_{i}^{2}+\nabla_{j}^{2}\right)}{2}+\frac{v_{\text {intra }}\left(\vec{r}-\vec{R}_{i}\right)+v_{\text {intra }}\left(\vec{r}-\vec{R}_{j}\right)}{2}\right]\right| \varphi_{j \beta}\right\rangle= \\
& {\left[\frac{\varepsilon_{i \alpha}^{0}+\varepsilon_{j \beta}^{0}}{2}\right]\left\langle\varphi_{i \alpha} \mid \varphi_{j \beta}\right\rangle=\left[\frac{\varepsilon_{i \alpha}^{0}+\varepsilon_{j \beta}^{0}}{2}\right] S_{i \alpha, j \beta}}
\end{aligned}
$$

The energy of interaction of the onsite electron with other electrons associated with the same ionic site is:

$$
\begin{aligned}
& \left\langle\varphi_{i \alpha}\left|\operatorname{Veff}_{\text {intra }}\right| \varphi_{j \beta}\right\rangle= \\
& \frac{\left[u_{i \alpha}^{\text {intra }}+u_{j \beta}^{\text {intra }}\right]}{2}\left\langle\varphi_{i \alpha} \mid \varphi_{j \beta}\right\rangle=\left[\frac{u_{i \alpha}^{\text {intra }}+u_{j \beta}^{\text {intra }}}{2}\right] S_{i \alpha, j \beta}
\end{aligned}
$$

The energy of interaction of the onsite electron with other electrons associated with other ionic sites: 


$$
\begin{aligned}
& \left\langle\varphi_{i \alpha}\left|V e f f_{\text {inter }}\right| \varphi_{j \beta}\right\rangle= \\
& \frac{\left[u_{i \alpha}^{\text {inter }}+u_{j \beta}^{\text {inter }}\right]}{2}\left\langle\varphi_{i \alpha} \mid \varphi_{j \beta}\right\rangle=\left[\frac{u_{i \alpha}^{\text {inter }}+u_{j \beta}^{\text {inter }}}{2}\right] S_{i \alpha, j \beta}
\end{aligned}
$$

Lastly, the energy of interaction between the onsite electron and the ions at the other sites is:

$$
\begin{aligned}
& \left\langle\varphi_{i \alpha}\left|\sum_{k} v_{\text {inter }}\left(\vec{r}-\vec{R}_{k}\right)\right| \varphi_{j \beta}\right\rangle= \\
& \left\langle\varphi_{i \alpha}\left|\frac{1}{2}\left[\sum_{k} v_{\text {inter }}\left(\vec{r}-\vec{R}_{i k}\right)+\sum_{k} v_{\text {inter }}\left(\vec{r}-\vec{R}_{j k}\right)\right]\right| \varphi_{j \beta}\right\rangle= \\
& \frac{\left[v_{i \alpha}^{\text {inter }}+v_{j \beta}^{\text {inter }}\right]}{2}\left\langle\varphi_{i \alpha} \mid \varphi_{j \beta}\right\rangle=\left[\frac{v_{i \alpha}^{\text {inter }}+v_{j \beta}^{\text {inter }}}{2}\right] S_{i \alpha, j \beta}
\end{aligned}
$$

Collecting terms we obtain a final expression for our matrix element:

$$
H_{i \alpha, j \beta}=\frac{1}{2}\left(\begin{array}{l}
\varepsilon_{i \alpha}^{0}+\varepsilon_{j \beta}^{0}+u_{i \alpha}^{\text {intra }}+u_{j \beta}^{\text {intra }}+ \\
u_{i \alpha}^{\text {inter }}+u_{j \beta}^{\text {inter }}+v_{i \alpha}^{\text {inter }}+v_{j \beta}^{\text {inter }}
\end{array}\right) S_{i \alpha, j \beta}
$$

When $i \alpha=j \beta$ we have the onsite elements of the Hamiltonian and $S_{i \alpha, j \beta}$ are equal to one, reducing the onsite expression to $H_{i \alpha, i \alpha}$, so our final Hamiltonian matrix elements are:

$$
\begin{aligned}
& \text { onsite: } H_{i \alpha, i \alpha}=\varepsilon_{i \alpha}^{0}+u_{i \alpha}^{\text {intra }}+u_{i \alpha}^{\text {inter }}+v_{i \alpha}^{\text {inter }} \\
& \text { offsite: } H_{i \alpha, j \beta}=\frac{1}{2}\left(\begin{array}{c}
\varepsilon_{i \alpha}^{0}+\varepsilon_{j \beta}^{0}+u_{i \alpha}^{\text {intra }}+u_{j \beta}^{\text {intra }}+ \\
u_{i \alpha}^{\text {inter }}+u_{j \beta}^{\text {inter }}+v_{i \alpha}^{\text {inter }}+v_{j \beta}^{\text {inter }}
\end{array}\right) S_{i \alpha, j \beta}
\end{aligned}
$$

The only approximation made at this point is that we are working within a one electron Hamiltonian; these matrix elements reflect complex integrals that include all interactions of the full many-body Hamiltonian. The evaluation of these 
integrals is a notoriously unwieldy and cannot be solved in general for larger systems. We circumvent all cumbersome integrations by representing each integral with a single, very simple yet appropriate function or set of functions. The function(s) are cleverly chosen based upon strong physical assumptions. This approach has several distinct advantages. All terms have physical significance with a small number of parameters. It was not by design, that the number of parameters in the Hamiltonian is small. It was found that increasing the number of parameters and functions in the Hamiltonian did not improve its quality, and the fitting time increased greatly and unnecessarily. The number of parameters that were used is not only optimal, but sufficient to represent the physics and computationally preferable. The accuracy of the Hamiltonian is quite remarkable and that will be demonstrated in the case of boron, an extremely difficult element to model. Parameterized functions were created for these matrix elements and reflect the physical behavior of the system.

\subsubsection{Interaction Terms and modeling}

To gain the most accuracy and retain as much flexibility as possible in the SCED-LCAO model, the physics of the system is exploited as opposed to simply increasing the number of parameters/functions or using arbitrary functions with no physical basis. This is the true strength of this method. Only through carefully modeling the individual interactions can a reliable Hamiltonian (that will work in many different scenarios) be obtained. This was a critical decision made early in the method development, and one that distinguishes this approach from older tight binding methods. This approach allows for very rapid and accurate 
calculations based upon the need for minimal computation in a molecular modeling scheme.

Standard tight binding techniques tend to be unreliable when applied to different systems across many different scenarios (unreliable, non-transferable), because they are not physically based models. The approach used in the SCEDLCAO method increases transferability and thus reliability with the careful additions of multicenter interactions, self-consistency, and a delocalization function (this function was added for boron and phosphorus Hamiltonians), and a sound physically modeled framework. We have also extended the method beyond homogenous systems (i.e. systems that contain only one type of atom such as silicon). I will demonstrate that the Hamiltonian is so flexible, that heterogeneous systems that contain many different kinds of atoms can be modeled.

\subsubsection{Onsite electron and onsite ion interaction energy, plus the electron kinetic energy}

The approximation for the onsite electron and onsite ion interaction energy, plus the electron kinetic energy reflects three independent pieces of phenomena. We use a constant term $\varepsilon_{i \alpha}$ which may be construed as the energy of the $\alpha$ orbital of the isolated atom at i. See Figure 1. This term is pinned (fixed) to single atom values for the valence level ${ }^{17,18}$ and generally it is not allowed to vary much. $Z_{i}$ represents the ionic charge for a given atomic species, and is also fixed. The term $Z_{i} U_{i}$ is the product of the ionic charge where $U$ is an effective 
Hubbard ${ }^{19}$ like approximation to describe the intra-atomic electron interactions. Tables $^{18}$ of atomic data are initially used for $\varepsilon_{i \alpha}$ and $U$, but $U$ is allowed to vary slightly according to our fitting process which will be described later. These tables are available in Walter Harrison's book or the paper cited above. This is justified by the fact that $U$ was originally calculated using the assumption of the isolated atomic case. It does not reflect the fact that the intra-atomic interaction will be altered by the correlation effects of the environment once we have a very large number atoms in our system, but, $U$ is not allowed to vary drastically from the isolated atomic case, so we soft constrain this parameter in the code.

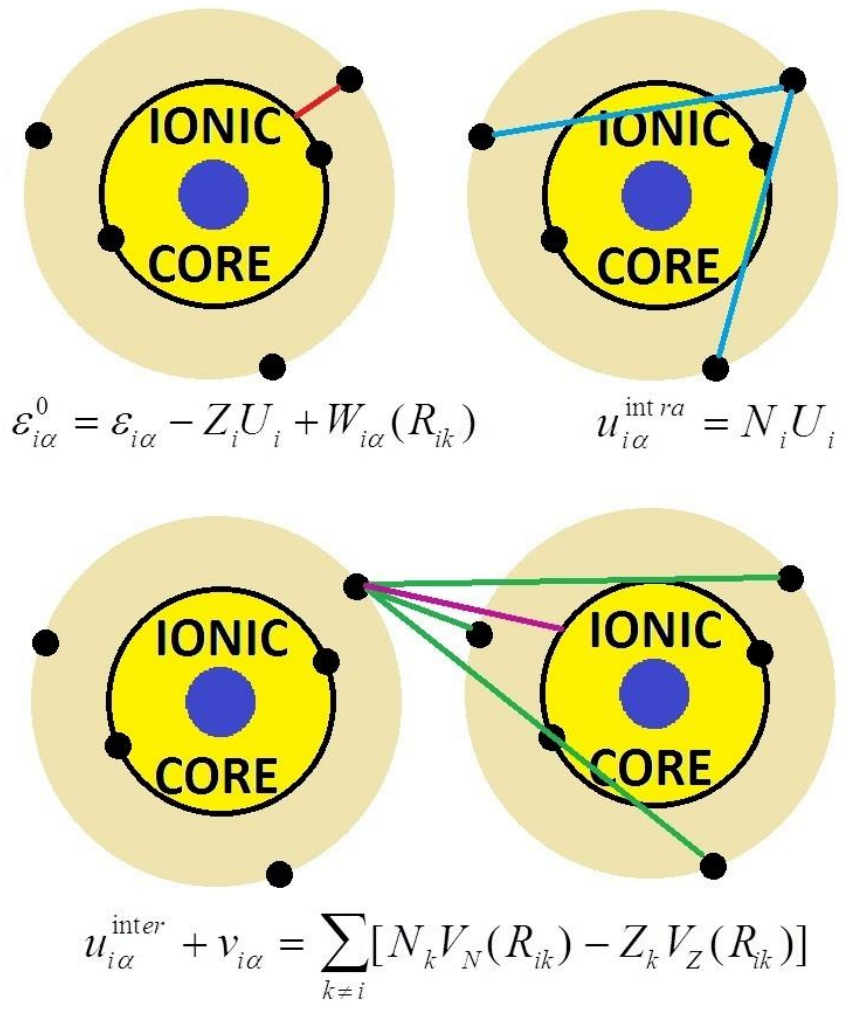

Figure 1. Onsite model approximations 
Onsite and offsite approximations for $\varepsilon_{i \alpha}^{0}$ are in equation 1.12:

$$
\begin{aligned}
& \text { onsite : } \varepsilon_{i \alpha}^{0}=\varepsilon_{i \alpha}+W_{i \alpha}\left(R_{i k}\right)-Z_{i} U_{i} \\
& \text { offsite }: \varepsilon_{i \alpha}^{0}=\left[\varepsilon_{i \alpha}^{\prime}+\sum_{k \neq i} W_{i \alpha}\left(R_{i k}\right)\right] K\left(R_{i j}\right)-Z U_{i} \\
& \text { offsite : } \varepsilon_{j \beta}^{0}=\left[\varepsilon_{j \beta}^{\prime}+\sum_{k \neq j} W_{j \beta}\left(R_{j k}\right)\right] K\left(R_{i j}\right)-Z U_{j}
\end{aligned}
$$

For a P block system (e.g. boron) we will have two terms $\varepsilon_{i s}$ and $\varepsilon_{i p}$. One could imagine if this technique was explored using a D-block system it would also include $\varepsilon_{i d}$. The parameter $\varepsilon_{i \alpha}$ is fixed in the SCED-LCAO model for the onsite case. The offsite case roughly follows the Wolfsberg-Helmholtz ${ }^{20}$ relation from extended Hückel theory ${ }^{21}$ with $K\left(R_{i j}\right)$ being a function of separation distance (not a constant ${ }^{20}$ ) following Hoffman's methodology ${ }^{21}$. To justify this, the realization that as the separation distance is decreased, $K\left(R_{i j}\right)$ must asymptotically approach a constant value of 1 as $R_{i j}$ get's small, but 1 for the whole range of $K\left(R_{i j}\right)$ would give very poor performance as evidenced by the work of Lipscomb and Hoffman where $K\left(R_{i j}\right)$ was pinned (fixed) at 1 for boron hydrides ${ }^{13}$. Also note that the value of 1.75 used by Hoffman across the whole range of interaction performs poorly, but does provide a good initial guess. The model we incorporated for $K\left(R_{i j}\right)$ is a weak exponential model (fairly flat over the range of interest), which appeared to work out well over the range of separation considered: 


$$
K\left(R_{i j}\right)=e^{\alpha_{i j, K} R_{i j}}
$$

The free parameter $\alpha_{i j, \mathrm{~K}}$ is small accordingly, as would be expected.

The free parameter $\varepsilon_{i \alpha}^{\prime}$ is being used for the offsite case as opposed to $\varepsilon_{i \alpha}$ for the onsite case. Critical flexibility in the SCED-LCAO model is lost if these terms are allowed to be equivalent. The reason they must be different is due the shift in energy from the environment of the offsite case vs. the onsite cases. This effectively shifts the energy for the offsite case, this phenomenon is exacerbated as the system size increases to larger and larger molecules, the types discussed in this project. Also, if the $W_{i \alpha}\left(R_{i k}\right)$ functions are allowed to be different in the onsite and offsite cases, a large number of free parameters are introduced into the model, and this can greatly increase computational time. $\varepsilon_{i \alpha}^{\prime}$ is allowed to deviate away from $\varepsilon_{i \alpha}$ a bit, thus minimizing the number of free parameters to be optimized, we will not optimize both.

The final phenomenological function included in the evaluation of $\varepsilon_{i \alpha}^{0}$ is the $W_{i \alpha}\left(R_{i k}\right)$ function; this represents a critical change to the earlier SCED-LCAO model ${ }^{1}$. When trying to create a set of parameters for hydrogen, it was noted by another doctoral student, Mr. Harrison Simrall, that although we can use $-13.6 \mathrm{eV}$ for the value of $\varepsilon_{i s}$ and $\mathrm{oeV}$ for the value of $\varepsilon_{i p}$ it is far more effective to raise the energy up to $-4.0 \mathrm{eV}$ for $\varepsilon_{i s}$. This result was puzzling because it seemed unphysical. The explanation for this difference in energy is justified because our 
parameters are fit to decent sized molecules of many atoms (and not to single atom systems). The single atom result for the valence levels has to be compensated (increased) to allow for the case when multiple atoms are brought near each other, as opposed to the single case. The addition of the $W_{i \alpha}\left(R_{i k}\right)$ function compensates for the multiple atom dilemma concerning this energy shift. $W_{i \alpha}\left(R_{i k}\right)$ must have the property that at infinity or near the cutoff, the function must vanish, but at shorter distance the function must be finite and alter the single atom result for the valence level term $\varepsilon_{i \alpha}$ accordingly. This will be demonstrated to greatly improve the quality of the boron Hamiltonian. In general the simplest function with the least number of parameters (that retains the above behavior) is a decaying exponential with two free parameters to be optimized

during a fitting process as shown in equation 1.14. Although this function is not strong for boron, it greatly improved the ability for the Hamiltonian to model boron. It is expected that for an S block element like hydrogen, this function would be absolutely critical.

$$
W_{i \alpha}\left(R_{i j}\right)=W_{i \alpha}^{0} e^{-\alpha_{i \alpha, W} R_{i j}}
$$

\subsubsection{Onsite electron and onsite electron interaction energy}

The onsite electron and onsite electron interaction energy is very simply modeled with the product of the variable $N_{i}$ and the Hubbard term. 


$$
\begin{aligned}
& \text { on }: u_{i \alpha}^{\text {intra }}=N_{i} U_{i} \\
& \text { off }: u_{i \alpha}^{\text {int } r a}=N_{i} U_{i} \\
& \text { off }: u_{j \beta}^{\text {int } r a}=N_{j} U_{j}
\end{aligned}
$$

This deceivingly simple product will be the first key to charge redistribution and self-consistency in this formulation. The value $N_{i}$ will be allowed to vary until self-consistency is reached.

\subsubsection{Onsite electron and offsite ion interaction energy, plus, onsite electron and offsite electron interaction energy}

The onsite electron and offsite ion interaction energy, plus, onsite electron and offsite electron interaction energy is a complex formulation detailed in two

previous works ${ }^{1,15}$. I refer the reader to these works. The formulation for this body of work from the previous models has not been changed. To review, this term includes environment-dependent multi-center (three-center explicitly and fourcenter implicitly) interactions. By 3 centers implicitly we see that centers $i, j, k$ are involved explicitly. The involvement of 4 centers appears when we note that $N_{k}$ is calculated from the eigenvalues of one set of centers then mixed with centers located at $i, j, k$.

$$
N_{k}=\sum_{\alpha, \lambda}\left(c_{k \alpha}^{\lambda}\right)^{2}+\sum_{\alpha, l \beta, \lambda(l \neq k)} c_{k \alpha}^{\lambda} c_{l \beta}^{\lambda} S_{l \beta, k \alpha}
$$

Our onsite and offsite terms are thus: 


$$
\begin{aligned}
& \text { on: } u_{i \alpha}^{\text {inter }}+v_{i \alpha}=\sum_{k \neq i}\left[N_{k} V_{N}\left(R_{i k}\right)-Z_{k} V_{Z}\left(R_{i k}\right)\right] \\
& \text { off }: u_{i \alpha}^{\text {inter }}+v_{i \alpha}^{\text {int } e r}=\sum_{k \neq i}\left(N_{k} V_{N}\left(R_{i k}\right)-Z_{k} V_{Z}\left(R_{i k}\right)\right) \\
& \text { off }: u_{j \beta}^{\text {inter }}+v_{j \beta}^{\text {inter }}=\sum_{k \neq j}\left(N_{k} V_{N}\left(R_{j k}\right)-Z_{k} V_{Z}\left(R_{j k}\right)\right)
\end{aligned}
$$

\subsubsection{Assembling the Hamiltonian}

The full Hamiltonian may now be assembled into its final form. The onsite elements are:

$$
\begin{aligned}
& \text { on: } H_{i \alpha, i \alpha}=\left\{\varepsilon_{i \alpha}^{0}\right\}+\left\{u_{i \alpha}^{\text {intra }}\right\}+\left\{u_{i \alpha}^{\text {inter }}+v_{i \alpha}^{\text {inter }}\right\} \\
& H_{i \alpha, i \alpha}=\left\{\varepsilon_{i \alpha}+W_{i \alpha}\left(R_{i k}\right)-Z_{i} U_{i}\right\}+\left\{N_{i} U_{i}\right\}+ \\
& \left\{\sum_{k \neq i}\left[N_{k} V_{N}\left(R_{i k}\right)-Z_{k} V_{Z}\left(R_{i k}\right)\right]\right\} \\
& =\varepsilon_{i \alpha}+W_{i \alpha}\left(R_{i k}\right)+\left(N_{i}-Z_{i}\right) U_{i}+ \\
& \sum_{k \neq i}\left[N_{k} V_{N}\left(R_{i k}\right)-Z_{k} V_{Z}\left(R_{i k}\right)\right]
\end{aligned}
$$

The offsite elements are:

$$
\begin{aligned}
& \text { off } \left.: H_{i \alpha, j \beta}=\frac{1}{2}\left(\begin{array}{l}
\left\{\varepsilon_{i \alpha}^{0}+\varepsilon_{j \beta}^{0}\right\}+\left\{u_{i \alpha}^{\text {intra }}+u_{j \beta}^{\text {intra }}\right\} \\
\left\{u_{i \alpha}^{\text {inter }}+u_{j \beta}^{\text {inter }}+v_{i \alpha}^{\text {inter }}+v_{j \beta}^{\text {inter }}\right.
\end{array}\right\}\right) S_{i \alpha, j \beta} \\
& = \\
& =\frac{1}{2}\left(\begin{array}{l}
{\left[\varepsilon_{i \alpha}^{\prime}+\sum_{k \neq i} W_{i \alpha}\left(R_{i k}\right)+\varepsilon_{j \beta}^{\prime}+\sum_{k \neq j} W_{j \beta}\left(R_{j k}\right)\right] K\left(R_{i j}\right)} \\
+\left(N_{i}-Z_{i}\right) U_{i}+\left(N_{j}-Z_{j}\right) U_{j} \\
+\sum_{k \neq i}\left(N_{k} V_{N}\left(R_{i k}\right)-Z_{k} V_{Z}\left(R_{i k}\right)\right)+ \\
\sum_{k \neq j}\left(N_{k} V_{N}\left(R_{j k}\right)-Z_{k} V_{Z}\left(R_{j k}\right)\right)
\end{array}\right) S_{i \alpha, j \beta}
\end{aligned}
$$


The phenomenological functions are:

$$
\begin{aligned}
& W_{\alpha}\left(R_{i k}\right)=W_{i \alpha}^{0} e^{-\alpha_{i \alpha, W} R_{i j}} \\
& K\left(R_{i j}\right)=K_{i j}^{0} e^{\alpha_{i j, K} R_{i j}} \\
& V_{Z}\left(R_{i k}\right)=\frac{E_{0}}{R_{i k}}\left\{1-\left(1+B_{Z} R_{i k}\right) e^{-\alpha_{Z} R_{i k}}\right\} \\
& \Delta V_{N}\left(R_{i k}\right)=\left(A_{N}+B_{N} R_{i k}\right) \frac{\left[1+e^{-\alpha_{N} d_{N}}\right]}{\left[1+e^{-\alpha_{N}\left(d_{N}-R_{i k}\right)}\right]} \\
& V_{N}\left(R_{i k}\right)=V_{Z}\left(R_{i k}\right)+\Delta V_{N}\left(R_{i k}\right)
\end{aligned}
$$

Note that the parameters in the above expressions pertain to the $i^{\text {th }}$ element and this formulation is in general a homogeneous, all atoms are the same element. In chapter 5 the heterogeneous formulation will be introduced for dissimilar elements. When using different elemental types, such as phosphorus and boron, there will be mixing parameters also when the function is not site specific. This is only required for heterogeneous systems.

It is to be noted that if referencing $W_{i \alpha}\left(R_{i j}\right)=W_{i \alpha}^{0} e^{-\alpha_{i \alpha, W} R_{i j}}$ for the $i=3$ and $k=7$ case that $W_{3 \alpha}\left(R_{37}\right)=W_{3 \alpha}^{0} e^{-\alpha_{3 \alpha, W} R_{37}}$ and the $W_{3 \alpha}^{0}$ and $\alpha_{3 \alpha, W}$ parameters will be used for the element that belongs to the $i=3$ site, not the $k=7$ site. Those parameters would be the same for the homogeneous system, but different for the heterogeneous system. Note that the $\alpha$ in the subscript refers to $\mathrm{s}$ or $\mathrm{p}$, for example $W_{3 s}\left(R_{37}\right)=W_{3 s}^{0} e^{-\alpha_{3 s, W} R_{37}}$, whereas $\alpha_{i \alpha, W}$ is the exponentially related parameter for $W_{i \alpha}\left(R_{i j}\right)$. 


\subsubsection{Shape of the Hamiltonian}

The Hamiltonian is must be square and Hermitian. For every site there is an associated block, for example the $i=1, j=6$ has an associated block. There is not an explicit basis set; a phenomenological overlap will be used. But, we will be working within a minimal basis set formulation, so the block size for our Hamiltonian at any given site is dictated by the minimum number of basis functions required to represent all of the electrons on each atom.

In the case of boron (in the $\mathrm{P}$ block) we will have a $\mathrm{sp}$ system $\left\{s, p_{x}, p_{y}, p_{z}\right\}$ a $4 \times 4$ block for each element, or 16 sub-elements is used. If we had a D block element such as iron, we would have a much larger block (each block would be $9 \times 9$, or 81 sub-elements). So the Hamiltonian size is $\boldsymbol{m n} \times \boldsymbol{m n}$ where $\boldsymbol{m}$ is the size of the minimal basis set, and $\boldsymbol{n}$ is the number of atoms in the system. We will need to evaluate ${ }_{m n}$ onsite Hamiltonian elements and $m n(m n-1)$ offsite Hamiltonian elements in general. Keep in mind this will be done during each self-consistency loop. If a system has a poor initial guess, it could take an extremely long time to reach self-consistency, or the algorithm could fail completely. Turning off self-consistency or relaxing the stopping criteria can also be a problem. Having a good initial set of parameters for your Hamiltonian is critical. A cutoff was implemented for the Hamiltonian where interactions beyond a certain distance were defined as zero. This introduces many zeros into our Hamiltonian making the solution of the 
generalized eigenvalue problem much easier. The general Hamiltonian is as such:

$$
H_{i \alpha, j \beta}=\left[\begin{array}{cccc}
1,1 & 1,2 & \ldots & 1, n \\
2,1 & 2,2 & \ldots & 2, n \\
\ldots & \ldots & \ldots & \ldots \\
n, 1 & n, 2 & \ldots & n, n
\end{array}\right]
$$

With blocks as such:

$$
\begin{aligned}
& i, i=\begin{array}{cccc}
H_{s s \sigma}^{i, i} & 0 & 0 & H_{s p \sigma}^{i, i} \\
0 & H_{p p \pi}^{i, i} & 0 & 0 \\
0 & 0 & H_{p p \pi}^{i, i} & 0 \\
H_{p s \sigma}^{i, i} & 0 & 0 & H_{p p \sigma}^{i, i} \\
\hline
\end{array} \\
& \underset{i \neq j}{i, j}=\begin{array}{cccc}
H_{s s}^{i, j} & H_{s s_{x}}^{i, j} & H_{s p_{y}}^{i, j} & H_{s p_{z}}^{i, j} \\
H_{p_{p_{x}} s}^{i, j} & H_{p_{x} p_{x}}^{i, j} & H_{p_{x} p_{y}}^{i, j} & H_{p_{x} p_{z}}^{i, j} \\
H_{p_{y} s}^{i, j} & H_{p_{y} p_{x}}^{i, j} & H_{p_{y} p_{y}}^{i, j} & H_{p_{y} p_{z}}^{i, j} \\
H_{p_{z} s}^{i, j} & H_{p_{z} p_{x}}^{i, j} & H_{p_{z} p_{y}}^{i, j} & H_{p_{z} p_{z}}^{i, j} \\
\hline
\end{array}
\end{aligned}
$$

\subsection{GENERAL STRUCTURE OF THE OVERLAP AND ENERGY}

\subsubsection{Limit Requirements of the Phenomenological functions}

We will be working with overlap functions instead of wave functions. The overlap function from an ab-initio calculation must be similar in shape to the phenomenological overlap function used in SCED-LCAO. The linear combination of atomic orbitals represents a localized representation of a generalized basis 
set. We will be working in real space and we will never be working in $\mathrm{k}$ space for our molecular mechanics routines. This has some distinct advantages when analyzing clusters and small nanostructures as opposed to calculations involving large extended structures like an infinite slab, an infinite crystal, or an infinite surface. In this sense, the goal of this body of work is concerned with finite structures, but we do not exclude infinite extended structures on the contrary they are very well modeled. The database we generate will include plane wave information in order to fit electronic structure information, but we will not be using plane waves in the molecular mechanics routines. ${ }^{22-27}$

All overlap functions must vanish as the separation distance nears infinity. As the separation distance nears zero all overlaps must near unity or zero, and must never exceed unity. This is ensured by using an exponential decay function modulated by a polynomial. One caveat that can occur, for certain values of parameters, is that the unity condition is not met in the numerical sense. This means we can arrive at a set of parameters that can yield an overlap greater than unity. This turns out to be a bigger problem than one would expect because it is difficult to bound the overlap in a constraint. Whereas we can easily places constraints on parameter values; it is a bit more difficult to place upper and lower bounds on overlap functions. This requirement was manually checked during the parameter acquisition stage for boron to ensure that the unity requirement was met. The number of parameters per overlap is limited to 3 per function. 


\subsubsection{An energy statement}

Now that we have our Hamiltonian the energy can be evaluated in a straight forward manner, and follows from the previous work ${ }^{1}$ and presented below for reference. The band structure energy is:

$$
\begin{aligned}
& H_{i \alpha, j \beta}=H_{i \alpha, j \beta}^{0}+\frac{1}{2}\left\{\left[\left(N_{i}-Z_{i}\right) U_{i}+\left(N_{j}-Z_{j}\right) U_{j}\right]\right. \\
& +\left[\sum_{k \neq i}\left(N_{k} V_{N}\left(R_{i k}\right)-Z_{k} V_{Z}\left(R_{i k}\right)\right)\right. \\
& \left.\left.+\sum_{k \neq j}\left(N_{k} V_{N}\left(R_{j k}\right)-Z_{k} V_{Z}\left(R_{j k}\right)\right)\right]\right\} S_{i \alpha, j \beta}\left(R_{i j}\right) \\
& E_{B S}=\sum_{i \alpha, j \beta} \sum_{\lambda}^{o c c} c_{i \alpha}^{\lambda} c_{j \beta}^{\lambda} H_{j \beta, i \alpha} \\
& E_{B S}=\sum_{i \alpha, j \beta} \sum_{\lambda}^{o c c} c_{i \alpha}^{\lambda} c_{j \beta}^{\lambda}\left\{\begin{array}{l}
H_{i \alpha, j \beta}^{0}+\frac{1}{2}\left\{\left[\left(N_{i}-Z_{i}\right) U_{i}+\left(N_{j}-Z_{j}\right) U_{j}\right]\right. \\
+\left[\sum_{k \neq i}\left(N_{k} V_{N}\left(R_{i k}\right)-Z_{k} V_{Z}\left(R_{i k}\right)\right)\right. \\
\left.\left.+\sum_{k \neq j}\left(N_{k} V_{N}\left(R_{j k}\right)-Z_{k} V_{Z}\left(R_{j k}\right)\right)\right]\right\} S_{i \alpha, j \beta}\left(R_{i j}\right)
\end{array}\right)
\end{aligned}
$$

Reducing:

$$
\begin{aligned}
& =\sum_{i \alpha, j \beta} \sum_{\lambda}^{o c c} c_{i \alpha}^{\lambda} c_{j \beta}^{\lambda} H_{j \beta, i \alpha}^{0} \\
& +\sum_{i \alpha, j \beta} \sum_{\lambda}^{o c c} c_{i \alpha}^{\lambda} c_{j \beta}^{\lambda}\left\{\begin{array}{l}
{\left[\left(N_{i}-Z_{i}\right) U_{i}+\left(N_{j}-Z_{j}\right) U_{j}\right]} \\
+\left[\sum_{k \neq i}\left(N_{k} V_{N}\left(R_{i k}\right)-Z_{k} V_{Z}\left(R_{i k}\right)\right)+\right. \\
\left.\sum_{k \neq j}\left(N_{k} V_{N}\left(R_{j k}\right)-Z_{k} V_{Z}\left(R_{j k}\right)\right)\right]
\end{array}\right\} \frac{1}{2} S_{i \alpha, j \beta}\left(R_{i j}\right)
\end{aligned}
$$




$$
\begin{aligned}
& E_{B S}^{0}=\sum_{i \alpha, j \beta} \sum_{\lambda}^{o c c} c_{i \alpha}^{\lambda} c_{j \beta}^{\lambda} H_{i \alpha, j \beta}^{0} \\
& =E_{B S}^{0}+\sum_{i \alpha, j \beta} \sum_{\lambda}^{o c c} c_{i \alpha}^{\lambda} c_{j \beta}^{\lambda}\left\{\begin{array}{l}
{\left[\left(N_{i}-Z_{i}\right) U_{i}+\left(N_{j}-Z_{j}\right) U_{j}\right]} \\
+\left[\sum_{k \neq i}\left(N_{k} V_{N}\left(R_{i k}\right)-Z_{k} V_{Z}\left(R_{i k}\right)\right)+\right. \\
\left.\sum_{k \neq j}\left(N_{k} V_{N}\left(R_{j k}\right)-Z_{k} V_{Z}\left(R_{j k}\right)\right)\right]
\end{array}\right\} \frac{1}{2} S_{i \alpha, j \beta}\left(R_{i j}\right) \\
& =E_{B S}^{0}+\left\{\begin{array}{l}
\sum_{i \alpha, j \beta} \sum_{\lambda}^{o c c} c_{i \alpha}^{\lambda} c_{j \beta}^{\lambda}\left\{\left[\left(N_{i}-Z_{i}\right) U_{i}+\left(N_{j}-Z_{j}\right) U_{j}\right]\right\} \\
+\sum_{i \alpha, j \beta} \sum_{\lambda}^{o c c} c_{i \alpha}^{\lambda} c_{j \beta}^{\lambda}\left[\sum_{k \neq i}\left(N_{k} V_{N}\left(R_{i k}\right)-Z_{k} V_{Z}\left(R_{i k}\right)\right)\right. \\
\left.+\sum_{k \neq j}\left(N_{k} V_{N}\left(R_{j k}\right)-Z_{k} V_{Z}\left(R_{j k}\right)\right)\right]
\end{array}\right\} \frac{1}{2} S_{i \alpha, j \beta}\left(R_{i j}\right) \\
& =E_{B S}^{0}+\left\{\begin{array}{l}
\sum_{i \alpha, j \beta} \sum_{\lambda}^{o c c} c_{i \alpha}^{\lambda} c_{j \beta}^{\lambda}\left\{\left[\left(N_{i}-Z_{i}\right) U_{i}+\left(N_{j}-Z_{j}\right) U_{j}\right]\right\} \\
+\sum_{i \alpha, j \beta} \sum_{\lambda}^{o c c} c_{i \alpha}^{\lambda} c_{j \beta}^{\lambda}\left[\sum_{k \neq i}\left(N_{k} V_{N}\left(R_{i k}\right)-Z_{k} V_{Z}\left(R_{i k}\right)\right)\right. \\
\left.+\sum_{k \neq j}\left(N_{k} V_{N}\left(R_{j k}\right)-Z_{k} V_{Z}\left(R_{j k}\right)\right)\right]
\end{array}\right\} \frac{1}{2} S_{i \alpha, j \beta}\left(R_{i j}\right) \\
& E_{B S}=E_{B S}^{0}+\sum_{i}\left(N_{i}-Z_{i}\right) U_{i}\left(\sum_{\alpha, \lambda}\left(c_{i \alpha}^{\lambda}\right)^{2}+\sum_{\alpha, j \beta, \lambda} c_{i \alpha}^{\lambda} c_{j \beta}^{\lambda} S_{j \beta, i \alpha}\right) \\
& +\sum_{k \neq i} \sum_{i}\left[N_{k} V_{N}\left(R_{i k}\right)-Z_{k} V_{Z}\left(R_{i k}\right)\right]\left(\sum_{\alpha, \lambda}\left(c_{i \alpha}^{\lambda}\right)^{2}+\sum_{\alpha, j \beta, \lambda} c_{i \alpha}^{\lambda} c_{j \beta}^{\lambda} S_{j \beta, i \alpha}\right) \\
& N_{i}=\sum_{\alpha, \lambda}\left(c_{i \alpha}^{\lambda}\right)^{2}+\sum_{\alpha, j \beta, \lambda(j \neq i)} c_{i \alpha}^{\lambda} c_{j \beta}^{\lambda} S_{j \beta, i \alpha}
\end{aligned}
$$

Listed below, is the band structure energy statement. By adding the $W_{i \alpha}\left(R_{i j}\right)$ term the shape of the energy statement is not altered.

$$
E_{B S}=E_{B S}^{0}+\sum_{i}\left(N_{i}-Z_{i}\right) N_{i} U_{i}+\sum_{i, k(k \neq i)}\left[N_{k} V_{N}\left(R_{i k}\right)-Z_{k} V_{Z}\left(R_{i k}\right)\right] N_{i}
$$


Once we take into account the subtraction due to double counting and ion-ion repulsion we have the final total energy. Note that in this formulation subtraction of the double counting term is quite easy, unlike in the DFT case.

$$
\begin{aligned}
& E_{\text {tot }}=E_{B S}^{0}+\frac{1}{2} \sum_{i}\left(N_{i}-Z_{i}\right)^{2} U_{i}+\frac{1}{2} \sum_{i, k(k \neq i)} N_{i} N_{k} V_{N}\left(R_{i k}\right) \\
& -\sum_{i, k(k \neq i)} N_{i} Z_{k} V_{Z}\left(R_{i k}\right)+\frac{1}{2} \sum_{i, k(k \neq i)} Z_{i} Z_{k} V_{c}\left(R_{i k}\right) \\
& E_{t o t}=E_{B S}^{0}+\frac{1}{2} \sum_{i} \Delta N_{i}^{2} U_{i}+\frac{1}{2} \sum_{i, k(k \neq i)} \Delta N_{i} \Delta N_{k} V_{N}\left(R_{i k}\right) \\
& +\sum_{i, k(k \neq i)} N_{i} Z_{k} \Delta V_{N}+\frac{1}{2} \sum_{i, k(k \neq i)} Z_{i} Z_{k}\left(\Delta V_{C}-\Delta V_{N}\right)
\end{aligned}
$$

Where,

$$
\begin{aligned}
\Delta N_{i} & =N_{i}-Z_{i} \\
\Delta V_{C} & =\frac{E_{0}}{R_{i k}}\left(1+B_{Z} R_{i k}\right) e^{-\alpha_{Z} R_{i k}}
\end{aligned}
$$

The first term is the band structure energy, the second and third terms represent energy shift due to charge fluctuations after self-consistency is reached. The $4^{\text {th }}$ and $5^{\text {th }}$ terms are short range functions and play a significant role, especially in the case of boron when we have potential energy surfaces with such shallow wells. 


\section{CHAPTER II: BORON PARAMETER ACQUISTION}

\subsection{AN INITIAL PARAMETER GUESS}

In chapter one a semi-empirical Hamiltonian and overlap was constructed. From this, an energy statement was derived. If one supplies the type of element (e.g. boron), the initial coordinates and all parameters, the energy of the system can be rapidly calculated. Finding a parameter set that meets all requirements will be the focus of this chapter. There is no unique way to find these parameters, but I will present the systematic methodology used. The ultimate way of knowing if the parameters yield accurate energies for a given system configuration, involves extensive comparison to an experiment if available. Unfortunately, such experimental data is usually not available or is not in a convenient form for comparison. For example, there may be easy access to band gap data, but there may not be accurate crystalline structure for the material or thermodynamic data. For small clusters experimental data may not be available at all. For this reason, at least initially, we rely upon the rapid calibration of our semi-empirical parameters to databases constructed from ab-initio electronic structure methods of varying accuracy. In this sense the database drives the fitting process. But as you will see, the database does not $100 \%$ uniquely determine the final parameter set during this fitting process. 


\subsubsection{Initial Overlap Parameter Estimates and Constraints}

The nice thing about the SCED-LCAO formulation is that all phenomenological functions in the formulation have a physical basis. One could imagine that we could simply choose arbitrary special functions with free parameters, combine them into linear combinations, and start some sort of organized fitting process. This is a very poor approach resulting in extremely large numbers of free parameters and unpredictable physical and poor behavior outside of our fitting database. Plus, as we move around through our fitting space it could take an extremely long period of time for the database fitting process to converge to a better fit.

The functional form we use for the overlap has 3 parameters, and 4 functions for a sp basis, $s s \sigma, s p \sigma, p p \sigma$ and $p p \pi$ for a total of 12 adjustable fitting parameters for boron or any sp system. The whole model including the complete Hamiltonian and overlap contains only 25 parameters for a sp system, so the overlaps alone represent about half of the fitting parameters. This means we would like to get a rapid, somewhat accurate, initial guess with minimum effort. Our final overlap parameters after completing the fitting process should not look extremely different than the overlap curves we may encounter in a DFT simulation or from tight binding studies or any other electronic structure simulation. They will possibly have slightly different nodal minimums/peaks and decay rates may vary slightly, but the general shape will be similar. Keep in mind that we work upon the premise that small changes in any parameter will most likely impart huge changes in calculated quantities, for example the total energy. 
For an initial guess simply getting close in shape will be sufficient. For this reason we could simply fit our phenomenological functions for our overlap to another method as an initial guess, and this is exactly what we do. The general overlap function we use is:

$$
S_{i j, \tau}=\left(A_{\tau}+B_{\tau} R_{i j}\right) \frac{\left[1+e^{-\alpha_{\tau} d_{\tau}}\right]}{\left[1+e^{-\alpha_{\tau}\left(d_{\tau}-R_{i j}\right)}\right]}
$$

Where $A_{\tau}$ is 1 for $\tau=s s \sigma, p p \sigma, p p \pi$ and 0 for $\tau=s p \sigma$. This leaves 12 other adjustable parameters $B_{\tau}, \alpha_{\tau}, d_{\tau}$ for $\tau=s s \sigma, s p \sigma, p p \sigma, p p \pi$. We fit this functional form to overlap data extracted from density functional theory simulations of a boron dimer using a hybrid functional and minimal basis set (B3LYP/STO-3G) using the GAUSSIAN03 ${ }^{28}$ program . Typical plotted output is shown in shown in figure 2 .

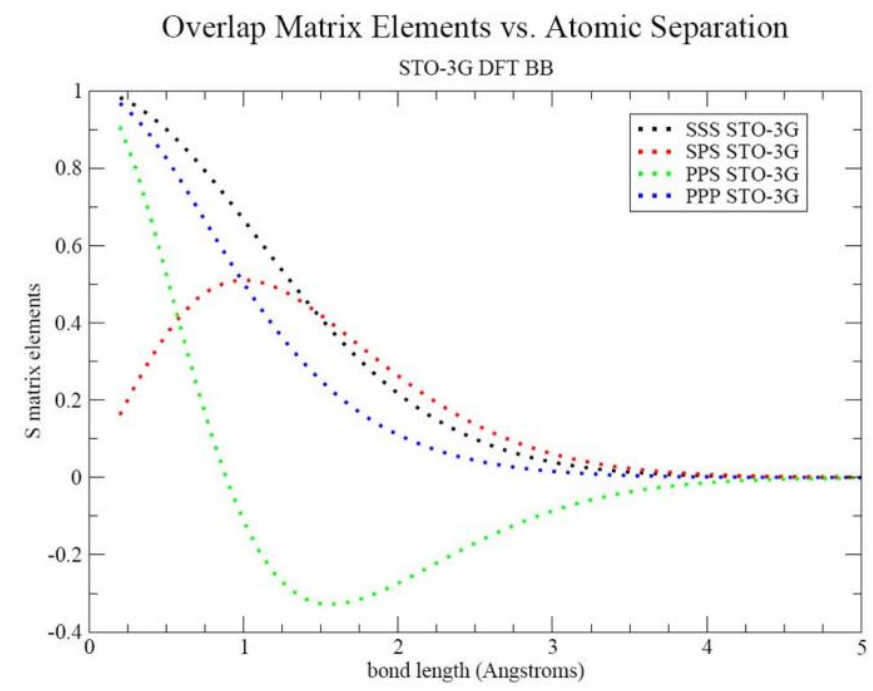

Figure 2. DFT overlap for boron dimer using a STO-3G basis set. 
We used the Levenberg-Marquardt algorithm ${ }^{29}$ to find the best fit parameters for our initial guess. It should be noted that depending upon the basis set used during a DFT simulation, widely varying dimer overlap curves are possible. For example the DFT results using the CEP-4G basis with a B3LYP functional have different nodal minimum and maximum locations as shown in figure 3.

Overlap Matrix Elements vs. Atomic Separation

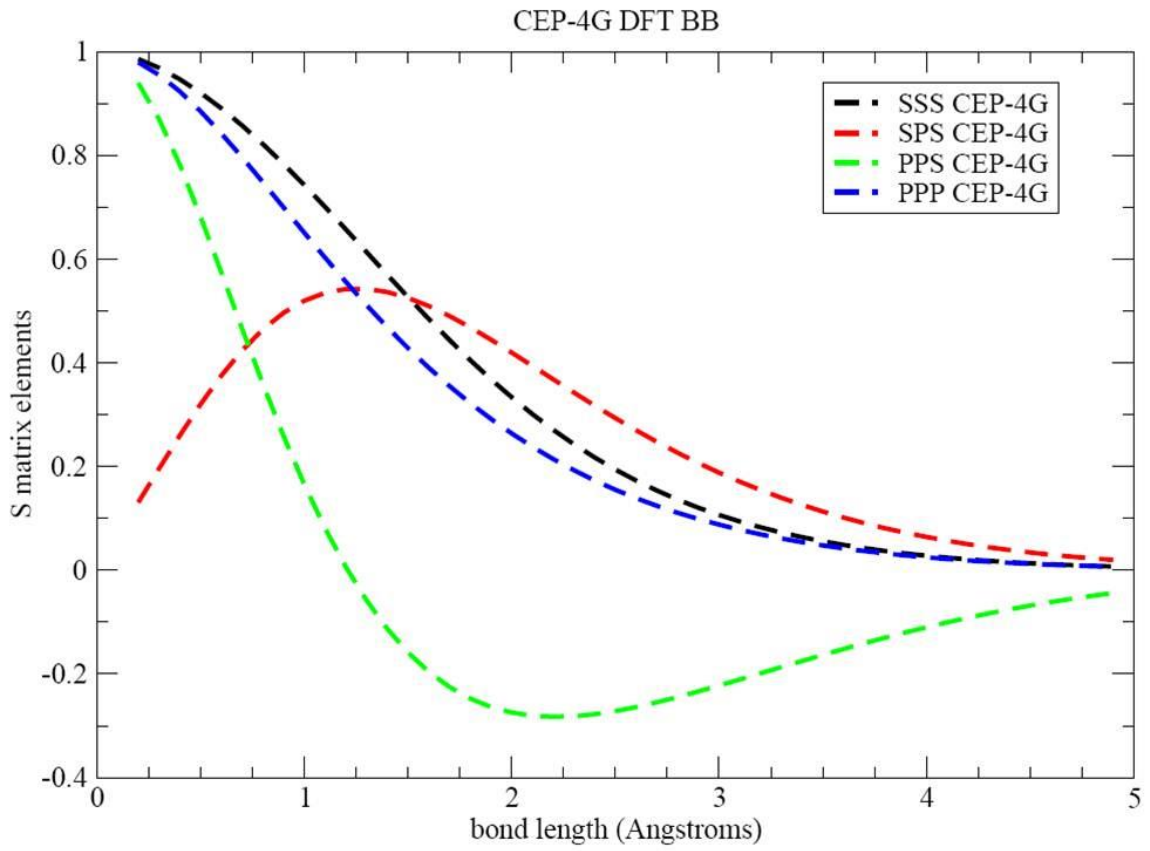

Figure 3. DFT overlap for boron dimer using a CEP-4G basis set.

We also compare the general shapes of the overlap functions of both DFT cases above with a tight binding result of Widany ${ }^{30}$ for boron in this plot. We note that all three results vary quite a bit, but the general shapes of the overlap functions are consistent as shown in figure 4. All three would be good initial guess for our overlaps because we will optimize the system after the guess 
parameters are inserted into our overlaps. The results indicated that the STO-3g dimer case was sufficient for an initial guess and was used initially for boron and phosphorus. It is highly recommended that this approach be used as an overlap guess for other elements that will be analyzed in the future.

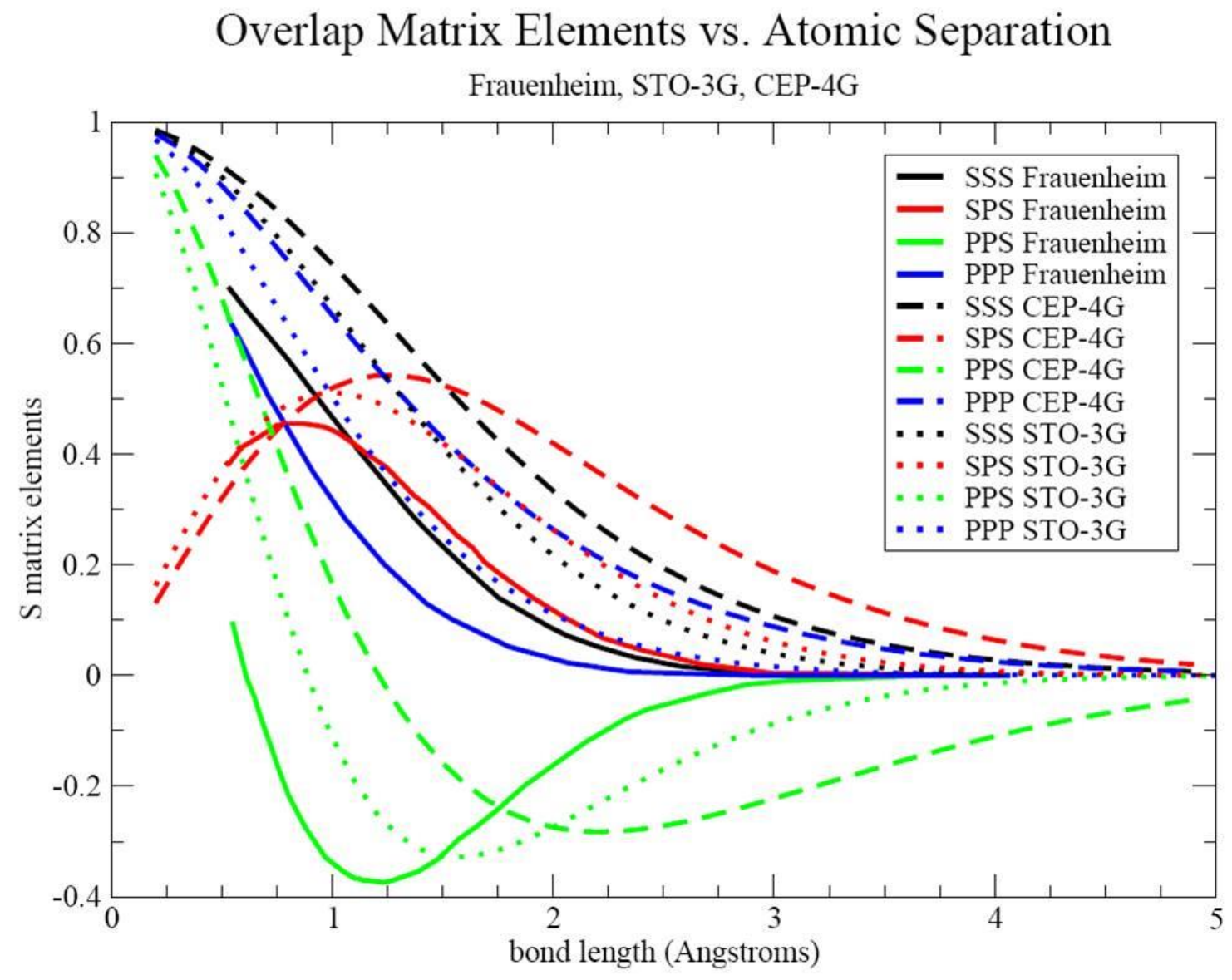

Figure 4.DFT dimer and tight binding overlaps

The overlap has three constraints that must be met when adjusting the parameters. The magnitude of any overlap curve cannot exceed unity. The overlap curve must decay to nearly zero in a finite number of bond lengths (the cutoff), for example by the $6^{\text {th }}$ or $7^{\text {th }}$ nearest neighbor there should be no overlap 
contribution. Note that the $\lim _{R \rightarrow 0} S_{i j, \tau}=1$ for $\tau=s s \sigma, p p \sigma, p p \pi$, and $\lim _{R \rightarrow 0} S_{i j, \tau}=0$ for $\tau=s p \sigma$. This was manually checked for every parameter set in question. The $B_{s s \sigma}$ parameter in the $s s \sigma$ overlap curve was particularly troublesome; during optimization this parameter would routinely force a condition where $S_{i j, s s \sigma}>1$ which is non-physical. To solve this problem, the parameter was constrained to $0.1<B_{s s \sigma}<0.3$. This resulted in huge computational time savings and should be noted for future reference.

\subsubsection{Initial Hamiltonian Parameter Estimates and Constraints}

The Hamiltonian contains 13 adjustable parameters (two fixed) in conjunction with the $12 \mathrm{SP}$ block parameters for the overlap functions. These parameters require an initial guess also. The values of $\varepsilon_{s}$ and $\varepsilon_{p}$ are pinned and not allowed to adjust at all, these are taken from the work of Mann ${ }^{17}$ to be $13.46 \mathrm{eV}$ and $-8.43 \mathrm{eV}$ respectively. The values of $\varepsilon_{s}^{\prime}$ and $\varepsilon_{p}^{\prime}$ are taken to be 1.5 times their non-primed counterparts and are constrained during optimization $\varepsilon<\varepsilon^{\prime}<2 \varepsilon$.

The initial guesses for the function $W_{i \alpha}\left(R_{i k}\right)$ are meant to mildly modify $\varepsilon$, for that reason $W_{s}^{0}$ and $W_{p}^{0}$ are initially set to zero and allowed to vary plus or minus small amounts during the optimization phase. $W_{s}^{1}$ and $W_{p}^{1}$ are exponential coefficients and dictate the decay rate of the strength of $W_{i \alpha}\left(R_{i k}\right)$, thus we expect 
them to fall off on the order of an inverse bond length; a reasonable guess is about $1 \AA^{-1}$ in both cases.

An initial guess for the $U$ Hubbard intra-atomic Coulomb repulsion is obtained from the work of Harrison ${ }^{18}(10.26 \mathrm{eV})$. This is allowed to vary $0.8 U_{i}<U<2 U_{i}$ in a soft manner, meaning that a weak penalty was enforced if the value neared the constraint. The Hückel exponential coefficient $\alpha_{k}$ is meant to be small so that the function itself does not grow rapidly; we then simply set it to 0.1 $\AA^{-1}$ and do not constrain it.

The initial guesses for the parameter $B_{Z}$ in $V_{Z}\left(R_{i k}\right)$ and $A_{N}, B_{N}, \alpha_{N}, d_{N}$ in $\Delta V_{N}\left(R_{i k}\right)$ are a bit trickier. Since we are looking for a guess and great accuracy is not needed, we use the parameters found for silicon in a previous formulation ${ }^{16}$. This meets our initial guess requirement, the functional shape must be similar in the initial guess, but that is all that is required. We do not want to assume too much about our Hamiltonian or overlap in the beginning stages of fitting. Thus, the parameters for $V_{Z}\left(R_{i k}\right)$ and $\Delta V_{N}\left(R_{i k}\right)$ are not constrained in any way. But the general shape will be inspected as the code runs to make sure that these functions decay as they get large. These initial guesses should get the user into a reasonable part of the parameter space to start a more complete optimization. It can be very computationally time consuming to optimize a set of parameters in this 25 parameter space (especially with such a heavily nonlinear problem such as this). Small changes in parameters can impart very large changes in the bonding model as noted previously. Because of this, all significant digits must be 
presented to the molecular dynamics code when testing and running various systems. Truncation of parameters can lead to incorrect results. All optimized parameters are reported to the $8^{\text {th }}$ decimal place. This must stay consistent when sharing parameter information between the fitting code and the molecular dynamics code, and between users of both codes when sharing and comparing parameter sets.

\subsection{THE GENERAL APPROACH TO PARAMETER OPTIMIZATION}

Once reasonable parameter starting guesses have been obtained, we can test the Hamiltonian with a molecular dynamics package of our choice and see how well our model performs. If it performs sufficiently we are done and can use this parameter set to run our simulations. This will most likely never be the case though, because our crude guesses will inevitably give poor results. We seek to improve our parameter set via a two stage methodology. First we will fit our parameters using a Levenberg-Marquardt routine from IMSL ${ }^{31}$ (D_UNLSF) imbedded in a very complex fitting code written by Dr. Christopher Leahy in the CMT Group at the University of Louisville ${ }^{15}$. We will then perform a secondary robustness check (defined later) manually using a secondary molecular mechanics program also written by the Condensed Matter Theory Group at the University of Louisville. In both cases we have a database standard comprised of GAUSSIANO3 ${ }^{28}$ and $\mathrm{VASP}^{32}$ ab initio electronic structure results. We will compare our SCED-LCAO results to the results obtained with these programs. 
When we chose to compare two electronic structure methods, and fit one to the other, we are faced immediately with a series of dilemmas. Notably we must decide upon trades offs that must be made for computational expediency. We cannot assume that it will be expedient to fit 25 parameters, accurate to 8 decimal places, in a strongly nonlinear system. We suspect that small changes to the parameters will impart huge changes to the energy and general bonding nature of any system we attempt to model as stated before. Given that, we anticipate that every possible effort should be incorporated to reduce computational time and memory allocation. I will outline a few of the major time savers incorporated while seeking a solid set of boron parameters that will prove to be maximally transferable and reliable under the afore mentioned approximations in chapter 1 .

\subsubsection{Convenient fit properties}

We are faced with the task of utilizing a Hamiltonian and overlap matrices with semi-empirical parameters inserted in the matrix elements. For a given set of parameters, one can describe a molecule with a set of spatial coordinates as input, and then calculate the total energy of molecule or the binding energy. This is one of the most fundamental calculations in electronic structure theory. We could also calculate the band structure of a periodic system given a set of coordinates, but at larger computational cost. We also run into the problem of where are we going to get band gap results to fit our method? For example, DFT is notoriously unreliable in predictions of the band gap for silicon. 
The preferable fitting properties for our scheme will be the properties that are easiest to find, and easiest to calculate. This turns out to simply be the binding energy and structure coordinates of a given system. These properties are easiest to obtain from current ab-initio software, but are quite difficult to find experimentally for small clusters. In fact in many cases, they are not available at all. The band gap is usually easy to find experimentally but getting the exact thermodynamic properties such as pressure, temperature and crystal structure may be challenging. Due to these challenges, we will use the most accurate abinitio methods that can possibly be found within reason. There are limits to this approach, which will be discussed later in this chapter. We will extract energy and spatial structure from the ab-initio simulations (not experiments), and a given set of parameters and coordinates. Energies will be compared between our SCED-LCAO results and the ab-initio results. Other properties such as heats of formation, band structure, melting point, band gap, resistance, etc... will not be

included as properties in our database fit due to the large computational effort to calculate such properties. To do so, we would have to alter geometries and move through our 25 dimensional parameter space during the fitting process and that would be too computationally costly.

\subsubsection{Available Commercial Software}

The commercial packages GAUSSIAN03 and VASP were used exclusively for different reasons to create a database. For extended structures with periodic boundary conditions the VASP plane wave based DFT code proved 
to be the most cost effective tool for database generation. For small finite clusters of atoms, the Gaussian basis set based code GAUSSIAN03 was used.

Both DFT and Coupled Cluster methods are included in the GAUSSIAN03 code making it preferable for cluster generation purposes in the database. GAUSSIAN03 can treat periodic boundary conditions, but the usage of a Gaussian basis set proved to be computationally cumbersome for extended systems, and quite slow. VASP was clearly superior for extended systems with its plane wave basis set, making it fast and easy to construct bulk system databases. Using both packages, a cluster and bulk database of boron structures was created. The only caveat is that we now have a hybrid database of plane wave basis sets with one type of functional, mixed with a database comprised of a Gaussian function basis set with another type of model chemistry. The assumption is that both are accurate beyond our semi-empirical method and any inconsistency between the two is minimal in impact upon our fitting process. This may or may not be fully accurate and remains an open topic for future exploration. In the meantime we act upon this premise out of necessity and justify this assumption based upon similar results obtained for a few test cases in previous work $^{16}$ in the Condensed Matter Theory Group at the University of Louisville.

\subsubsection{Current Literature: A starting point}

Ultimately we want to do three things initially during our parameter quest. We want to have good initial guesses for our fitting parameters. We want to rapidly and accurately build a cluster and extended phase database. And, we 
want to build an enlarged database of far larger clusters to check our parameters at a later time. There are numerous articles ${ }^{33-38}$ based upon small cluster studies, and should be used judiciously to expedite the database creation. Most studies compare energies of various small clusters using different methods and basis sets to arrive at potential low energy candidates for the ground state of a given assembly of atoms. For example if we have 5 atoms of boron, using a particular method and basis set, we will arrive at a given energy and set of bond lengths and angles. It is to your advantage to use this literature as a starting point, but not a substitution for database creation. Any information like this found in any journal must be checked and rechecked for accuracy. There is only one way to do this, and that is by actually running the potential cluster or extended phase in your software and extracting spatial and energy information using your computer. Many of these clusters studies in the current literature represent extensive work and computational effort, but, it is noted that errors in literature do occur and simply copying the information out of the literature and placing it in a database (without testing) is unwise. The database used for boron for example contains no experimental data; all input is generated from computational models using either density functional theory or coupled cluster theory, thus it must be accurate.

All suspected candidates for the database were re-run using commercial software, even if the literature lists energies, bond lengths, angles, etc., which in most cases it does not. All that is really needed is an image or well defined space group of a possible candidate cluster or unit cell to run the simulation. The 
person constructing the database can then create user input files that look similar to the candidate clusters or candidate unit cells. The boron database contains mostly, but not all, low energy clusters. It can be extremely difficult to simply guess at what a low energy configuration might look like for example 8 boron atoms. It is very likely that someone has already explored this, and has found (if not the lowest energy structures) some very low energy structures that would make excellent candidates for your database.

Although we could build a database of all guess structures, high energy structures and fictitious structures, it is noted that in the case of boron when this was tried ${ }^{39}$, results were far less accurate in terms of total consistency with DFT than when a great sample of mildly high and lowest energy structures were included in the database. It is also noted that the inclusion of very high energy structures (more than about $0.1 \mathrm{eV}$ per atom above the accepted ground state structure) may actually pollute the database with low accuracy information, making it more difficult to fit the parameters to the database. This assertion may be justified from the nature of density functional theory in general. It is a variational approach and thus should most accurately model (for a given number of atoms) the lowest energy isomer for a fixed number of atoms. Given that, we expect that the most accurate information for our database comes from the lowest energy isomers. Accordingly, the inclusion of very high energy clusters that are simply theorized proved to be problematic when they were included in the boron database. After numerous attempts ${ }^{40}$ it was then determined that they were not the best candidates and removed from the database. Although it is also 
important to include higher energy structures in the database, once the energies become significantly higher, fit quality was observed to reduce dramatically.

\subsubsection{A Standard vs. a Fit}

After fitting to a standard (in our case DFT and coupled cluster simulations), we note the fits are not perfect fits in all cases compared to the standard. The fit may be close on some, and not so close on others. Due to this fact we have to proceed carefully if we want to improve the quality of the fit. We have several choices in how to proceed. We can continue to run the fitting code in hopes of getting a better fit to all of the properties or we can steer the fit to sacrifice some fit quality for some properties in favor of a better fit for all properties. We can choose to define a quality to our fit, and stick rigidly to that or not. But, no matter what we fit to in our database, or how we define quality, we will fit to some properties better than others. It is not guaranteed that with increased computational time, it will be possible to arbitrarily fit our set of parameters to the ab-initio database assuming we compare the exact same energies given the same bond lengths and angles. This is because we are not guaranteed convergence to a perfect fit and still retain the appropriate shape of our phenomenological functions.

In practice we have defined a quality factor which we want to continuously reduce, it is a residual that is defined as a factor that decreases as our fit quality increases. The parameter optimization is guided by minimizing this quality factor as the code runs. At some point one will notice that the residual is not reducing anymore, even though the code has been running for a very long time, maybe 
months. At this point the phenomenological functions must be inspected for shape consistency (e.g. an overlap cannot exceed unity), and a secondary robustness database (a computationally demanding database) with much larger molecules will be utilized for a secondary fitting to inspect performance.

We will also not be guaranteed uniqueness of our parameter set. It is very possible that one can come up with a set of parameters that leads to a fit of the database very well, and another person can come up with a completely different set of parameters that also fit the database very well. This is why the secondary robustness database is necessary. We will have many sets of parameters, all will fit the database fairly well, all will have a high quality fit, all will have a low residual, all will look appropriate, but some will perform far better than others when we test the parameter set on far larger molecules. It is in this robustness phase of the fitting that we realize that simply having a good low residual fit in our database will be insufficient in many cases.

\subsubsection{Reliability of the Proposed Metric}

Once the residue has been reduced in the fitting code through optimization, we are optimally fitting the best we can with the database that we are using. As explained above, there will not be a unique set of parameters after this fitting process. As stated earlier the Hamiltonian and overlap are sensitive to small changes in the parameters. This may not be apparent from simply looking at parameters generated from the initial database fitting. This necessitates going further into the fitting process outside of the database. 
Once we try the Hamiltonian (based upon any post-fitted parameter set we determine) on any system not included in the fitting set, problems may be seen when comparing our results to ab-initio results for systems not included in the fitting set. In other words, our Hamiltonian and overlap may not be transferable nor are they reliable, even though we have a good fit for the database. This would be a problem but we note that the parameters sets are not unique from the database fit. This means a different parameter set may possibly be far more reliable and transferable. Because of this, we go to a secondary parameter check called a robustness check, basically it is a secondary fit, but is far more time consuming and computationally demanding. If the parameter set does not meet the requirement of a low residual during the fitting optimization (and other quality checks) we will not consider it for the robustness check. We arrive at multiple parameters sets by running the fitting code simultaneously on many (hundreds) of machines. We will thus have hundreds of parameters sets all of varying quality, all need to be inspected rapidly, and all must fall into one of 2 categories: worthy of the robustness check or not worthy. This can be challenging to the point that the problem becomes a large code management and data extraction dilemma. We thus view the initial fitting code as a provider of three functions:

1) It will provide us with a low residual set of parameters based upon a fitting database, and a single processor.

2) It will provide this set of parameters in a fairly timely manner.

3) It will provide many of these sets, if we have many processors. 
But it will not necessarily provide the final best set in a timely manner, only good candidates, even if we have a lot of processors.

The cluster fitting database for which all parameters will be fitted will contain small clusters. Our cluster robustness test may contain intermediate to very large clusters (i.e. clusters that greatly strain the possibility of use by abinitio methods). The design of these databases is not arbitrary, and in practice it was demonstrated to require a great deal of time.

\subsubsection{Gaussian Type Orbital Basis Sets}

For clusters it is recommended that a database be created that uses orbital basis sets, in this case Gaussian basis sets as shown in figure 5 .

For extended phases it is recommended that one use a plane wave approach. Orbital basis sets are localized and make more sense for small structures; plane waves are delocalized and are more appropriate for extended crystals and surfaces.

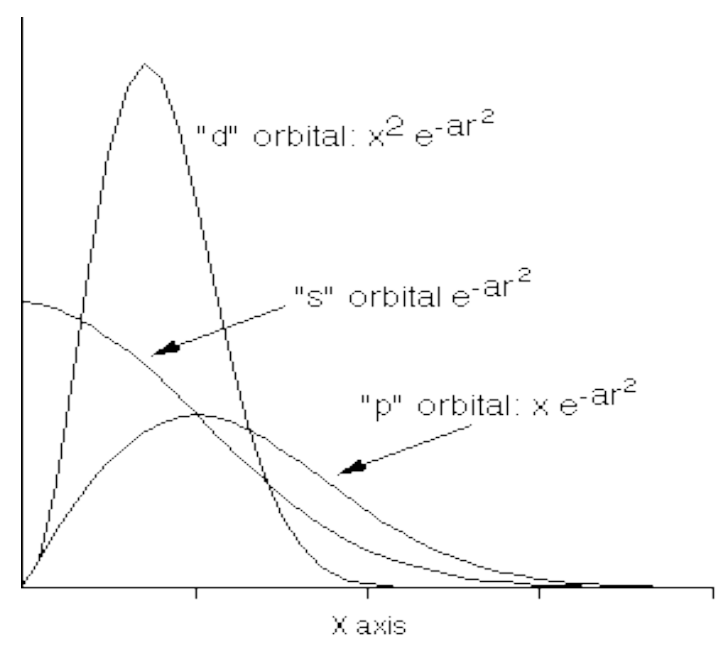

Figure 5. Gaussian type orbitals 


\subsubsection{Model Chemistries}

For extended structures the DFT method is recommended for database construction. Due to the computational facilities available at the University of Louisville at the time of this study, we were not able to use all coupled cluster, configuration interaction methods or some other very accurate post Hartree-Fock methods for all cluster calculations but were able to use them for smaller cluster calculations. At that time the largest single point coupled cluster calculation I could do was a 12 atom boron calculation. If this was exceeded, all the memory on the shared SGI machine (i.e. Canary) was used and crashed. However, 12 atoms is sufficient for the boron database. For extended phases the same machine was used to run DFT/GGA with no problems because the unit cell was sufficiently small and DFT is far less computationally demanding than most post Hartree-Fock methods.

\subsubsection{Specific Basis Sets and Cost}

The basis sets developed by Dunning and coworkers ${ }^{41}$ were utilized to generate the cluster database. The basis sets were designed to converge systematically to the complete-basis-set (CBS) limit using empirical extrapolation techniques. For boron, the basis sets are cc-pVNZ where $\mathrm{N}=\mathrm{D}, \mathrm{T}, \mathrm{Q}, 5,6 \ldots$ ( $D=$ double, T=triples, etc.). The 'cc-p', stands for 'correlation-consistent polarized' and the ' $\mathrm{V}$ ' means they are valence-only basis sets. Included are increasingly larger shells of polarization (correlating) functions (e.g. d, f, g) these sets are the current state of the art for correlated or post-Hartree-Fock calculations. In particular the aug-cc-pVTZ triple zeta augmented version of the basis set with 
added diffuse functions was used exclusively. This slows down the calculation, but, smaller basis sets are less accurate and for the boron database only highly accurate basis sets for small clusters. This ensures that inaccuracy cannot easily originate within the small cluster database.

\subsubsection{Fixing Symmetry for clusters}

During SCED-LCAO optimization, the lower the number of degrees of freedom, the faster the fitting occurs. Symmetry is fixed to the lowest number possible for an isomer.

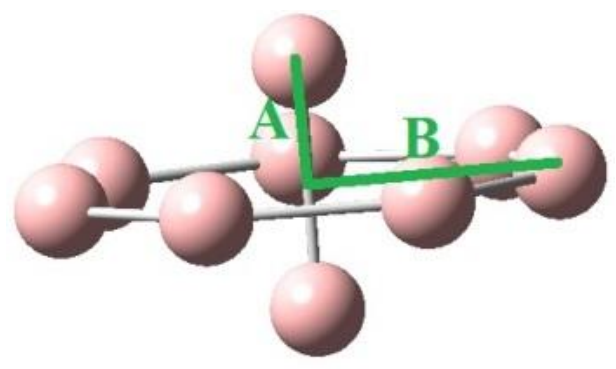

Figure 6. fixed symmetry during the geometry optimization

For example in figure 6 , the cluster has 2 spatial degrees of freedom, or two spatial properties A and B. A third property is the energy, so this cluster has three properties in total. During the fitting stage the geometry is optimized by fixing this symmetry. This greatly increases the speed of the optimization. If a molecular dynamics routine (MD) was used instead, and the cluster could wiggle around in all directions, parameter fittings would require a much greater computational time. 


\subsubsection{Initial extended database}

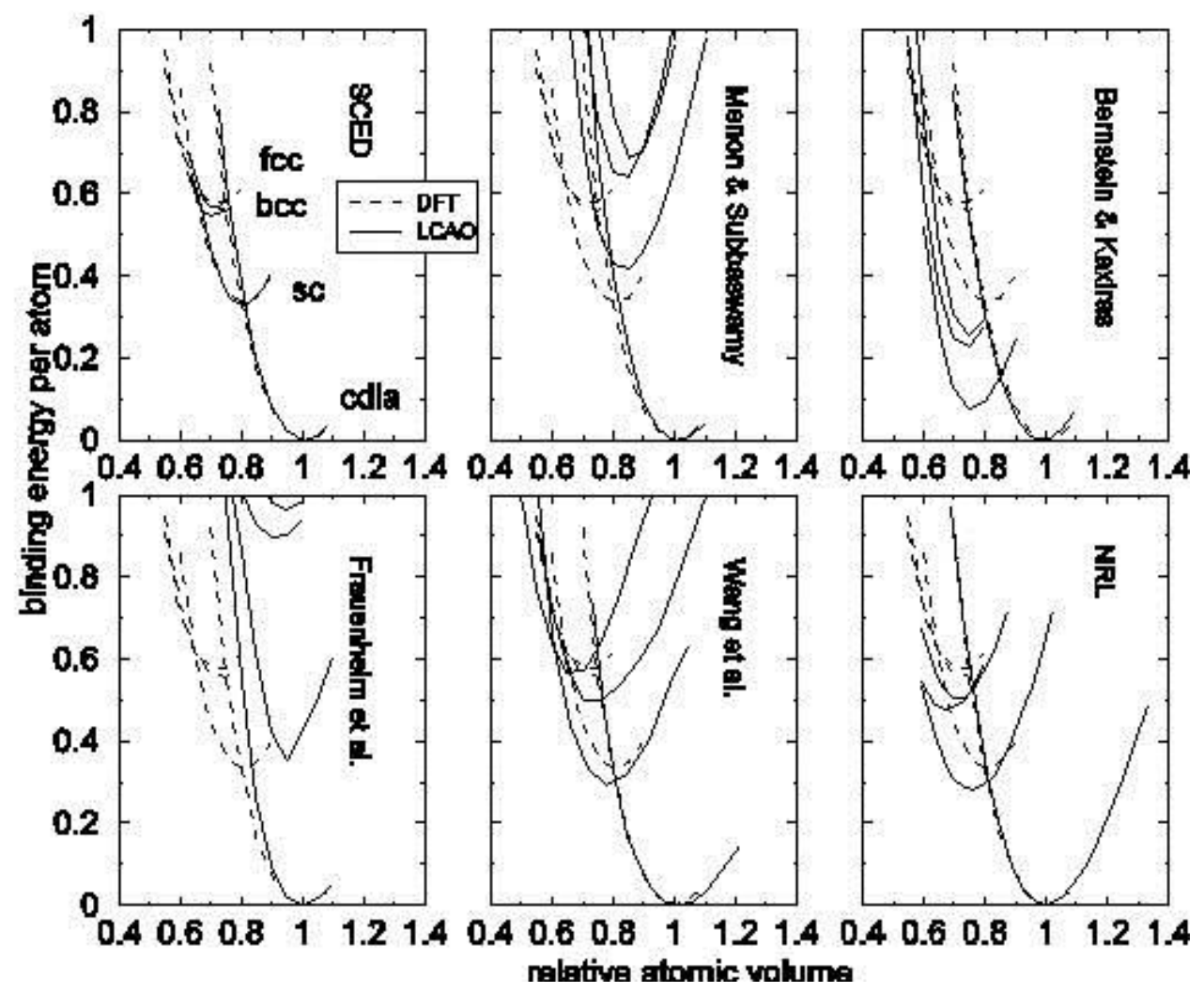

Figure 7. simple phases of silicon

Due to the success of the silicon Hamiltonian ${ }^{1}$ based upon comparison of the simple phases of silicon with other semi-empirical electronic structure research groups ${ }^{42-45}$, it was initially decided to try the exact same phases for boron. The initial successful plots for silicon are shown above in figure 7. By comparison, we have an initial plot for boron, shown below in figure 8 . This fit is exceedingly accurate, which gave us the confidence that we could successfully model boron. 
Bulk Cohesive Energy vs. Relative Atomic Volume

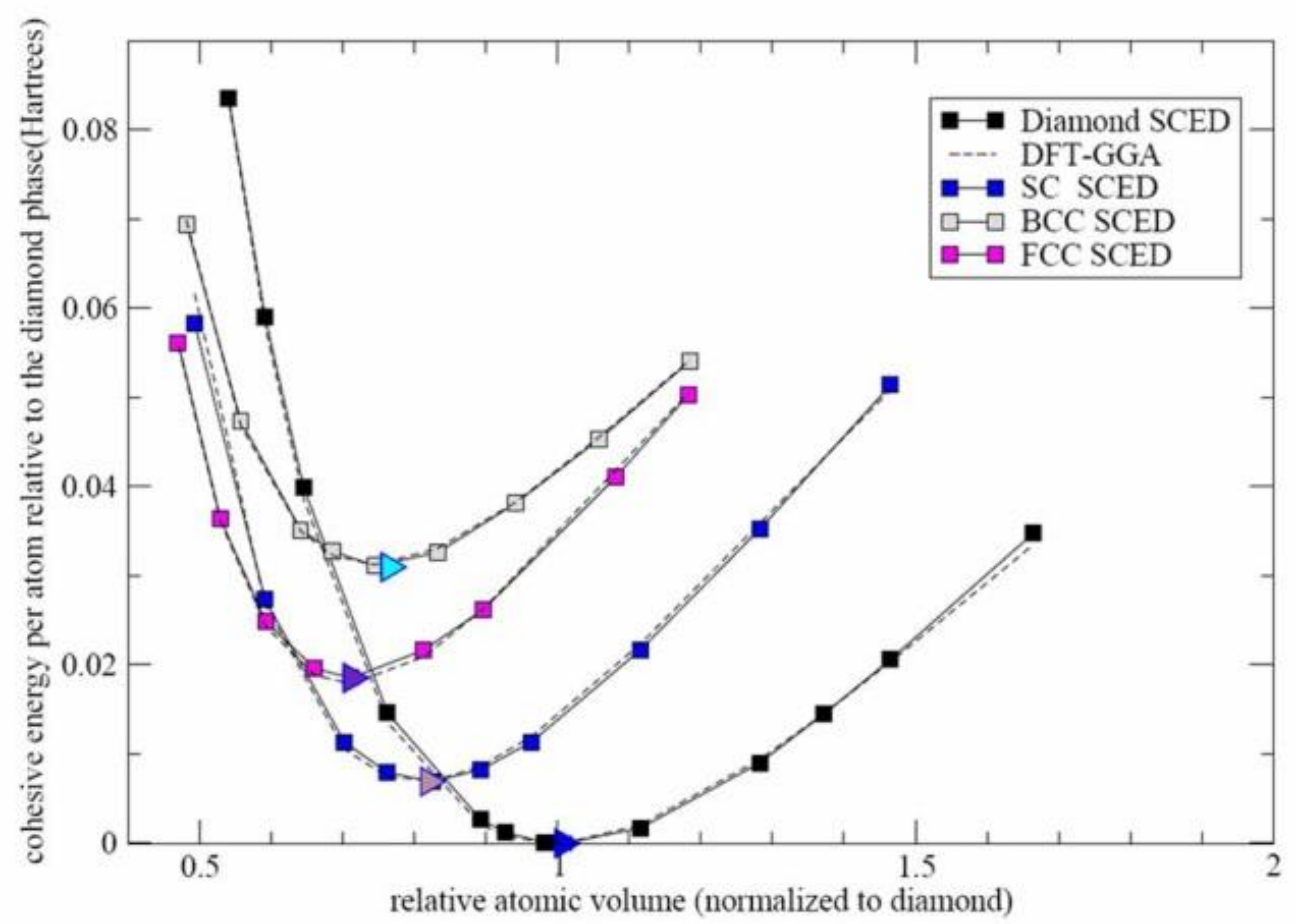

Figure 8. binding energy vs. relative atomic volume for boron

\subsubsection{Convergence}

The time progression of the quality of our fitting parameters is loosely outlined below, in figure 9 .

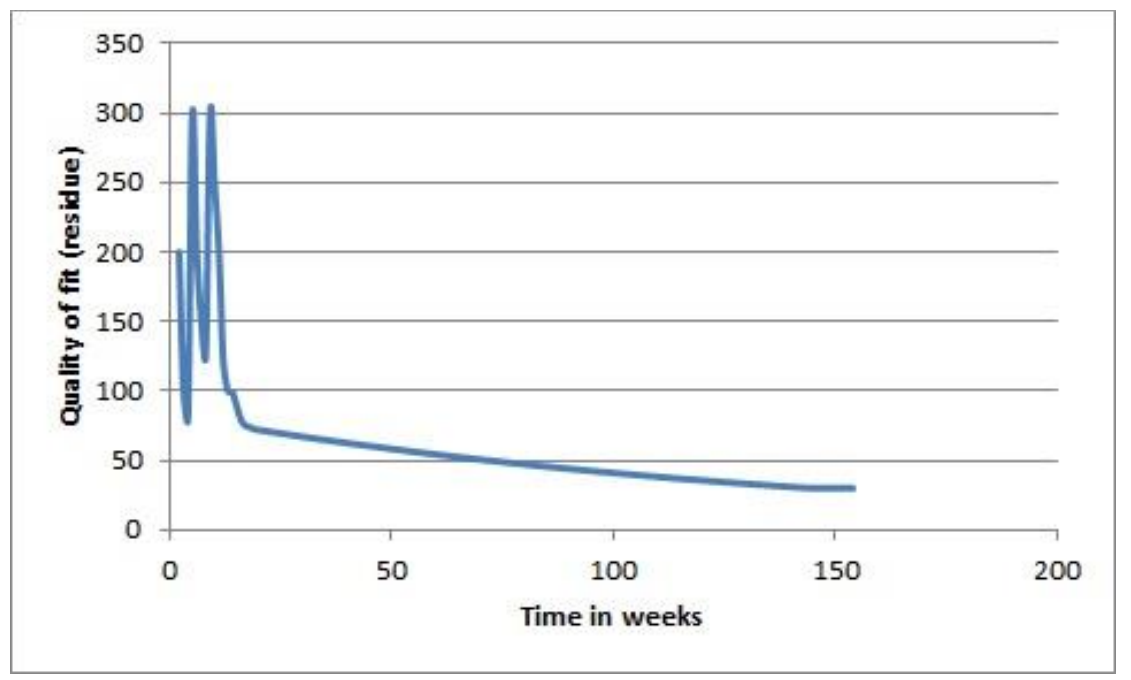

Figure 9. Fit progress 
A lot of progress is made quickly, but as the database is increased, and more and more tests are performed it gets increasingly difficult to lower the residual. The total time effort for boron was about a 5 year investment in parameter acquisition, while phosphorus was about a 6 months. If parameter acquisition was attempted for different element today, the investment would be around 4 months. The 5 year time frame for boron has more to do with refining a testing methodology than it does with actual computational time.

\subsubsection{Self-consistency routines}

The fitting code and MD code are fully self-consistent. Charge redistribution is fully taken into account. The general outline of this is shown below in figure 10 .

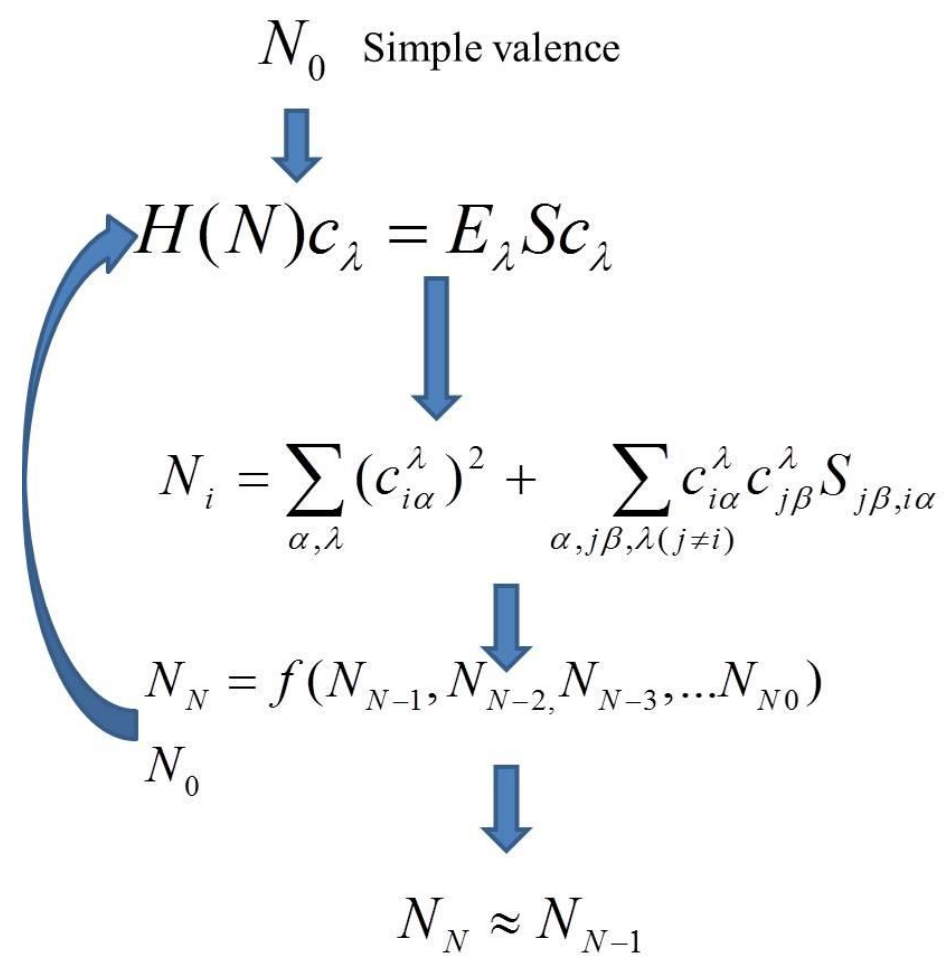

Figure 10. Self-consistency methodology 


\subsection{PARAMETER FITTING}

\subsubsection{Stage 1: A small cluster database}

Initially when executing the fitting code one will encounter two serious problems. The first problem will be the inability to achieve self-consistency in the calculation. When this occurs the calculated residual is meaningless, so the code is halted and an attempt is made by some mechanism to alter the initial parameters until self-consistency is achieved. The second problem that will be encountered with the phenomenological version of the overlap calculation is the calculated overlap matrix may not be positive definite. This likewise will also halt the code. Note that this problem is solved in an alternative model that relies upon an orbital approach to calculate the overlap, which was not used for this project. But in either code, and in most electronic structure codes, achieving selfconsistency in a rapid manner is a difficult problem.

To abet this dilemma the approach taken was to find out if either of these two problems is present initially in our parameter search methodology. If they are, then we need to rapidly change the parameters. There are five issues that will slow the fitting process, sometimes to a point that the code will not run at all. Issue 1 is the size of the largest cluster used in the fitting database. For example, is the largest cluster 6 atoms or 20 atoms? Issue 2 is the total number of clusters. If for example, the total number of clusters increases from 6 to 40 the fitting process will be far slower. Issue 3 is the total number of properties in the system. Properties for example, would be spatial properties such as bond length or 
functions of bond length, fixed angles or energy of the cluster. Minimally we want the total number of properties to equal the total number of fit parameters in our system, although this is statistically insufficient. However, realistically, by the time we have fully filled the boron database, we will have approximately 5 times more properties than fit parameters in our Hamiltonian and overlap combined. Issue 4 is the inclusion of bulk extended phases into the fitting process. The geometry optimization routine that was used in this body of work is significantly slower for the extended systems than it is for small clusters, this can greatly slow down the processing time especially if the unit cell of the extended phase is large (greater than approximately 15 atoms). Issue 5 pertains to how many processors we have at our disposal. If we run the same parameter fitting routine with differing initial random seeds on different processors we can move through the parameter space $\mathrm{N}$ times faster ( $\mathrm{N}$ being the number of processors). In this sense a large distributed machine is ideal. The problem with doing this is that we can wander through the same part of the parameter space over and over again, wasting valuable computation time. But, in practice this problem is less of an issue because the probability of moving into exactly the same part of parameter space is low due to the large size.

We should start with the best guess possible for our parameters. We should also place boundaries on these parameters to keep them from moving into a non-physical realm. But, the self-consistency and positive definite issues need to be addressed first. The fastest approach is to initially minimize the fitting database to the bare minimum that is required for the code to run, we will expand 
this later. In terms of the 5 issues listed above, we will choose our initial fitting scenario as follows. (1) The largest size cluster fitted initially was 7 atoms in size.

(2) The total number of clusters was 9. (3) The total number of properties was 35.

(4) There was no extended bulk phases included in this initial phase. (5) The number of processors used was the maximum number available at the time, approximately 30 . The database initially included only the following 7 clusters as shown in figure 11.
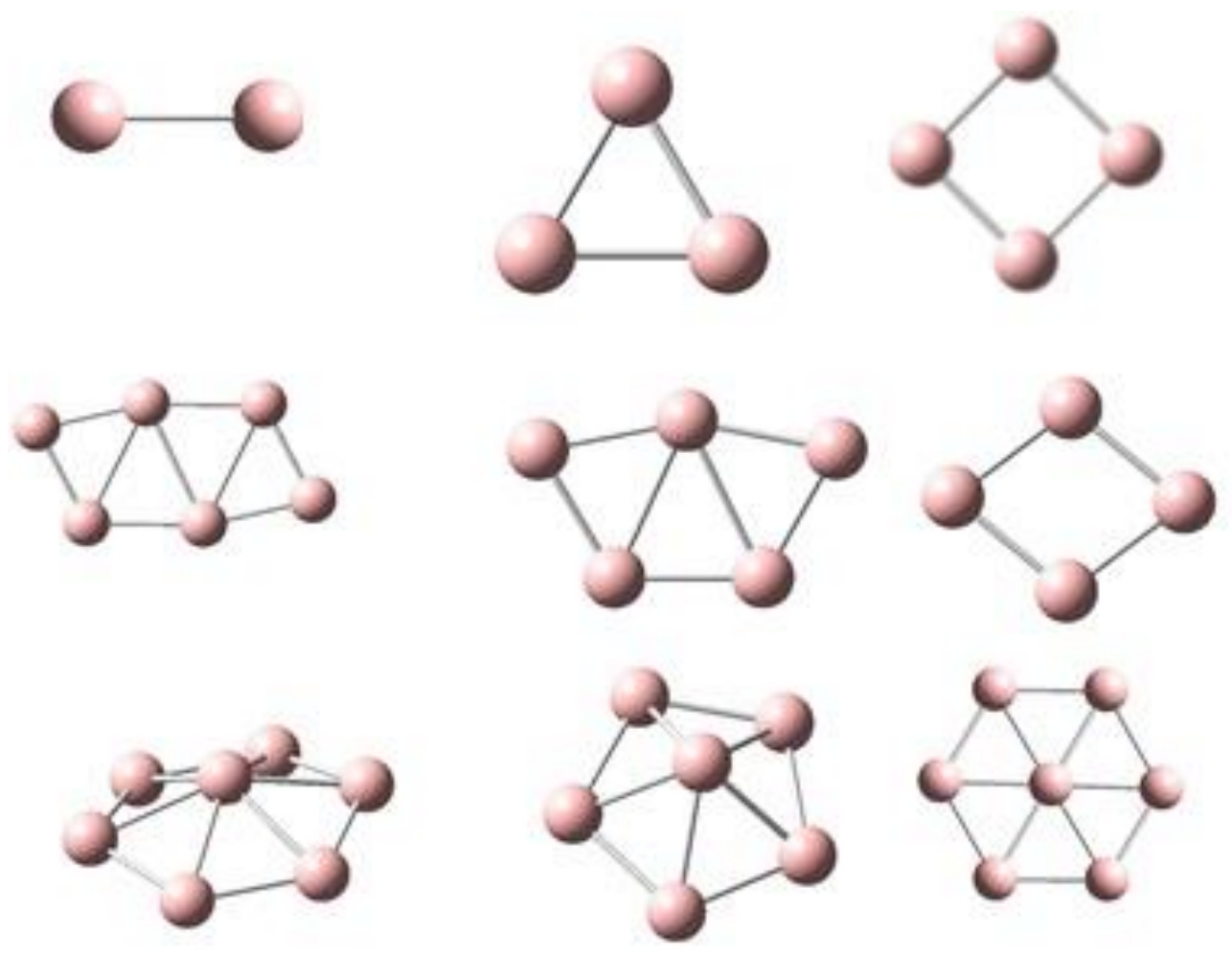

Figure 11. Clusters initially used for fitting.

Even though our initial guess for the boron parameters were carefully thought out, many of the processors failed to produce a fairly low residual, and many instances the code halted completely. But, out of the 30 plus processors that were running, at least 6 produced a residual ${ }^{15}$ lower than 130 (a residual of 
scale in the mid-30s is the goal). Of these 6 sets of fitting parameters several characteristics of the overlap were inspected. One issue was the shape of the overlap, if it was not shaped appropriately that parameter set was discarded. The final boron parameter overlap curves are show below in figure 12. All $P$ block element overlap curves should have these general shapes.

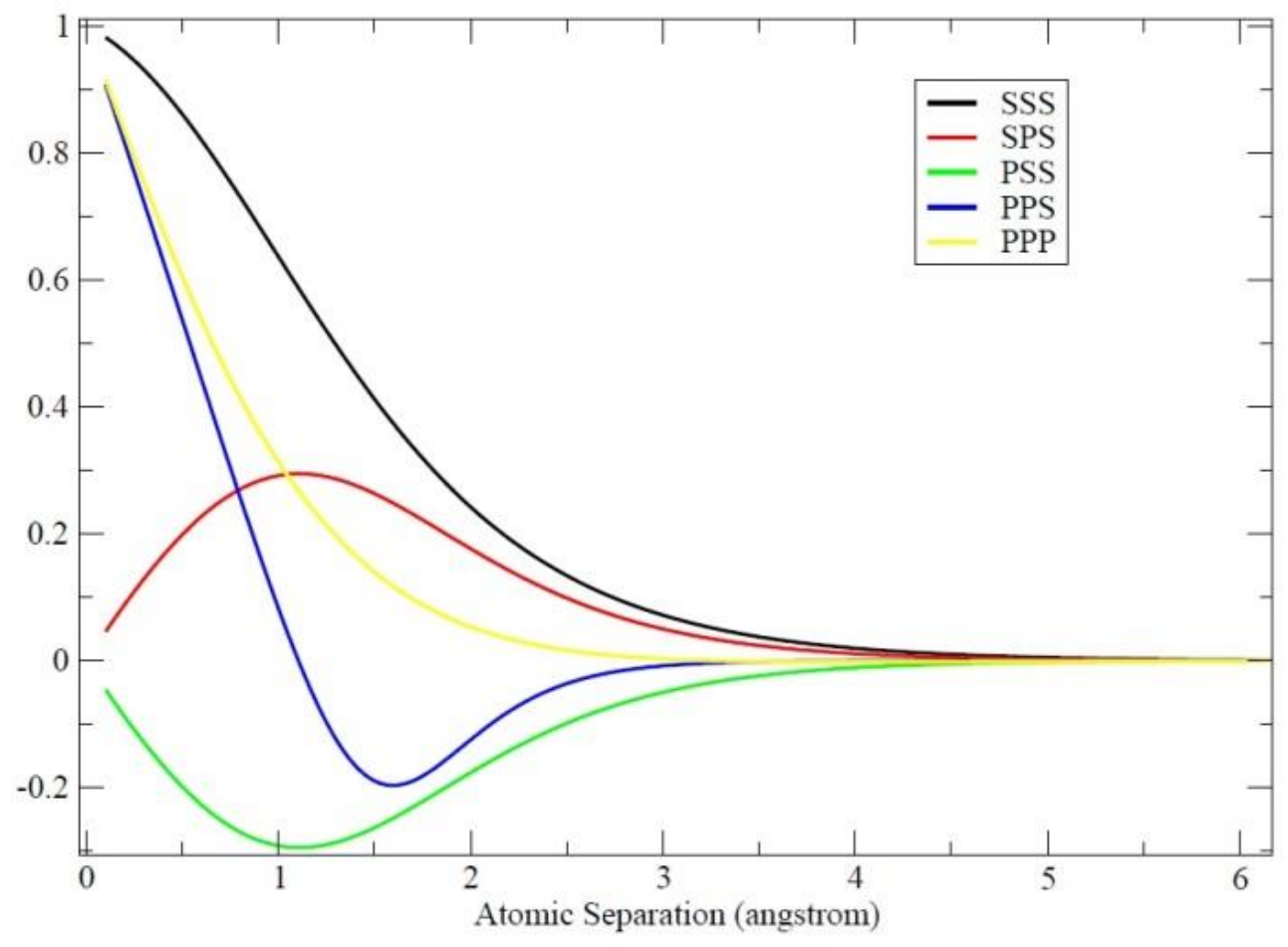

Figure 12. Overlap functions of boron (optimized shown)

The Hamiltonian curves should near zero around the cutoff point defined within the program. This must be manually checked. This criterion supplies a quick spot check of the parameters themselves. In figure 13 below the final optimized boron Hamiltonian curves are compared with the boron Hamiltonian curves from the work of Widany ${ }^{30}$. Note the curves are similar in shape. 

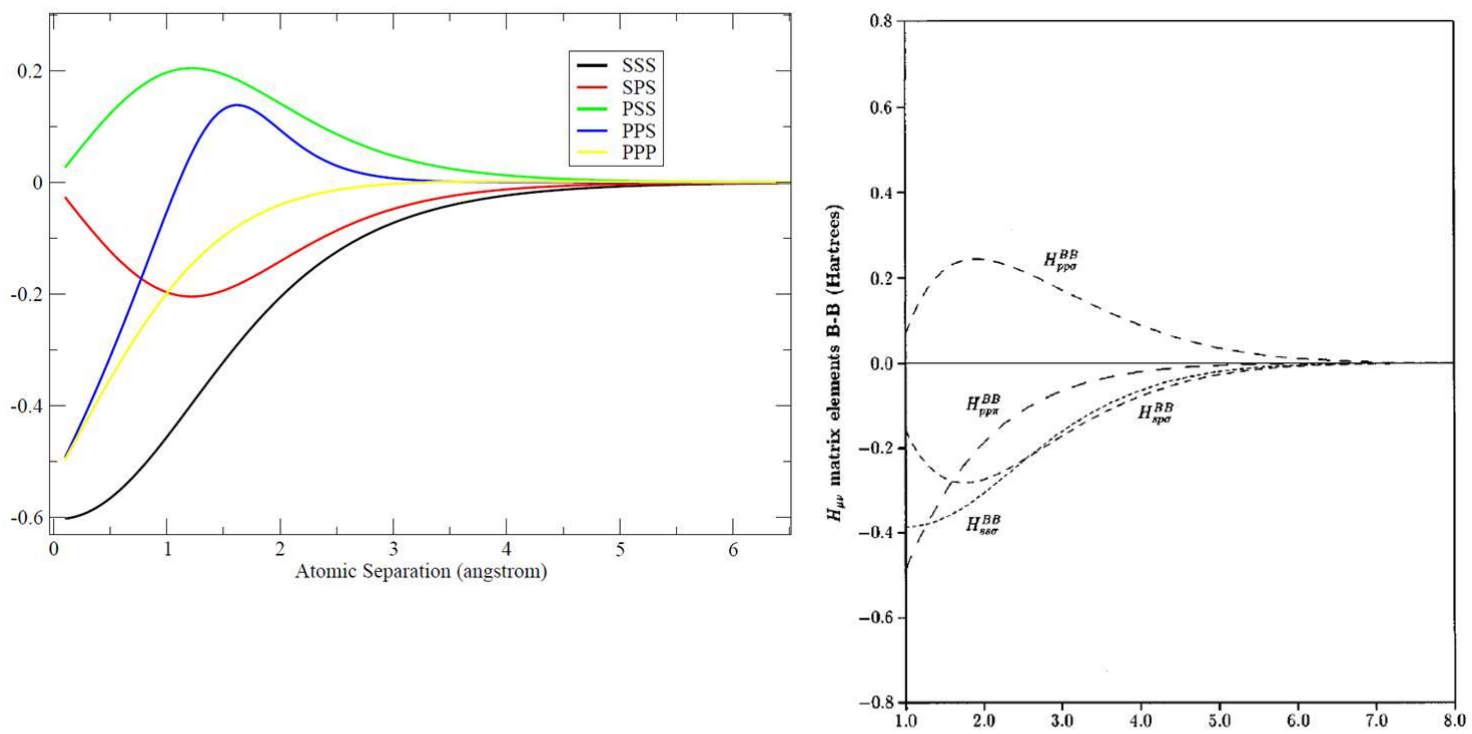

(a)

bond length (units of $a_{B}$ )

Figure 13. General shape of the Hamiltonian

If the parameters floated far out of their boundaries with high penalty, that parameter set was discarded. By the time all checks were made approximately 3 parameters sets were retained as candidates for the next stage in the fitting.

\subsubsection{Stage 2: Addition of a fictitious sets of extended phases}

From stage 1 approximately 3 parameter sets were retained. Copies of each parameter set are made and given different initial seeds. We will also expand our database to include phases of boron not found in nature; these are low probability or fictitious phases ${ }^{46}$, such as cubic boron. Generally only the two most common phases of boron are prepared in laboratories, alpha and beta boron. Simple phases of boron are not common and would be considered very high energy states. But for our initial version of our extended database of boron simple phases were included because of their small unit cell, see figure 14 . 
These extended phases of boron were calculated using DFT with plane wave basis set. The simple phases included: simple cubic, face centered cubic, body centered cubic and the diamond phases of boron.

Bulk Cohesive Energy vs. Relative Atomic Volume

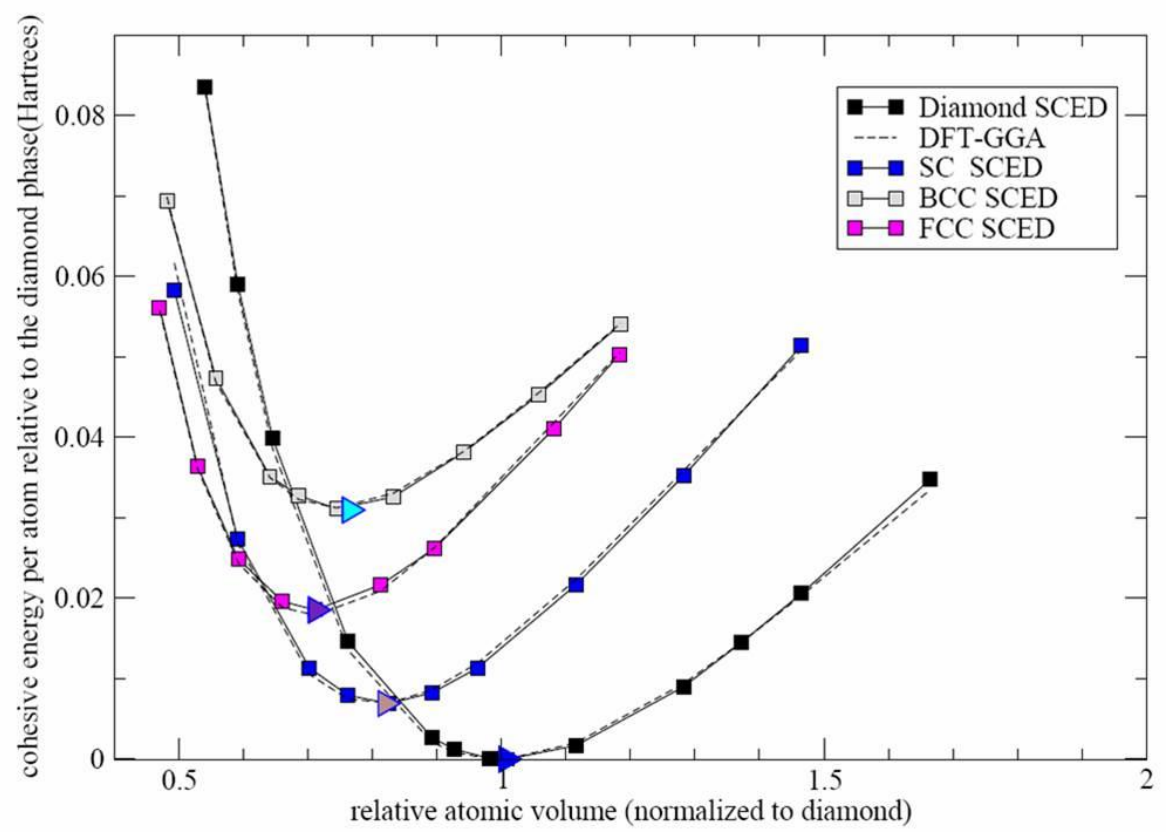

Figure 14. Fictitious phases of boron

The volume is increased homogenously by a multiplicative factor (i.e. a breathing mode). Once these phases of boron are included in the database, and all copies of the parameter sets are made with different seeds as stated above we proceed to the next stage of parameter fitting. All simulations will now run for as for long as it takes until a significant number of sets of parameters are found that can reach the following criterion:

- Achieve self-consistency;

- Overcome the problem of the non-positive definite overlap; 
- Have well shaped overlap functions;

- Have an appropriate tail cut-off on the Hamiltonian;

- Have reasonable values for the fit parameters; and

- Achieve a low residual for the extended phases and the clusters.

As parameters sets were found that achieved every one of these constraints, 26 copies of this parameter set were made, all given different initial seeds, and rerun on different processors. The lowest value residuals that still meet all the above constraints were retained. I call this process "parameter distillation" and this technique of expanding the database, copying the parameters, creating new random seeds, inspecting and retaining the best results is continued throughout this project until no improvement can be found within a reasonable amount of time (i.e. 1 to 2 months). At this point we are ready to move to the next stage of expanding our database with larger clusters.

\subsubsection{Stage 3: Addition of larger clusters}

Once a candidate set of parameters has been obtained with a residual below 50 , it is a good time to expand the database to include larger clusters. An argument can be made that the true nature of an element starts to become apparent at about 10 atoms $^{47,48}$. One will notice when exploring small clusters of many different types of elements, that all elements will form similar small clusters

under 5 atoms, but as the number of the atoms increases in the cluster. Special symmetries unique to each element start to appear that are unique only to that element, for example the carbon C-60 molecule is unique only to carbon. This is especially true for ground state isomers of boron, such as the boron 12 atom 
ground state structure. Thus the inclusion of clusters with up to 12 atoms of boron into the database is now appropriate. The inclusion of larger clusters will slow the code, so without a good initial guess; large amounts of computational time would be wasted if the large clusters were introduced early in the parameter fitting process. The new cluster database is shown below in figure 15 .

\begin{tabular}{|c|c|c|c|c|c|c|c|c|c|}
\hline \# & cluster & group & $\begin{array}{c}\text { Reference } \\
\text { (eVVAngstrom) }\end{array}$ & $\begin{array}{c}\text { Calculated } \\
\text { (eVV/Angstrom) }\end{array}$ & $\#$ & cluster & group & $\begin{array}{c}\begin{array}{c}\text { Reference } \\
\text { (eV/Angstrom) }\end{array} \\
\end{array}$ & $\begin{array}{c}\text { Calculated } \\
\text { (eV/Angstrom) }\end{array}$ \\
\hline \multirow{2}{*}{ B2 } & & \multirow{2}{*}{ Dih } & 0.818 & 0.701 & \multirow{4}{*}{ B7 } & & \multirow{4}{*}{$\mathrm{C} 2 \mathrm{~V}$} & 0.494 & 0.511 \\
\hline & & & -1.019721 & -1.158359 & & & & 1.654 & 1.640 \\
\hline \multirow{4}{*}{ B3 } & & \multirow{3}{*}{ D3h } & 0.890 & 0.850 & & & & $\begin{array}{l}0.809 \\
1.300\end{array}$ & $\begin{array}{l}0.810 \\
1.260\end{array}$ \\
\hline & & & \multirow{3}{*}{-2.747247} & \multirow{3}{*}{-2.803845} & & & & -3.463895 & -3.604411 \\
\hline & & & & & \multirow{4}{*}{ B7 } & & \multirow{4}{*}{$\mathrm{C} 2 \mathrm{~h}$} & 1.427 & 1.445 \\
\hline & & \multirow{4}{*}{ D4h } & & & & & & 0.781 & 0.751 \\
\hline \multirow{3}{*}{ B4 } & & & \multirow{3}{*}{-3.352603} & 1.049 & & & & $\begin{array}{l}0.797 \\
1.621\end{array}$ & $\begin{array}{l}0.829 \\
1.629\end{array}$ \\
\hline & & & & \multirow[t]{2}{*}{-3.286100} & & & & -3.405419 & -3.502480 \\
\hline & & & & & \multirow{4}{*}{ B8 } & & \multirow{4}{*}{ D8h } & 2.012 & 2.020 \\
\hline \multirow{4}{*}{ B4 } & & \multirow{4}{*}{ D2h } & $\begin{array}{l}1.193 \\
0.939\end{array}$ & $\begin{array}{l}1.142 \\
0.953\end{array}$ & & & & & \\
\hline & & & \multirow{3}{*}{-3.361147} & \multirow{3}{*}{-3.288032} & & & & -3.043245 & -3.269502 \\
\hline & & & & & & & & & \\
\hline & & & & & \multirow{4}{*}{ B9 } & & \multirow{4}{*}{ D7h } & 0.853 & 0.814 \\
\hline \multirow{4}{*}{ B5 } & & \multirow{4}{*}{$\mathrm{C} 2 \mathrm{v}$} & \multirow{4}{*}{$\begin{array}{c}0.302 \\
1.655 \\
1.549 \\
0.774 \\
-3.609799 \\
\end{array}$} & \multirow{4}{*}{$\begin{array}{c}0.380 \\
1.659 \\
1.476 \\
0.768 \\
-3.291053\end{array}$} & & & & 1.755 & 1.713 \\
\hline & & & & & & & & -3.720573 & -3.728138 \\
\hline & & & & & & & & & \\
\hline & & & & & \multirow{5}{*}{ B12 } & & \multirow{5}{*}{ C3v } & 0.968 & 0.946 \\
\hline \multirow{3}{*}{ B6 } & & \multirow{3}{*}{$\mathrm{C} 5 \mathrm{v}$} & \multirow{2}{*}{$\begin{array}{l}0.932 \\
1.365\end{array}$} & 0.998 & & & & 2.023 & 2.043 \\
\hline & & & & 1.311 & & & & $\begin{array}{l}0.430 \\
2290\end{array}$ & $\begin{array}{l}0.399 \\
2.304\end{array}$ \\
\hline & & & -3.741226 & -3.501527 & & & & 0.774 & 0.772 \\
\hline & & & 0.916 & 0.862 & & & & -4.020463 & -3.929933 \\
\hline & & & 0.220 & 0.108 & & & & 0.731 & 0.730 \\
\hline B6 & & $\mathrm{c} 2 \mathrm{~h}$ & 1.252 & 1.308 & & & & 1.618 & 1.601 \\
\hline & & C 211 & 1.452 & 1.404 & B12 & & D6d & & \\
\hline & & & $\begin{array}{c}1.507 \\
-3.585472\end{array}$ & -3.578316 & & & & & -3.989905 \\
\hline
\end{tabular}

Figure 15. The boron cluster database

At this point the code contains the complete cluster set and fictitious phases of boron. As in the previous case, copies are made and the parameter distillation process is continued. Once several parameters sets in the mid-40s 
have been obtained, it is recommended to proceed to the next stage of inclusion of extended phases of boron that have been observed and created in a laboratory.

\subsubsection{Stage 4: Inclusion of laboratory synthesized bulk phases}

We would like to have a Hamiltonian that reflects reality as much as possible. Included in the extended phase database should be at least one extended phase of boron that can be found in nature or synthesized in a laboratory that is reasonable to work with. Most if not all forms of extended boron include small 12 atom icosahedral structures of boron. Isolated, these structures are unstable but, when they are connected to other boron icosahedral structures, they form strong covalent bonds. The individual icosahedron has a triangular face ${ }^{49}$, perfect for boron's unique 3 atom electron deficient bonding nature. The alpha and beta phases of boron are the most common phases of crystalline boron synthesized. Note that it is extremely difficult to synthesize large crystals of boron and many lab samples are quite small. Boron is not found isolated in nature, but, is always found in combination with another element such as an oxide or borate. 


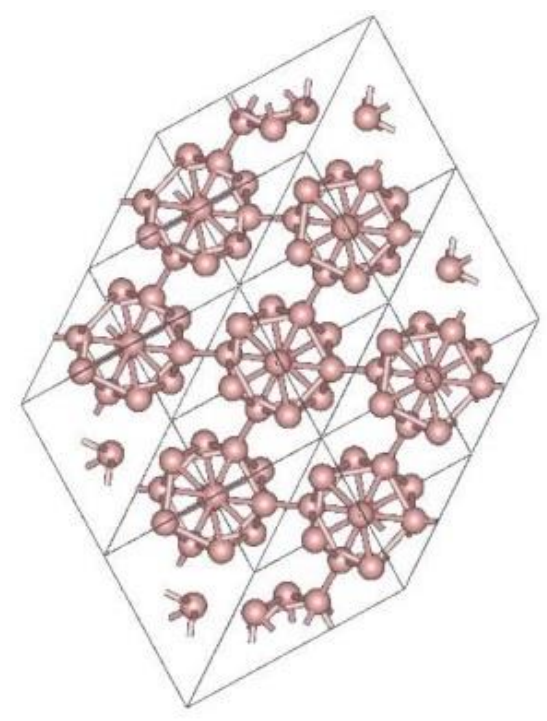

Figure 16. The alpha phase of boron

Given this, the decision was made to include the simplest crystalline form of boron found ${ }^{50}$ into the database. This is the boron alpha phase as shown in figure 16. The boron alpha phase has 12 atoms per unit cell, and indeed that unit cell is the boron icosahedron structure as discussed previously. Once the boron alpha phase is included, the same distillation process is utilized as before. The unit cell of boron alpha, although composed of icosahedrals, is rhombohedral in nature as shown in experimental image ${ }^{51}$ in figure 17 . The energy level of the alpha phase of boron is the lowest energy state in this study. The fictitious stages included are extremely high in energy. Because their energy is so high, and these phases do not represent realistic bonding scenarios found in natural boron, they are now to be excluded from the database. We now move to the next stage of fitting without fictitious phases. 


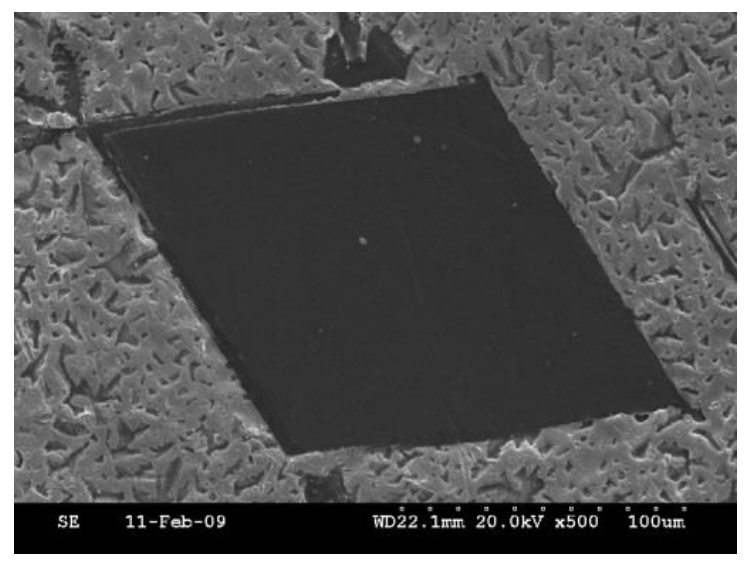

Figure 17. An image of boron alpha (rhombohedra)

\subsubsection{Stage 5: Removal of fictitious phases}

After completing stage 4, I ran a "robust check" (full detail in section 2.4. The robust check is a comparison of quality between our SCED-LCAO Hamiltonian for boron and the corresponding DFT result for various large boron systems using MD. These structures are too large to be included in the parameter fitting code, note the maximum size cluster used was 12 atoms. The robust check may include structures up to approximately one hundred atoms in size. This comparison is quite extensive, quite rigorous and slow, and is reserved as a last step in the fitting process. Once the parameter fitting codes ran for a long period time, it was noted that the fit quality progress, using the robust check comparison, was not proceeding in a rapid manner. After several months, and hundreds of attempts to gain an improvement in the quality of the boron parameters, it was concluded that by removing the very high energy (fictitious) phases of boron (i.e. sc, fcc, bcc, diamond) fitting might improve. This proved to be true during the months to come. The fictitious phases, although useful initially 
for a rough guess, were removed from the database, and only the boron alpha bulk phase was retained to represent the extended database.

Once removed, the standard method of parameter distillation proceeded, and the fit quality became more dependent upon the robust check to guide us, and less dependent upon the value of the residual in the parameter fitting code. At this point it was decided that the next stage of parameter improvement would come from increasing the quality of the ab-initio calculation used for the cluster database. This brings us to stage 6 in the fitting process.

\subsubsection{Stage 6: From density functional theory to coupled cluster theory}

In the quest to improve the database, the quality of the database was questioned. Numerous boron studies have indicated that when precision is required, DFT may be inferior to post Hartree-Fock methods ${ }^{52}$. It is always possible that the clusters or the bulk phases are poorly calculated with DFT, or they are inconsistently calculated. It is a well-known problem that even the most accurate ab-initio methods have trouble predicting the ground state structure and energies of some small clusters ${ }^{53}$.

One standard method is to calculate the optimized atom geometries using DFT (in our case B3LYP/aug-cc-PVTZ ${ }^{41,54-56}$ ) then recalculate the energy of this system with nuclear coordinates fixed (i.e. a single point calculation) using the very computationally costly coupled cluster theory ${ }^{57}$. This will give a more accurate energy calculation for the database. The system does not need to be geometry optimized because the database has no requirement for ground only 
state clusters. The cluster geometry could be slightly moved spatially from the ground state optimal configuration and we will still be accurate. The important issue is to have accurate energies for a given configuration. That configuration does not have to be the geometrically optimized minimal energy ground state for that isomer.

The $\operatorname{CCSD}(T) / a u g-c c-P V T Z^{58}$ method/basis was used to calculate the energies of the boron clusters. The energy and spatial coordinates of the bulk phase of boron alpha, calculated with DFT GGA, was left unchanged. This alteration of the database resulted in a clear decrease in the residual and the robust check greatly improved. Although the fitting has greatly improved at this point, some cluster isomers still predict the wrong energy ordering compared to other isomers with the same number of atoms. This brings us to a technique developed to help mitigate this problem. We will sacrifice fit quality of some clusters to improve fit quality of other clusters. This brings us to stage 7.

\subsubsection{Stage 7: Heavy weighting on the boron 12 cluster}

As previously mentioned, most elements start to form unique clusters around 10 atoms. No other element seems to form such shaped clusters. In the case of boron we have two interestingly shaped low lying isomers of boron 12 . The isomers are the pseudo-planar 12 atom sheet and the 12 atom double ring; they are very similar in energy, but quite different in shape. See figure 18.

The difference in energy between these two boron 12 atom isomers is very important. It was discovered that if the parameter set being tested does not 
accurately predict the proper energy ordering and difference of these two 12 atom boron isomers, the parameter set will fail to be accurate in the robust check when looking at larger cluster properties with molecular dynamics. In order to obtain the correct energy spacing and ordering, the quality of fit is judged heavily on the energy spacing and difference of these two isomers, i.e. they are heavily weighted in the fitting process. These two isomers are given extra preference in the residual calculation.
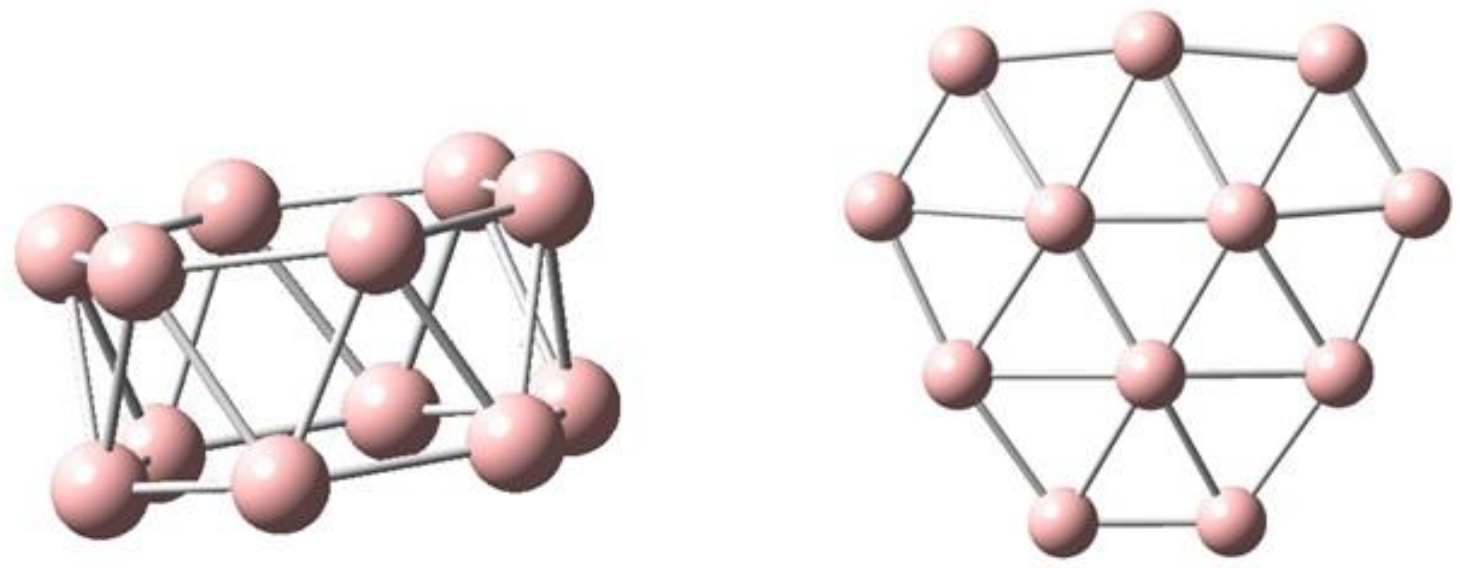

Figure 18. Two important 12 atom boron isomers

This was a crucial step in creation of the current successful boron parameter set, and is an advised trick for other semi-empirical methodologies involving other elements. Once increased weighting on these two isomers was implemented in the fitting process, the energy ordering and spacing was corrected with minimal impact to fit quality of the other clusters in the fitting set. The ability to predict the energy and spatial features of larger boron systems greatly improved. When calculating the energy ordering properties of large 
sheets of boron, a similar problem was noted. Accordingly, a similar approach was used for large boron sheets as discussed next in stage 8 .

\subsubsection{Stage 8: Addition of two dimensional extended phases}

When performing the robust check on two dimensional sheets it was noted that although initial results are quite good, there were several inconsistencies with DFT. Calculations performed on 2D sheets of boron, not only by the CMT group (we also had DFT results that were different from the SCED-LCAO results), but also by other research groups ${ }^{59,60,60-65}$ were somewhat inconsistent with the SCED-LCAO results. To mitigate this problem, two different 2 dimensional sheets were inserted into the database. One was the boron sheet made famous by the Yale group ${ }^{59}$ as shown in figure (21), which is quite low in energy when calculated using DFT, see figure (20). Note that the legends in figures (20) and (21) refer to this structure as the "removed" sheet because this structure is constructed by removing boron atoms from a sheet comprised of triangles. The other sheet inserted into the database was coined the "David" sheet, see figure (19), which is high in energy when calculated using DFT, see figure (20). 


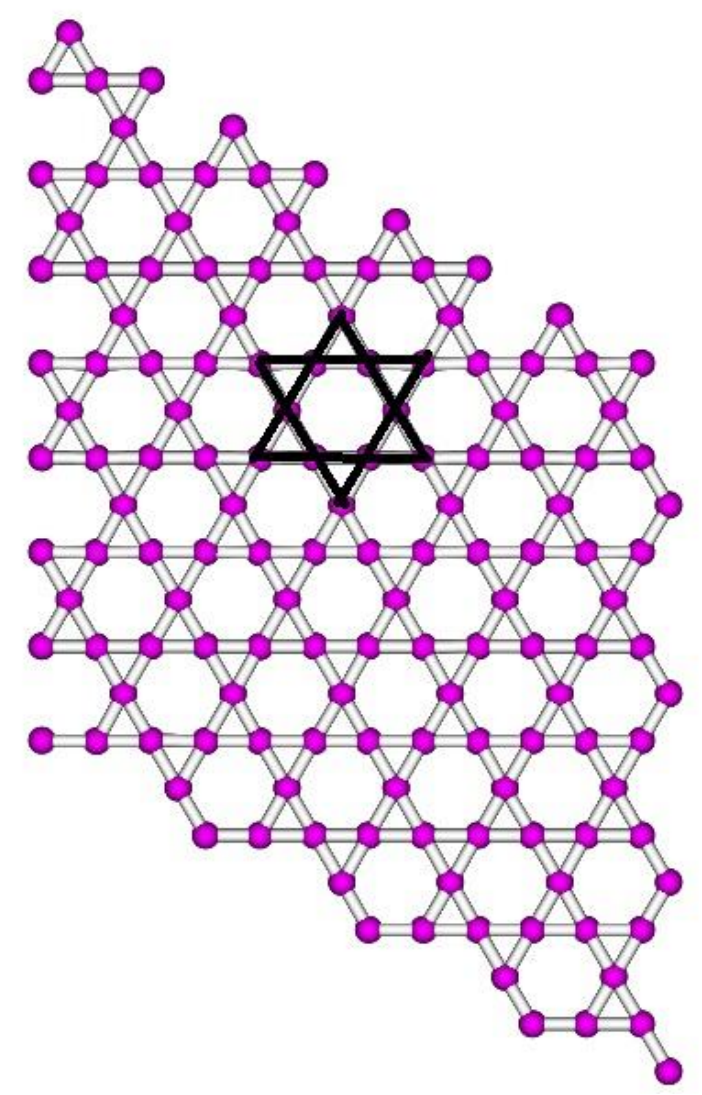

Figure 19. David sheet

The "David" sheet was accidentally discovered during a reconstruction simulation of a hexagonal sheet, and it was named "David" because the unit cell resembles the Star of David ${ }^{66}$. At first the sheet was thought to possess a low energy, but, after simulating the sheet in DFT it was determined there was an energy ordering problem with the boron parameters. The "David" sheet was stable in DFT and in SCED-LCAO when heated and cooled slightly. 


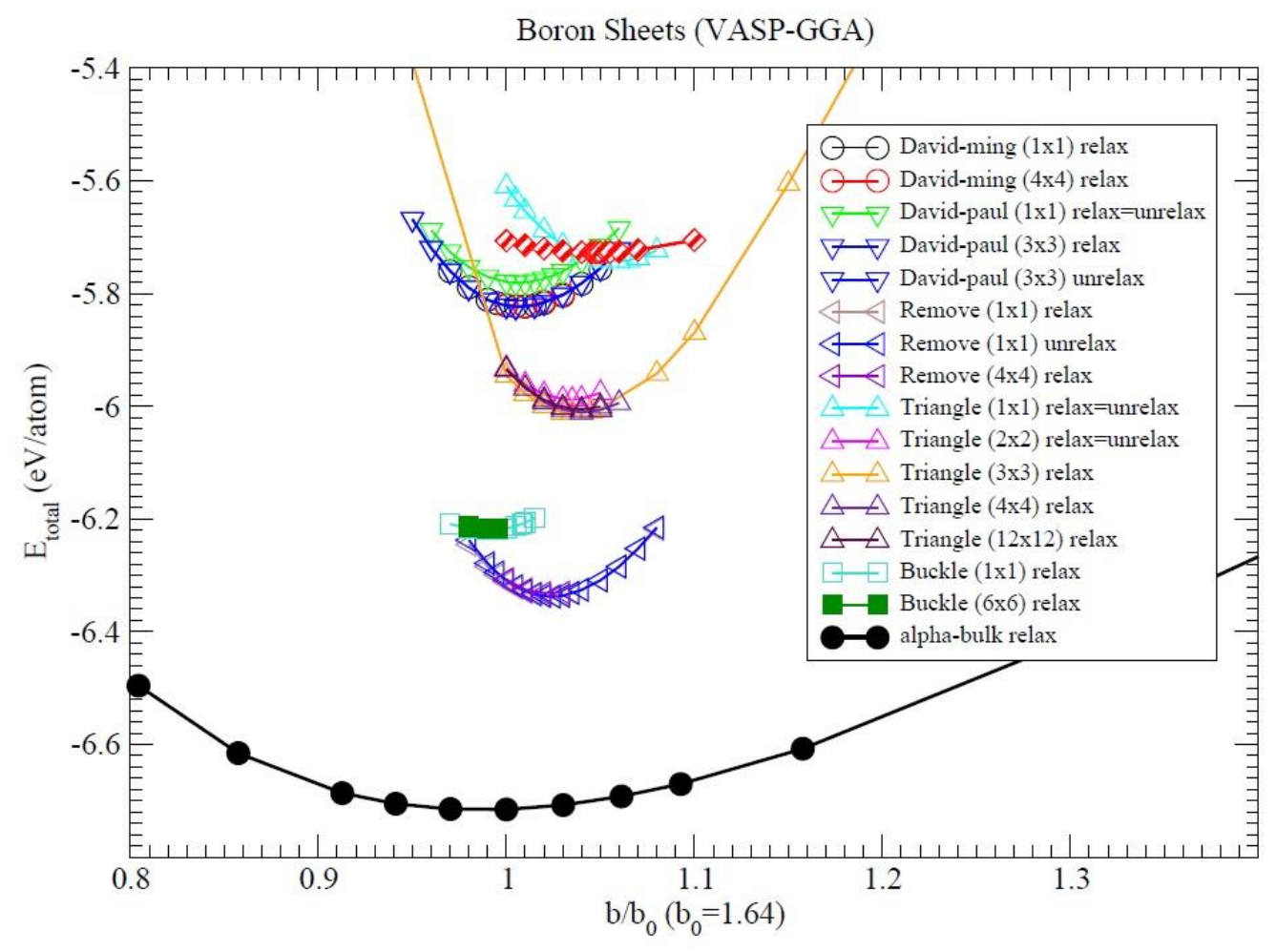

Figure 20. DFT results of 2D boron sheets (Yu Ming)

The ordering problem was solved by including the Yale (i.e. removed) sheet and the "David" sheet in the fitting code. After refitting, the parameter fit improved greatly and energy ordering for the sheets was corrected. The bulk (i.e. extended) database now includes the alpha boron phase (3D) and two sheets of boron (2D). The fit quality is as follows: 


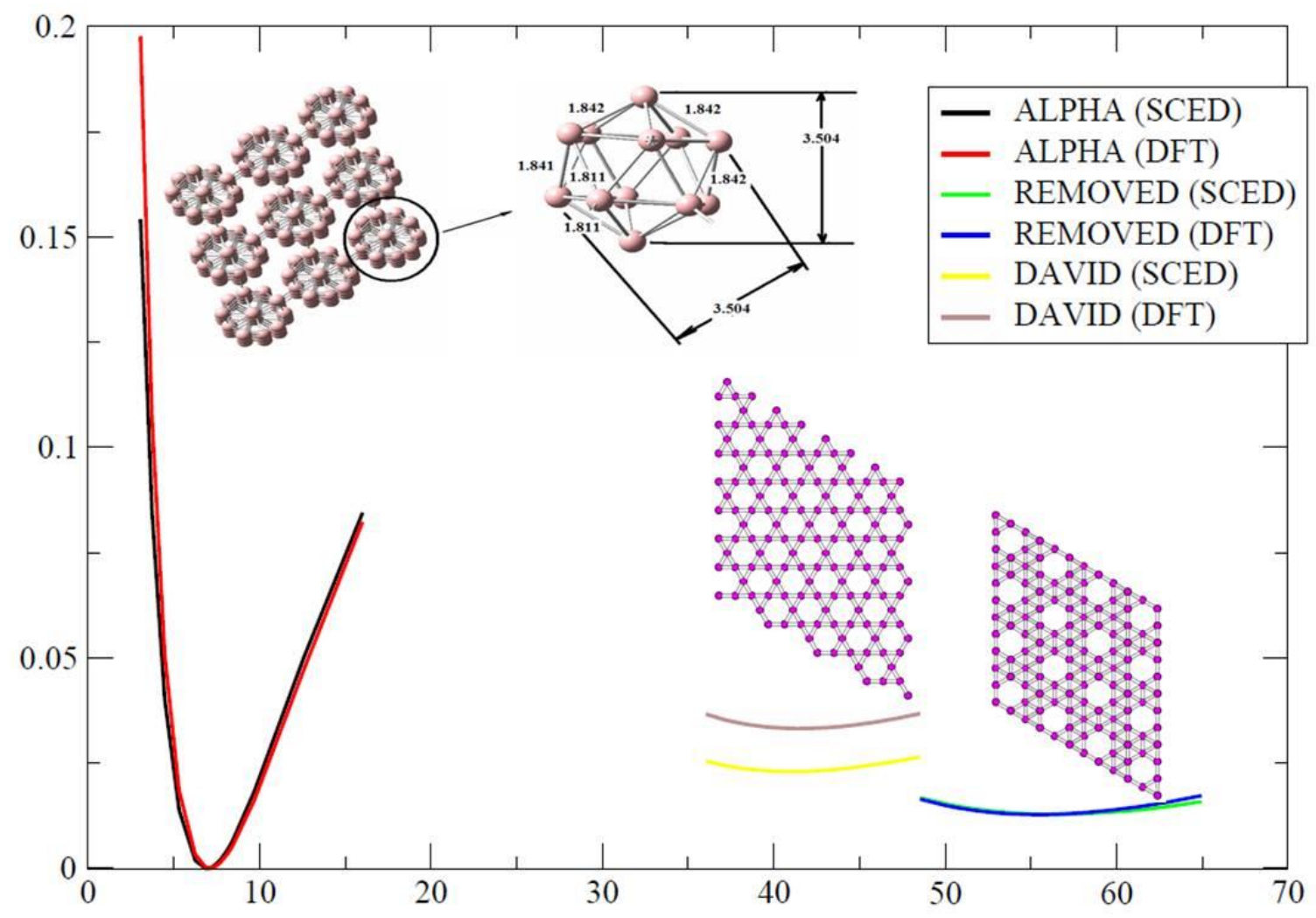

Figure 21. Fit quality of extended phases

It is worth noting that the fit for the Yale sheet and the fit for boron alpha are quite good. The shape of the fit for the David sheet is good but the energy prediction is off by approximately $0.01 \mathrm{eV} /$ atoms, due to the David sheet being such a high energy sheet. In this case the shape and location of the minimum were more important.

\subsubsection{Conclusions about fitting}

We have now concluded the 8 stage fitting process. We now have a very good set of boron parameters, but they are not perfect and never will be. The very nature of semi-empirical methods is not to find a perfect set of parameters 
for a given element, but to find a sufficient set of parameters in a finite period of time that can be used to rapidly calculate properties of the system:

\begin{tabular}{|c|c|c|c|c|c|c|c|}
\hline $\begin{array}{l}\text { Hamiltonian } \\
\text { Parameters }\end{array}$ & BORON & \begin{tabular}{|l|} 
Hamiltonian \\
Parameters
\end{tabular} & BORON & $\begin{array}{c}\text { Overlap } \\
\text { Parameters }\end{array}$ & BORON & $\begin{array}{c}\text { Overlap } \\
\text { Parameters }\end{array}$ & BORON \\
\hline$\varepsilon_{s}$ & -13.46000000 & $U$ & 18.58616964 & $\alpha_{p p \pi}$ & 1.42536416 & $B_{s p \sigma}$ & 0.46586279 \\
\hline$\varepsilon_{p}$ & -8.43000000 & $\alpha_{K}$ & 0.17264701 & $\alpha_{p p \sigma}$ & 3.63410778 & $B_{s s \sigma}$ & 0.31792180 \\
\hline$\varepsilon_{s}^{\prime}$ & -16.41094650 & $A_{N}$ & -2.07510516 & $\alpha_{s p \sigma}$ & 1.81974984 & $d_{p p \pi}$ & 0.32644951 \\
\hline$\varepsilon_{p}^{\prime}$ & -14.52914060 & $B_{N}$ & -1.14305936 & $\alpha_{s s \sigma}$ & 1.47650365 & $d_{p p \sigma}$ & 1.52882755 \\
\hline$W_{p}^{0}$ & 0.18256068 & $\alpha_{N}$ & 2.50226452 & $B_{p p \pi}$ & -0.30545438 & $d_{s p \sigma}$ & 1.11849315 \\
\hline$W^{0}{ }_{s}$ & -0.92058150 & $d_{N}$ & -0.59707673 & $B_{p p \sigma}$ & -0.90613327 & $d_{s s \sigma}$ & 0.52011830 \\
\hline$\alpha_{p, w}$ & 1.22514015 & $B_{Z}$ & 2.91681729 & & & & \\
\hline$\alpha_{s, w}$ & 2.17182985 & & & & & & \\
\hline
\end{tabular}

Figure 22. Final boron parameters

There will always be some level of non-transferability, and there will always be some question of the accuracy of the calculation given any set of parameters. But, this is also true when changing different types of exchange/correlation functionals in DFT. DFT is far slower and far more memory intense than the SCED-LCAO semi-empirical method. The final optimized set of boron parameters found using the 8 stage process described above are shown in figure 22

This set of parameters represents a very long evolution of work over many years. If these parameters are truncated, unreliable results may occur. This is because the system is extremely nonlinear and sensitive to small changes in the 
parameters. Note that $\varepsilon_{s}$ and $\varepsilon_{p}$ are truncated, this is because they were fixed throughout the total fitting process.

\subsection{MOLECULAR DYNAMICS ROBUSTNESS CHECK}

\subsubsection{Robust check \#1: 6 clusters of interest}

A secondary database is utilized to perform a robustness check. This database is not included in the parameter fitting code because this would be far too computationally costly to include. The robust check database contains extremely large clusters, extended phases and utilizes a MD routine written by the University of Louisville Condensed Matter Theory Group ${ }^{16}$. Once many sets of parameters have been chosen as candidates from the fitting code they are tested in the MD code to find the best set of parameters. This is the final step in the parameter decision making process. The approach starts with large clusters and extended phases calculated in DFT. The coordinates of those structures are input into the SCED-LCAO Hamiltonian in the MD code. In most cases if the structure changes drastically as the system is allowed to relax, that set of parameters is discarded and a different set is chosen. The only exception is when we would expect reconstruction to a different structure. The test cases for the robust check are shown in figure 23. 


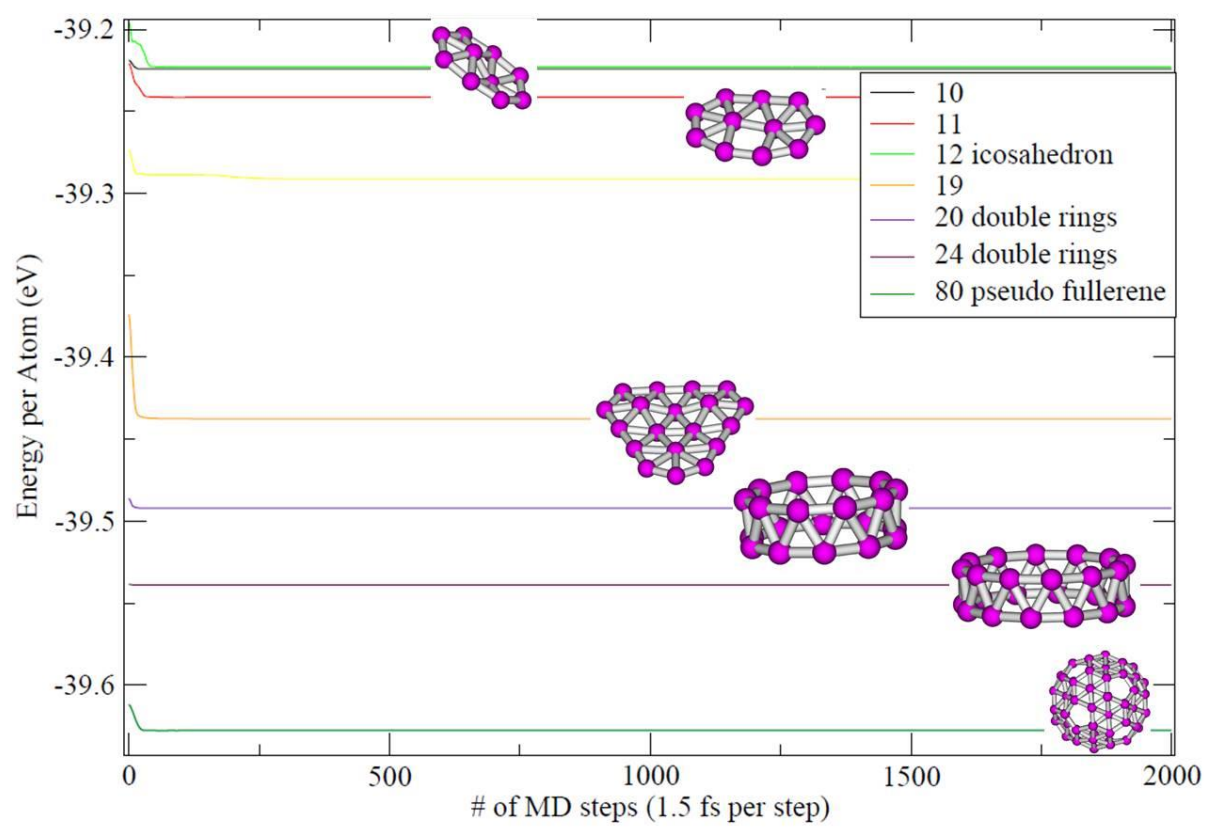

Figure 23. First of three robust checks

The robust check includes structures that are not included in the fitting database, are generally larger structures, and have been previously been studied using $\mathrm{DFT}^{34,39,40,52,67-72}$. The energy ordering is checked and the spatial structure is checked. In figure 23 we see ground state structures of boron $10,11,12$ (icosahedral), 19, 20, 24 and we have the boron 80 quasi-fullerene structure. The plot shown in figure 23 shows a successful plot because every curve is flat. If we feed in the coordinates from the output of our DFT simulations we should get approximately the same structure back out from the MD code using the SCEDLCAO Hamiltonian (i.e. the curve should be flat with no reconstruction of the structure). Also, energy ordering compared to DFT is correct. This would be considered a successful robustness check, and the parameter set would be considered a strong candidate for the next stage of robust checking. 


\subsubsection{Robust check \#2: three isomers of the 13 atom boron}

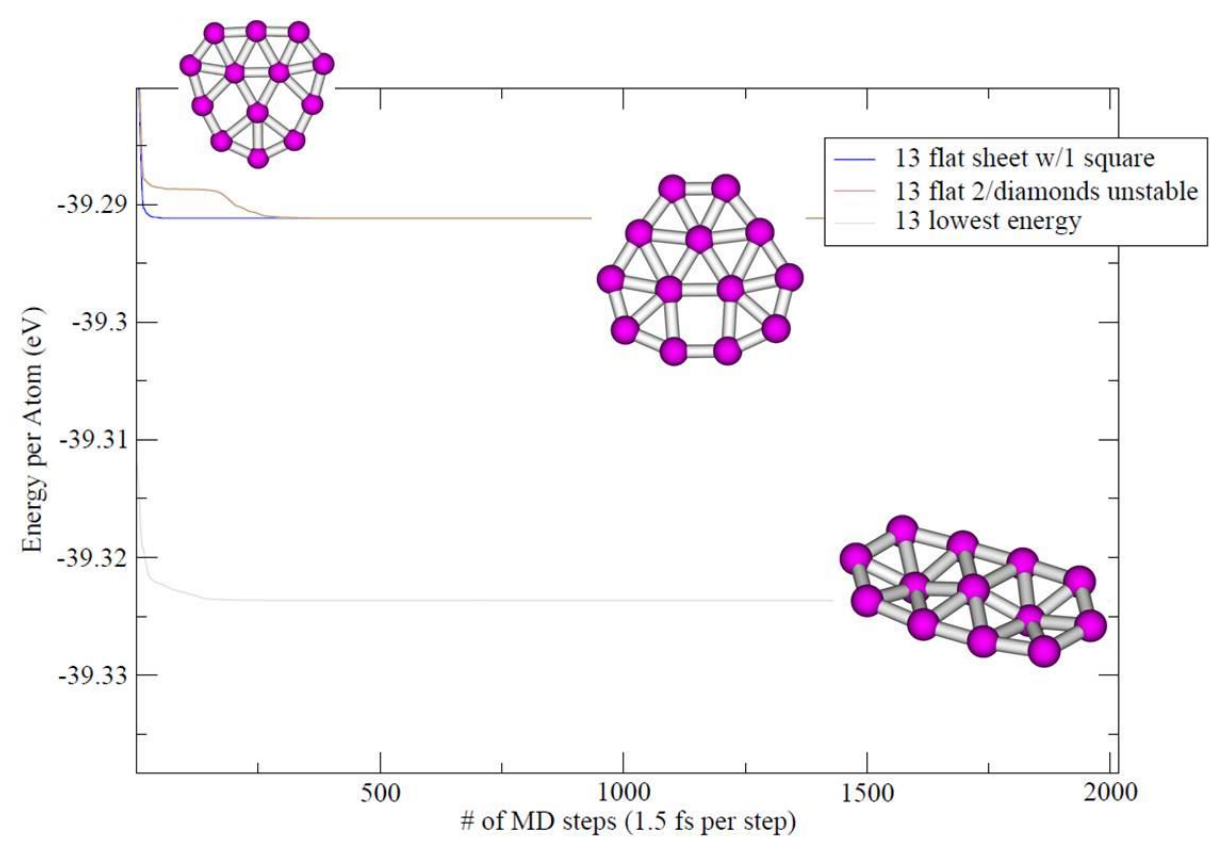

Figure 24. Three isomers of 13 atom boron

The second robust check tests the ability to correctly order and reconstruct 3 isomers of 13 atoms boron. The highest energy boron isomer is similar in energy to the next highest energy isomer as shown in figure 24 . The highest energy isomer reconstructs into the second highest energy isomer within the MD code. Energy ordering before reconstruction is consistent with DFT results. This is a very promising result. The lowest energy isomer is much lower than the other two, and this is also consistent with DFT results. The above plot is very consistent with DFT, and would be considered a more stringent result than robust check \#1. This plot would be considered a pass, and the parameter set would be moved on to robust check \#3. 


\subsubsection{Robust check \#3: two isomers of the 15 atom boron}

Robust check \#3 is a comparison of energy ordering and structure stability of two 15 atom isomers of boron as shown in figure 25 .

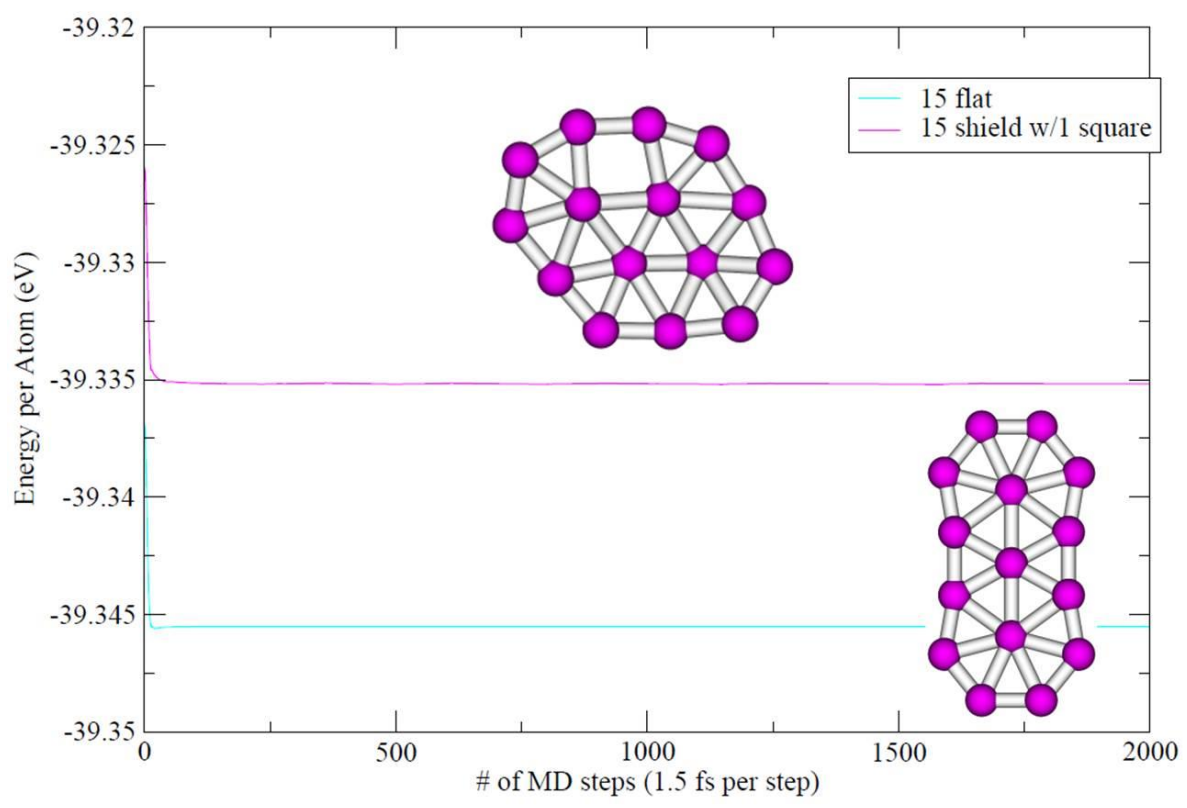

Figure 25. Two isomers of 15 atom boron

It is noted that achieving a successful set of parameters that meets all three robust checks was exceedingly difficult. This will end the robust check on larger clusters. We will now check sheet stability.

\subsubsection{Robust check \#4: reconstruction of the triangular to buckled sheet}

Within the framework of DFT a triangular sheet of boron is a high energy structure. This structure will spontaneously reconstruct to buckled sheet of triangles in perfect rows. The current robust check will test this reconstruction. If the reconstruction is not a perfect reconstruction from a flat triangular sheet to a perfectly buckled sheet with perfect rows, the set the set of parameters is 
discarded. I stress perfectly because many parameters sets would buckle, but not all were perfectly buckled.

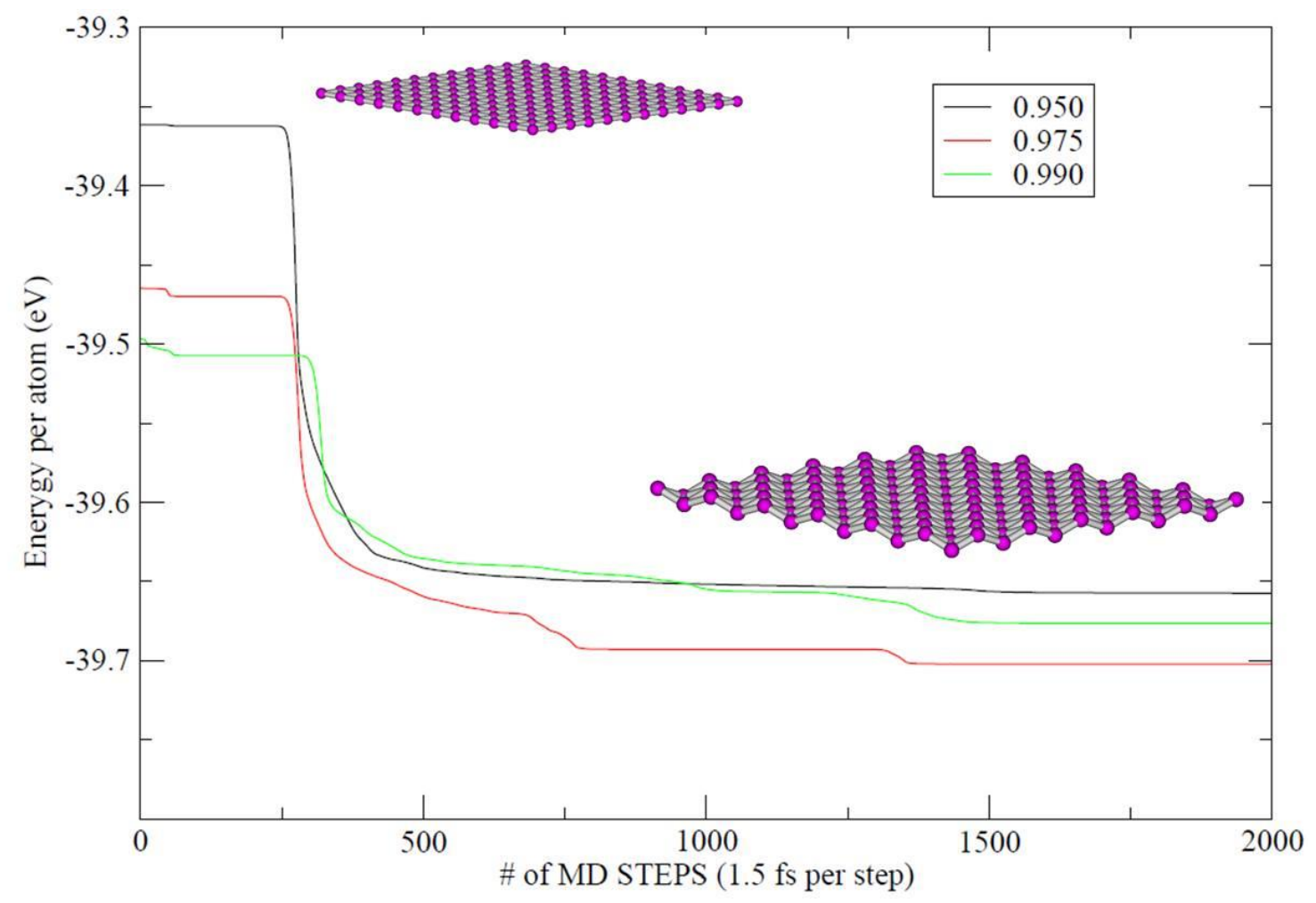

Figure 26. Reconstruction of the triangular to buckled sheet

In figure 26 three initial triangular sheets of boron with varying lattice constants are allowed to reconstruct in the MD code, using the SCED-LCAO method. The final lowest energy structure is a perfectly buckled sheet of triangles in perfect rows. This is exactly the result that was sought. If the parameter set could achieve this reconstruction it was considered a strong candidate for the next robust check. Note that if the structure is pre-stretched it may crumble when the system starts to relax, or it will not reconstruct perfectly. 


\subsubsection{Robust check \#5: Energy ordering of all sheets and conclusions}

The absolute final robust check is energy ordering of the triangular, "David", buckled, and Yale (i.e. removed) sheets.

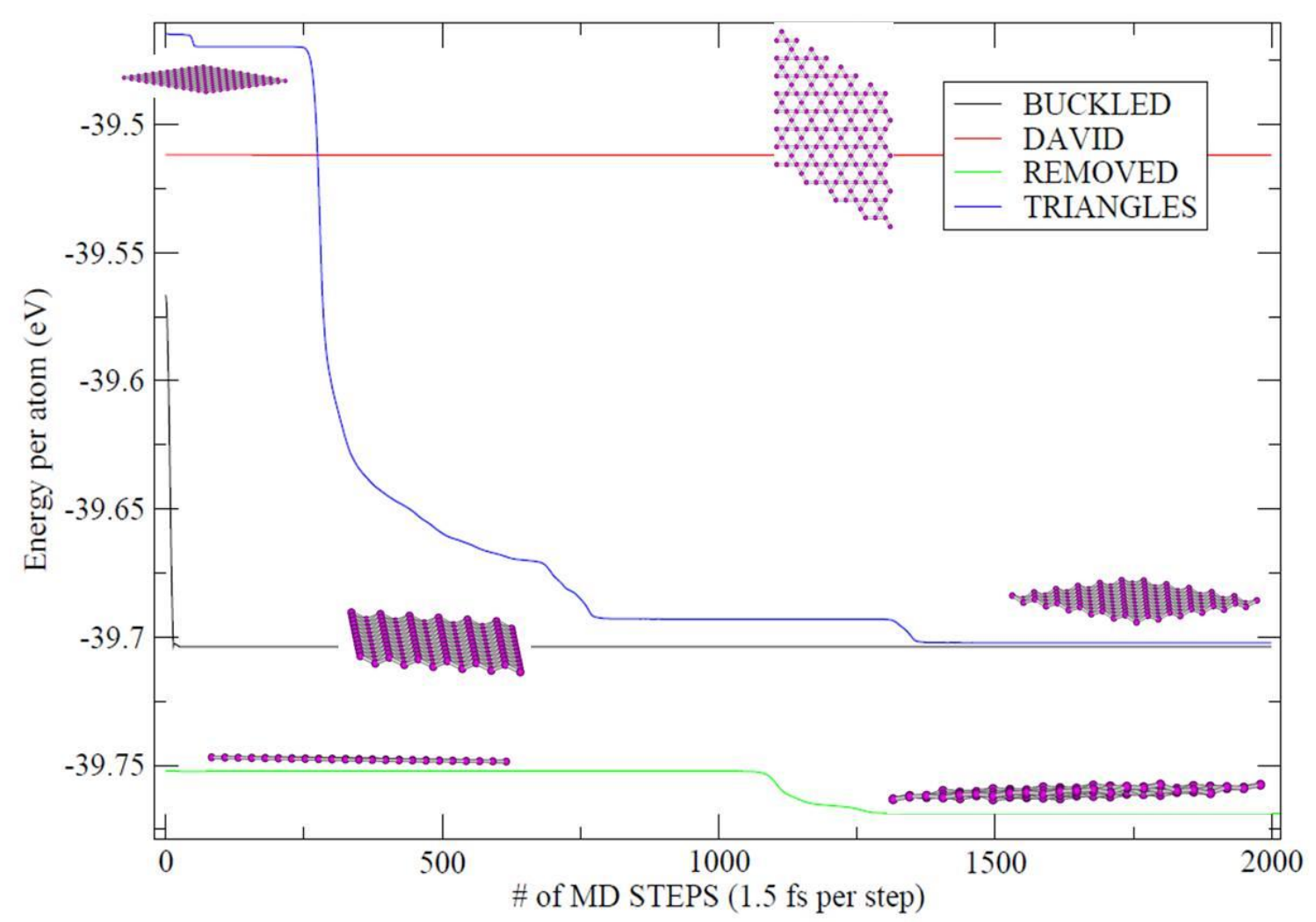

Figure 27. Energy ordering of all examined boron sheets

If a parameter set could pass all stages of the fitting checks previously discussed and all stages of the robust checks mentioned above, only then is the boron parameter set that is being tested considered a high enough quality to have the ability to predict the behavior of boron as compared to DFT methods. The above plot meets full consistency with DFT with one minor caveat. If you look closely at the Yale (i.e. removed) sheet, it reconstructs to a slightly lower energy structure. This structure is about $0.04 \mathrm{eV} /$ atom lower than the initial 
relaxed structure. Also, this structure had to run for $1000 \mathrm{MD}$ steps before it reconstructed, a feature that many may miss if they did not run the simulation long enough. This may actually represent on some level the accuracy of the SCED-LCAO Hamiltonian for this given set of parameters as applied to boron sheets. It is somewhat amazing that this set of parameters met so many checks and balances, and yet slightly gets this one structure slightly wrong. In the quest to correct this minor flaw, after a month of work, I decided that I cannot meet all stage checks and robust checks and correct this in any finite period of time. Initially it was stated in this project that a semi-empirical method will never agree with any ab-initio method in every case for every system and element. But, after eight fitting stages and five robust checks, I have to assume my error is 0.04 eV/atom for boron sheets. This is the error of my parameter set used with this particular Hamiltonian. It may be assumed that for some structures it may not be possible to distinguish the difference in energy for energies smaller than 0.04 eV/atom. When I get to these small differences in energy levels, reliability may come into question. Alternatively, it is possible that there is something odd about the sheet. But, is more likely that for this particular system predictive power is lower, but we cannot say this true for any other system not tested. In fact it is sufficiently predictive for most systems as evidenced by its success as shown above with all other systems tested.

We are now in a position to use our Hamiltonian. The next chapter will outline several applications. 


\section{CHAPTER III: BORON SIMULATIONS}

In the previous chapter we developed a fitting methodology to fabricate an accurate semi-empirical Hamiltonian for boron. The boron Hamiltonian was thoroughly tested and found to be extremely accurate. We will now proceed with several applications of the boron Hamiltonian of interest.

\subsection{TWO DIMENSIONAL SHEETS}

Although two dimensional boron sheets were used in the fitting process, only certain properties of the sheet under test were used as criteria for the fitting. All other properties not considered in the fitting process are considered predictions. This is also true for every boron structure considered during the fitting process. For example, if the energy of a cluster was included in the fitting process, but not a bond angle in that cluster, then the energy is a fitting property and the bond angle is a prediction. The full results of the boron sheets simulation are summarized in the previous section (2.4.5, figure 27). The results are not only a robust check, but also a prediction.

\subsection{THE BORON 80 ATOM FULLERENE STRUCTURE}

A large amount of focus was spent on the boron 80 buckyball several years $\mathrm{ago}^{73}$. The initial result indicated the structure the structure is stable, but, I had found an 80 atom fullerene structure that was a bit lower than the B80 
buckyball, and I noticed the structure was not actually an Ih structure; it was actually Th and maybe C2h symmetry ${ }^{39}$. This result was found with an earlier set of boron parameters. Another 80 atom structure was found, it was amorphous in nature but was significantly lower in energy than the B80 buckyball.

Since then several events have occurred:

1. A lab in Leuven ${ }^{74}$ asserted that the structure is not lh but is Th.

2. A correction paper by Boris I. Yakobson was released to Physical Review stating that indeed the symmetry was not $\mathrm{lh}$ as originally thought ${ }^{75}$.

3. Another lab discovered a lower energy $B 80$ state $^{76}$ than the $B 80$ fullerene.

4. It was theorized that stuffed boron fullerenes have lower energy than unstuffed ${ }^{77}$.

5. I made my assertion in a poster presentation at an APS March meeting ${ }^{39}$ that the $\mathrm{B} 80$ cluster was not stable, was not Ih, and was not the lowest energy isomer for 80 atoms of boron. At that time I was still not confident about the current boron Hamiltonian. Since there are now confirmation my assertions, it must be concluded that the boron SCED-LCAO Hamiltonian has surprising predictive power.

The lowest energy state found with the current SCED-LCAO Hamiltonian was a Ch symmetry state with some atoms pointing out and others pointing in. 
The differences in energy of these structures are exceedingly small, $0.004 \mathrm{eV} /$ atom. This is fairly consistent with small basis set calculations in DFT. The final relaxed structure originating from an Ih structure is shown below in figure 28. It is noted that central 20 atoms are quite unstable when the system is heated and starts oscillating. These central atoms eventually break the symmetry of the structure during the heating process and holes form in the structure. Once a hole is formed the structure will not heal according to every simulation attempted. If heated a bit more the whole fullerene collapses into an amorphous structure, when cooled it stays amorphous.

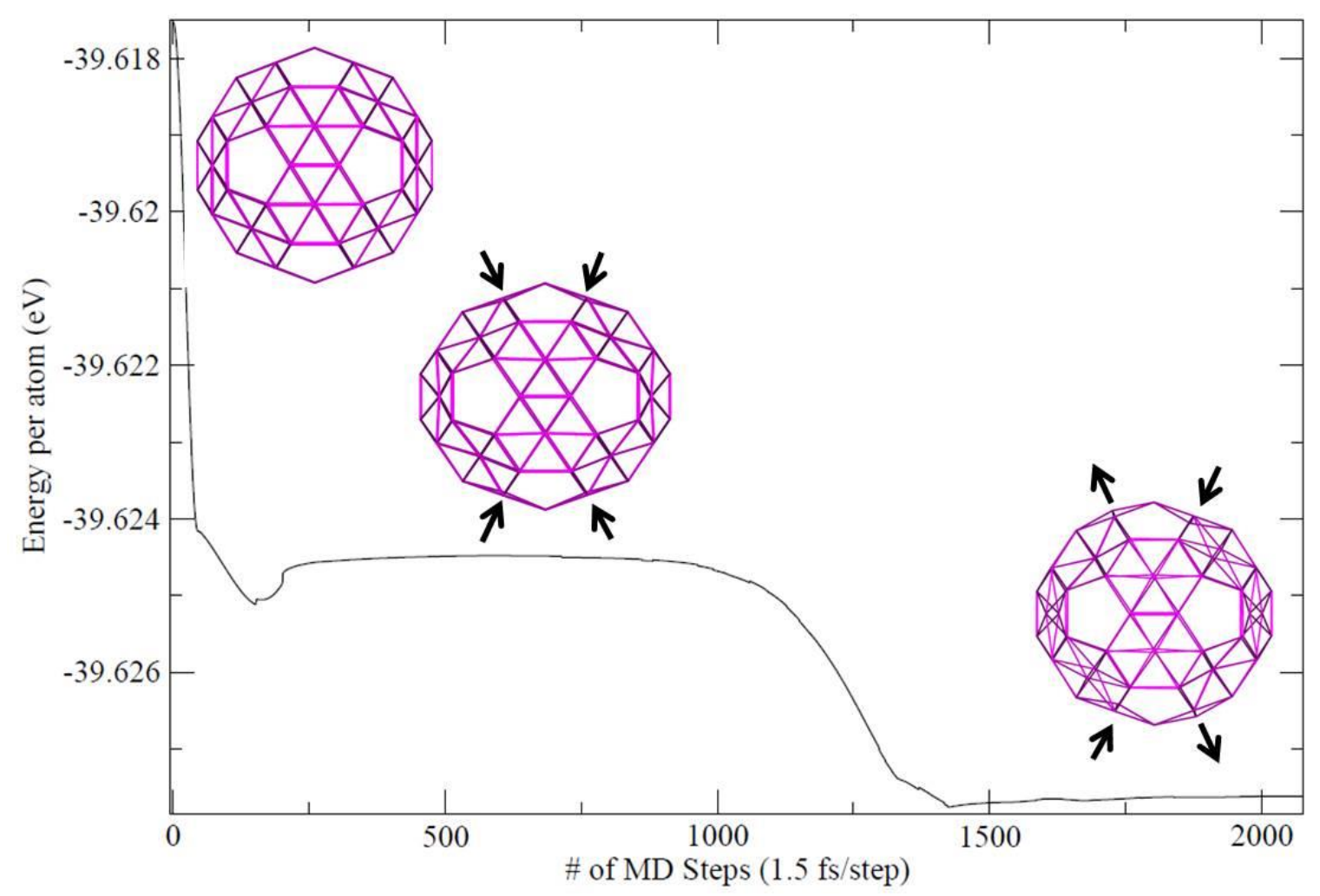

Figure 28. The B80 boron Ih, Th, and C2h fullerene from left to right. 
The final relaxed B80 structure is a semiconductor with a homo lumo gap of $0.92 \mathrm{eV}$. This is not that different than silicon, but, this structure will most likely not be found in nature for numerous reasons, and has still not been found many years later. Density of states and homo lumo gap of the B80 cluster are shown in figure 29 .

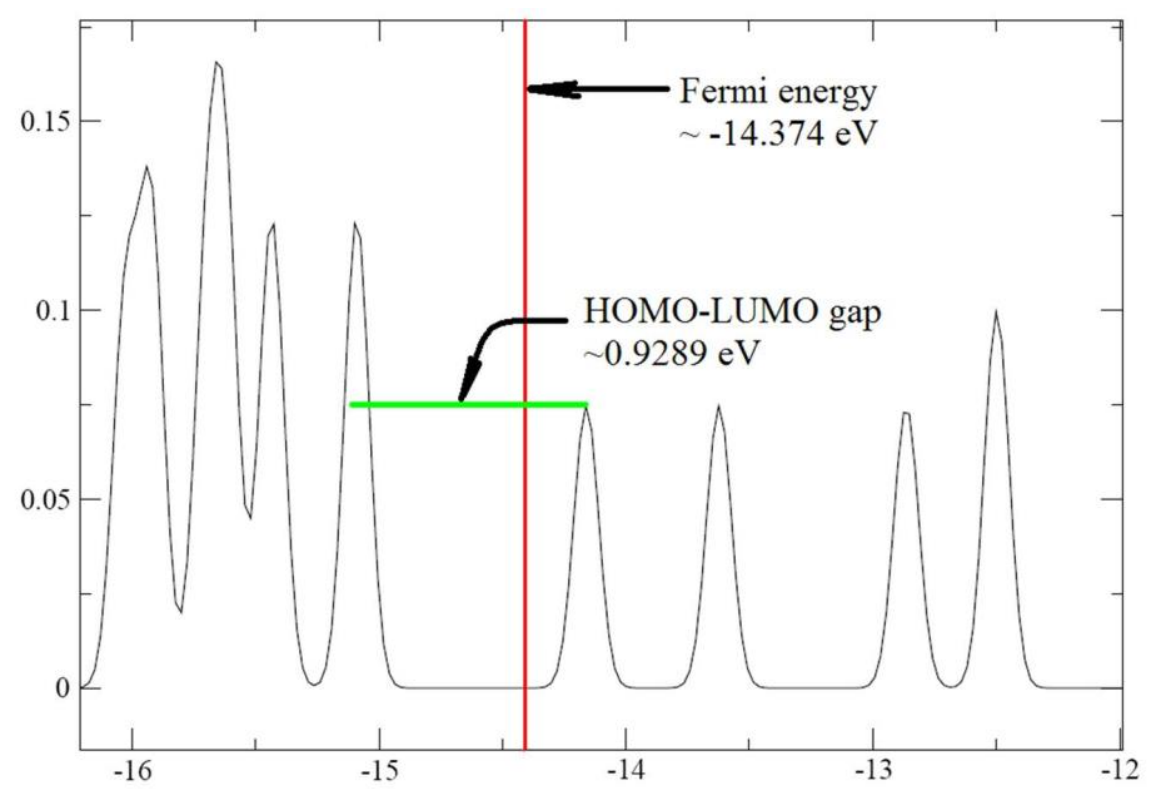

Figure 29. Density of states plot and homo lumo gap

\subsection{RANDOM BORON CLUSTERS}

One early application, using an earlier (yet heavily tested) set of boron parameters, of the boron Hamiltonian was the comparison of random boron structures that are fully amorphous, with select icosahedral centered cuts (i.e. vertex centered, see section 3.4.5), in between icosahedral centered cuts (i.e. body centered, see section 3.4.5) and the B80 buckyball (see section 3.2). The random structures, see figure 31 , were created using a random number 
generator then power quenched ${ }^{78,79}(P Q)$ using MD. Once power quenched, the clusters were heated to a moderate temperature, allowed to vibrate and move using simulated annealing (SA) then finally quenched again. The results are quite remarkable, see figure 30 .

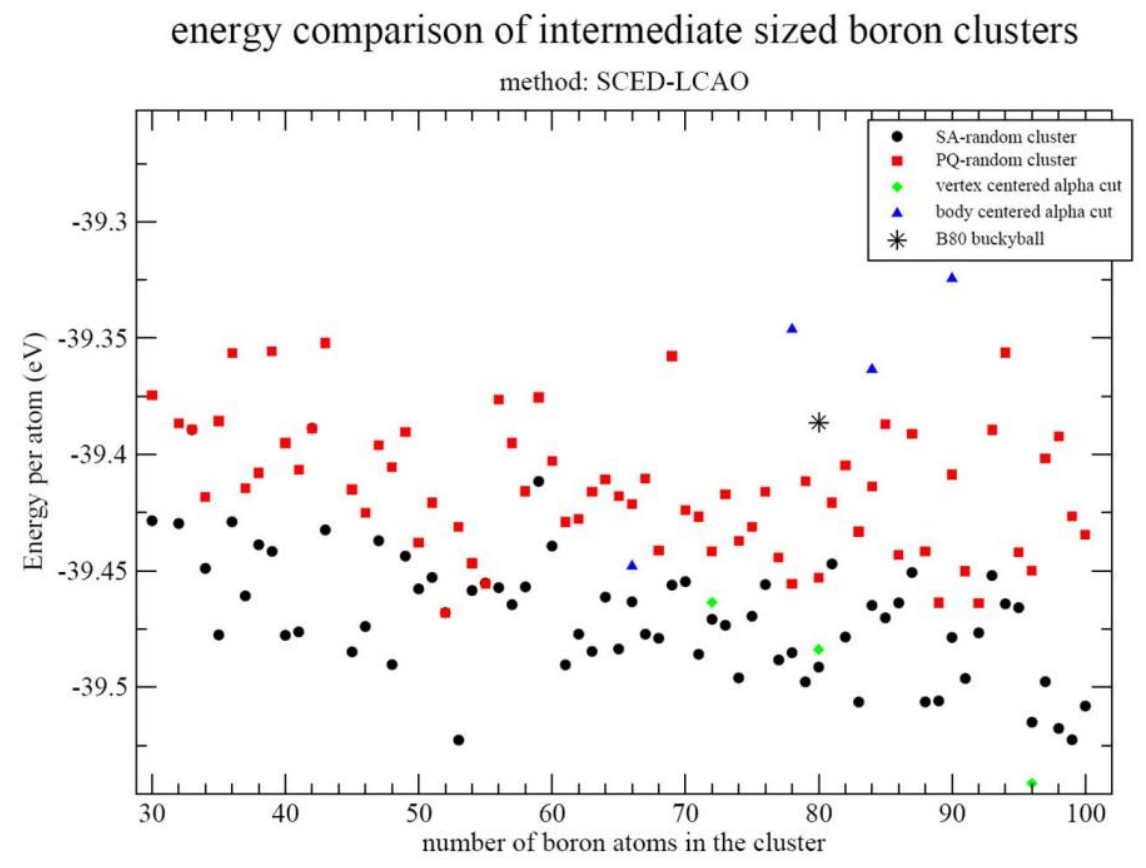

Figure 30. Random clusters vs. cuts and B80

The finding demonstrates that most amorphous clusters between 30 and 100 atoms, that are fairly low in energy, are lower in energy per atom than the boron 80 buckyball $^{70,80,81}$. 


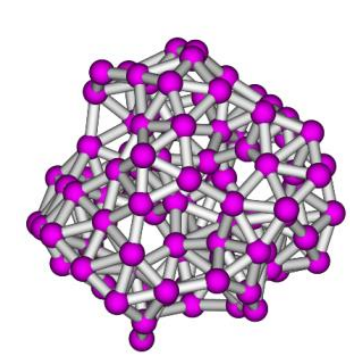

101 boron atoms

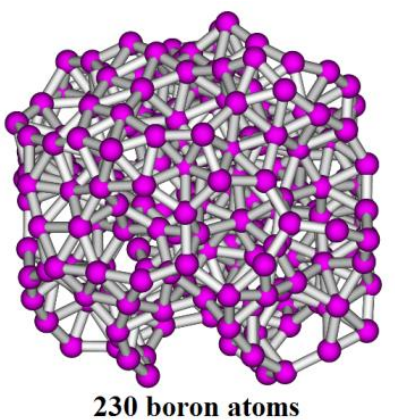

Typical random quenched amorphous boron cluster

Figure 31. Typical amorphous random boron cluster

This does not mean that the boron 80 buckyball cannot exist. But, it does imply that any laboratory fabrication technique, for boron cluster construction, that involves significant heat; the symmetric structures that could be produced will have to compete with boron's natural tendency to enjoy being in an amorphous state.

\subsection{ALPHA BORON CUT CLUSTERS: RHOMBOHEDRAL SYMMETRY}

Most boron structures have either icosahedral ${ }^{37}$ structures within, or they are amorphous ${ }^{82}$ in structure. It is of interest to examine some of these structures, in particular the icosahedral based structures. Beta boron seems to be the current lowest energy ${ }^{83-85}$ phase of boron. Beta boron is also the hardest and highest temperature phase of boron and it coexists at room temperature with alpha boron. They are competitive in energy, with beta boron being only slightly lower in the ground state energy than alpha boron. The extended phase of alpha 
boron, infinite in size, was found to have an energy corresponding to approximately $-40.12 \mathrm{eV}$ using the SCED-LCAO Hamiltonian.

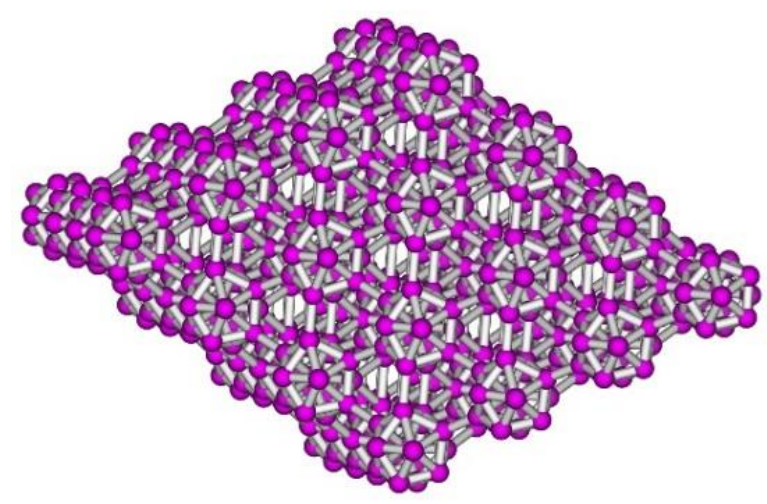

Figure 32. A 4_4_4 slab of boron alpha

Small pieces of alpha boron may make good candidates for small nanostructures. In the following sections the boron Hamiltonian is used to predict properties of small alpha boron cut clusters with rhombohedral symmetry.

Every rhombohedral cut is tagged with a label such as $2 \_3 \_4$ or $5 \_3 \_2$, i.e., $\mathrm{i} \_$_k where the indices designate how many icosahedra there are in the cluster. The total number of atoms in the cluster is $12^{*} i^{*} j^{*} k$. Figure 32 is designated 4_4_4, for example. A sheet of icosahedra would be designated ij_1 (or $\mathrm{i} \_$), because it is one layer thick. A chain of $\mathrm{i}$ icosahedra would be designated i_1_1 (or i), because it is of unit thickness and width.

\subsubsection{D Chains of Icosahedra}

One of the highest energy structures I have simulated is the chain of $\mathrm{i}$ (i_1_1) boron icosahedra as shown in figure 36. The finite chain, like all other 
structures comprised of only boron icosahedra, has vanishing homo-lumo gap, and are metallic in nature.

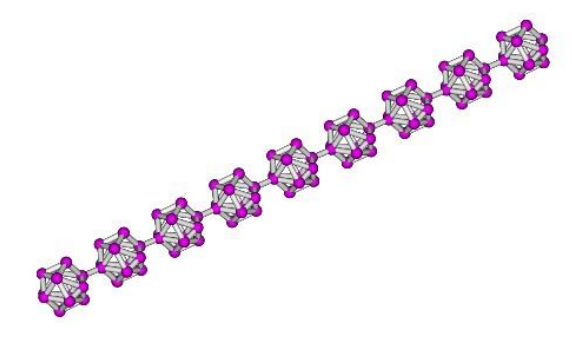

Figure 33. The icosahedral boron 1D chain

The chain is considered to be a rhombohedral cut of boron. During the finite temperature simulations, winding of the chain was not observed. It was observed that the bonds break between the icosahedra and the chain breaks into pieces, and self-consistency cannot be achieved. An energy plot of chain length for up to 300 boron atoms is shown in figure 34 .

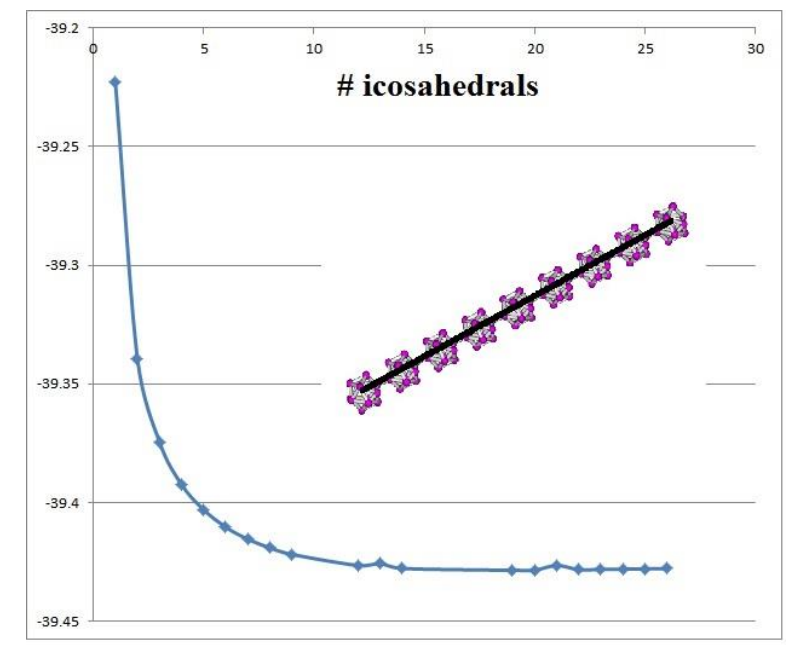

Figure 34. Energy plot of an icosahedral 1D chain as a function of length. 
When the number of icosahedra gets to about 10, the energy per atom is approximately constant. These chains are very high energy structures and thus might be difficult to fabricate.

\subsubsection{D sheets of icosahedra}

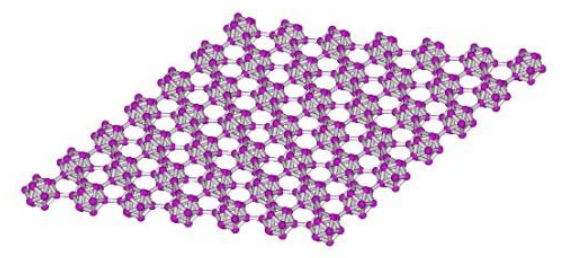

Figure 35. The icosahedra sheet

If one can imagine a sheet that is completely made out of icosahedra as seen in figure 35 , it would have a quasi-2D structure. If we view the icosahedra as single entities, one can imagine flat sheets of this nature. The sheet size would be designated as $i \_j$. If we calculate the energy of many such sheets we see a smooth trend as shown in figure 36 . The sheets with $i=j$ have the lowest energy if the total number of atoms is fixed. For example a 5_20 sheet is higher in energy then a 10_10 sheet even though they have the same number of atoms. The icosahedral sheets have no homo-lumo gap, for example the homo-lumo gap of the $7 \_7$ sheet is $0.0048 \mathrm{eV}$. Sheet energies/atom are also fairly high, especially if they are more strip in nature. 

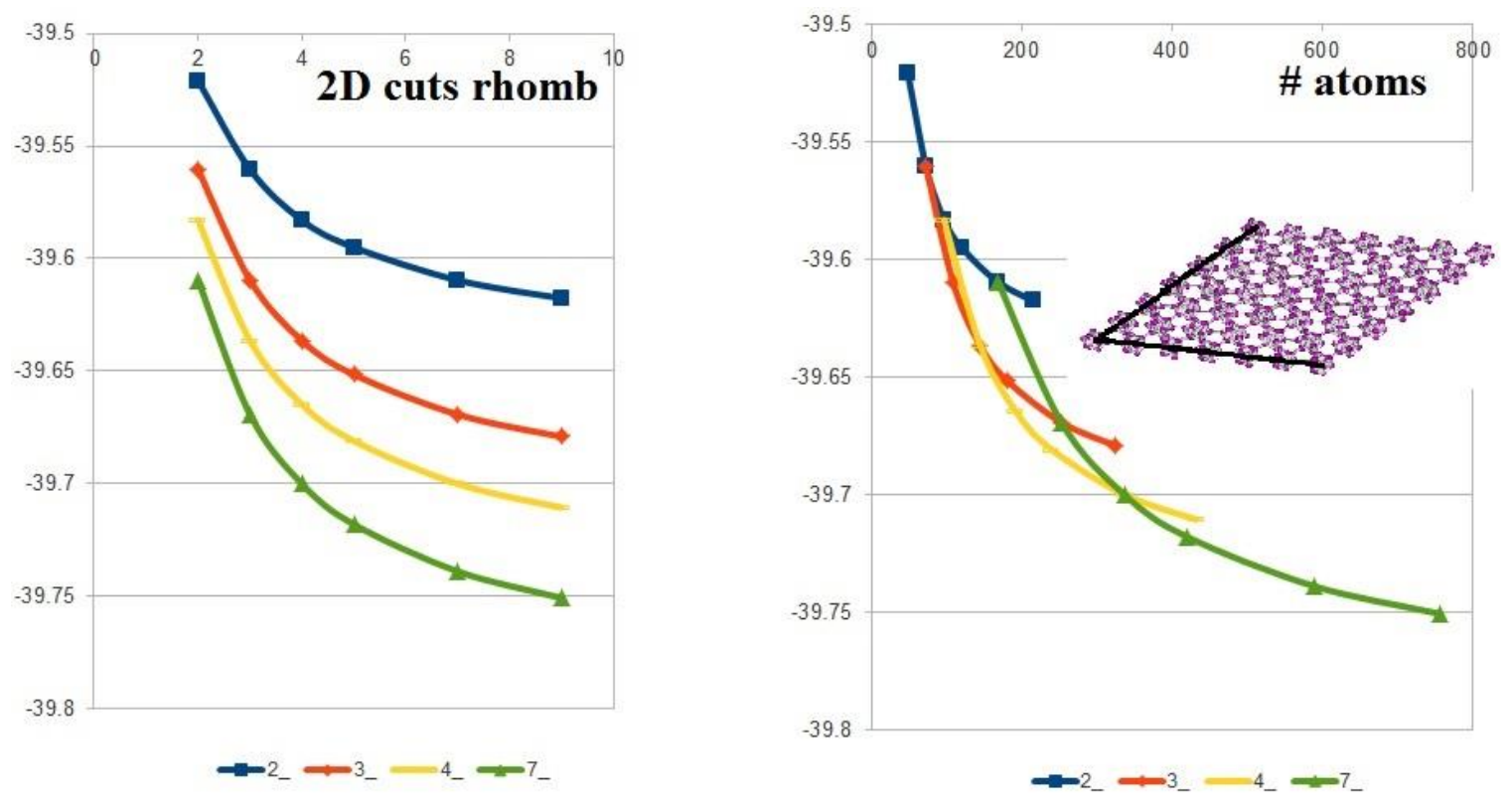

Figure 36. Energy plot of 2D icosahedral sheets

\subsubsection{D structures of icosahedra}

Compact 3D rhombohedral cut structures are quite low in energy. A plot of various energies for select clusters is shown in figure 37 . The index indicates the number of icosahedra. A chart of select homo-lumo gaps, all are metallic, is shown in figure 38. 3D structures keep decreasing in energy as they grow in volume as long as they grow homogeneously in all directions. Compact structures tend to level out in energy at the same value as bulk boron alpha as previously mentioned at sizes above 50,000 atoms. 
Energy/atom for various $\mathbf{i} \mathbf{j}_{-} \mathbf{k}$

rhombohedral 3D boron alpha cuts

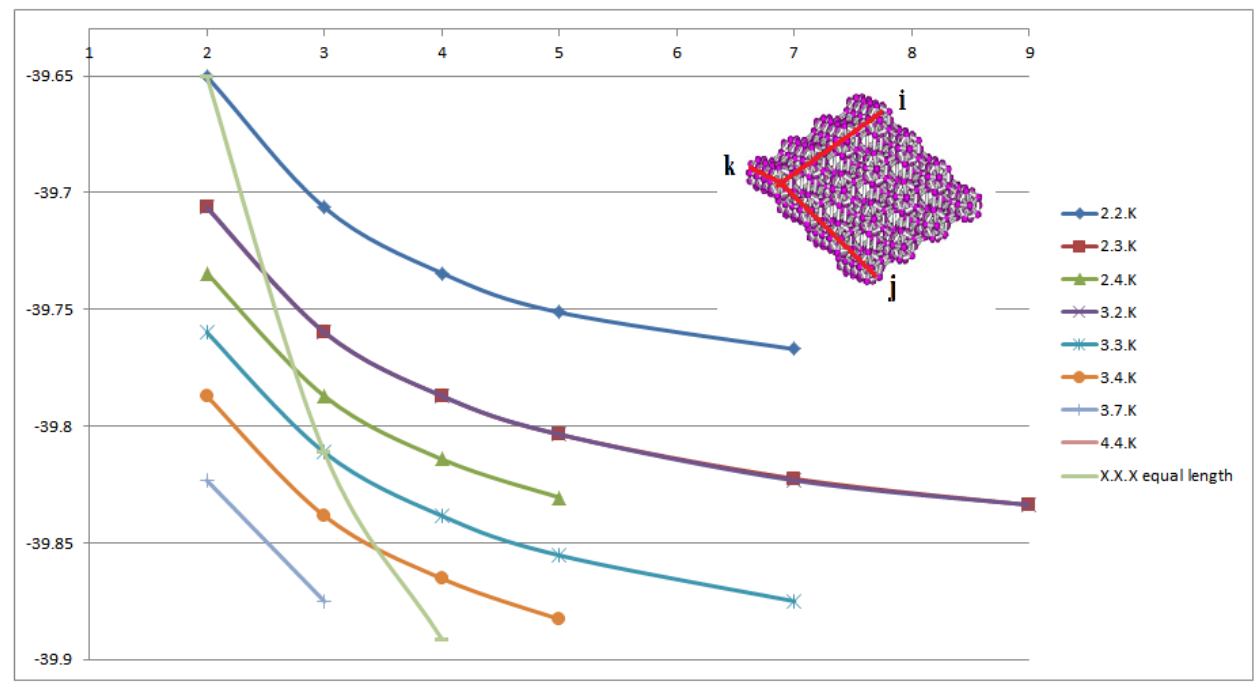

Figure 37. Rhombohedra cuts of alpha boron

\begin{tabular}{|r|r|r|r|r|r|}
\hline structure & gap (eV) & structure & gap (eV) & structure & gap (eV) \\
\hline $2.3 .5:$ & 0.0039 & $3.2 .9:$ & 0.0058 & $4.4 .4:$ & 0.0038 \\
\hline $2.4 .4:$ & 0.0014 & $3.3 .4:$ & 0.0027 & & \\
\hline $2.4 .5:$ & 0.0046 & $3.4 .3:$ & 0.0032 & & \\
\hline & & $3.3 .5:$ & 0.0029 & & \\
\hline & & $3.4 .4:$ & 0.0023 & & \\
\hline & $3.4 .5:$ & 0.0028 & & \\
\hline & $3.7 .2:$ & 0.0017 & & \\
\hline & $3.7 .3:$ & 0.0015 & & \\
\hline
\end{tabular}

Figure 38. HOMO-LUMO gaps for select 3D rhombohedral cuts of alpha boron

One can see from figure 39 that as the structures get larger the energy tends to decrease finally reaching the limiting value of approximately $-40.12 \mathrm{eV}$. The energy converges slowly with size to that value, requiring what appears to be millions of atoms. For example, a 43_43_43 structure has $12^{\star} 43^{*} 43^{*} 43=$ 954,084 a million atoms, about a 23 nanometer structure. One also notes that 
when the aspect ratio is skewed heavily the energy is much higher. For example from the plot, 2_2_7, which is a 7 ball length bar, is about the same energy as 3_3_2 but has 336 atoms and 3_3_2 only has 216 atoms.

This study demonstrates that for rhombohedral cuts of boron, ones that would probably be accessible today in a lab, compact structures, not high aspect ratio structures, tend to be lower in energy. This is expected, and now demonstrated. Combined with the simulated annealing result it is also noted that if you heated a 2_2_7 bar to over thousand degrees it will not reconstruct to a more compact icosahedral symmetry structure easily, it will most likely reconstruct to an amorphous structure instead. This is because the icosahedrals in the structure will collapse instead of float around and migrate.

The implication of this may be that the construction of small boron clusters with rhombohedral low energy structures may be best achieved via mechanical means like ablation, and not high temperature means like deposition.

\subsubsection{D structures of icosahedra with equal aspect ration}

In the previous case it was mentioned that if the aspect ratio is low, the energy of the structure in question is low. Boron favors compact structures as long as the unit is icosahedral. An energy plot of $i \_i$ i is shown in figure 39 and compared to the limiting case of alpha boron. 

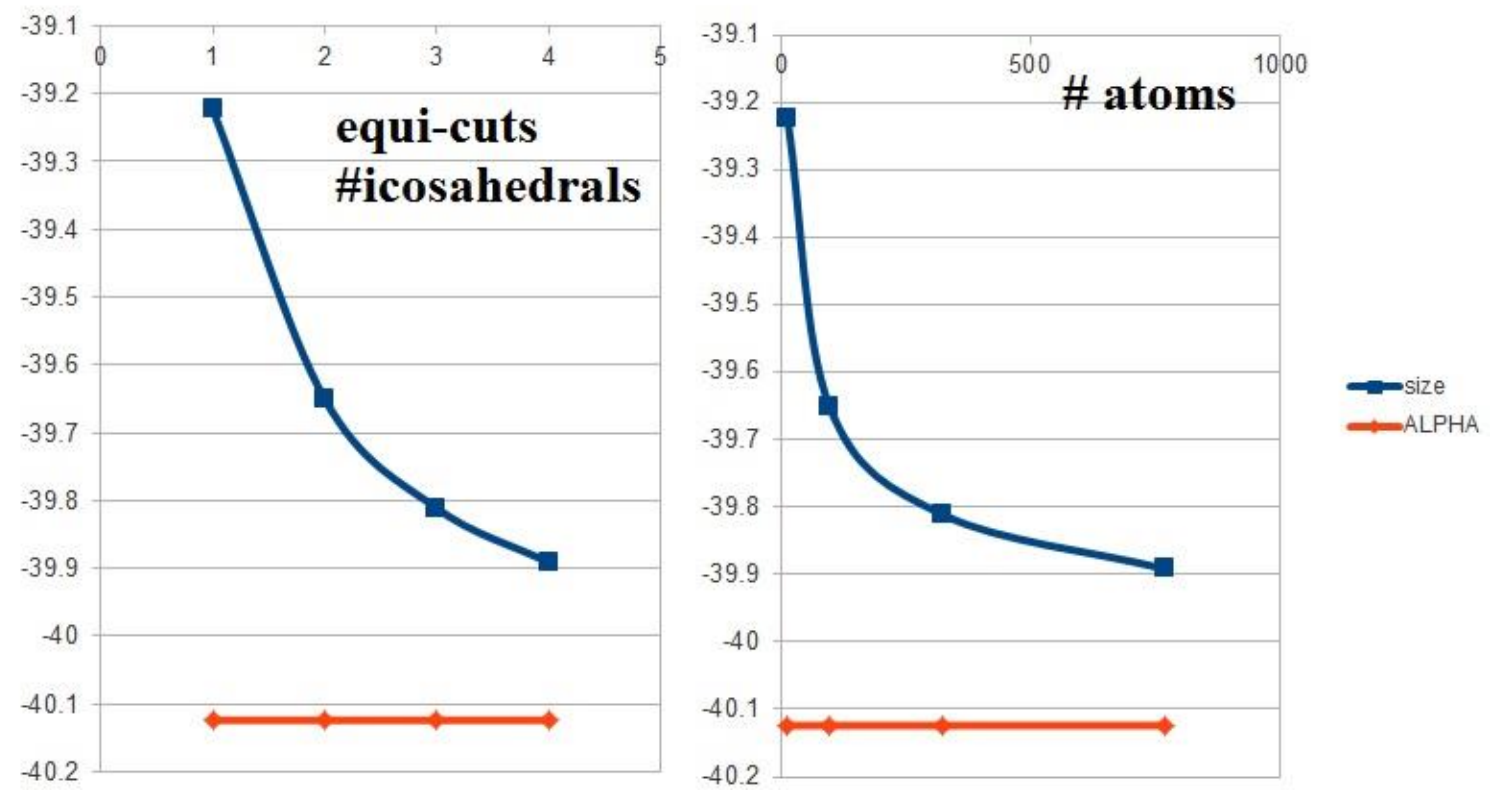

Figure 39. Equi-cuts of boron alpha with rhombohedral symmetry

This curve represents a limiting envelope for rhombohedral cut structures of all types. A rhombohedral cut of complete icosahedrons cannot be lower in energy than this for a given fixed number of atoms. This is not true for spherical cuts or structures that do not retain the rhombohedral symmetry.

\subsection{ALPHA BORON CUT CLUSTERS: SPHERICAL SYMMETRY}

\subsubsection{Spherical cuts of alpha boron}

We next consider spherical cuts of alpha boron. These would be cuts made by picking an interior point in a bulk slab of alpha boron and sweeping out a sphere from that origin. The spherical styled slice would cut through many icosahedra leaving only the icosahedra in the deepest interior intact. Initially this 
would create a scenario with lots of loose atoms on the surface. These loose atoms would reconstruct along the surface and fit into the interstitial locations in the new surface.

During the simulations it was noted that if the cuts were large enough to encompass more than one connected icosahedra, those icosahedra and the connections between them were stable and never reconstructed within the interior of the structure. This was true even when heated significantly.

It was instead observed that if heated beyond a critical temperature, the structure would become fully amorphous. In other words the icosahedra would collapse before they would disconnect from one another. This is also was also true, as noted in the previous section, for rhombohedral cuts. Surface atoms would move dramatically and reconstruct under heat up to $1000 \mathrm{~K}$, but it was never observed that they would combine into icosahedra themselves. Select small and large spherical clusters are shown in figures 41.

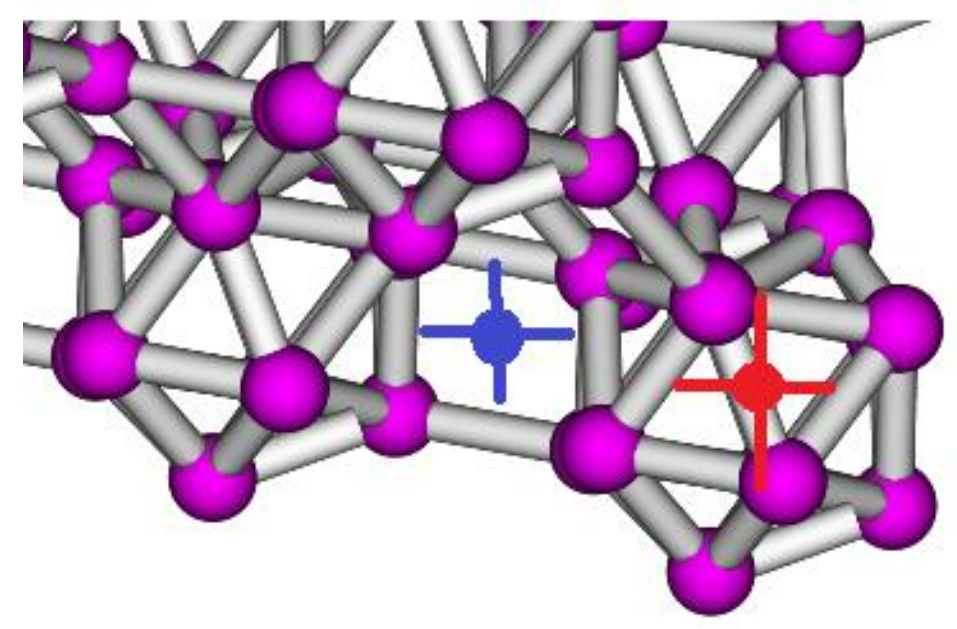

Figure 40. Red is centered on ball, Blue is centered between balls 
There are two cases; one where the origin of the cut is centered on an icosahedral ball, the other case is where the origin of the cut is centered between an icosahedra. See figure 40.

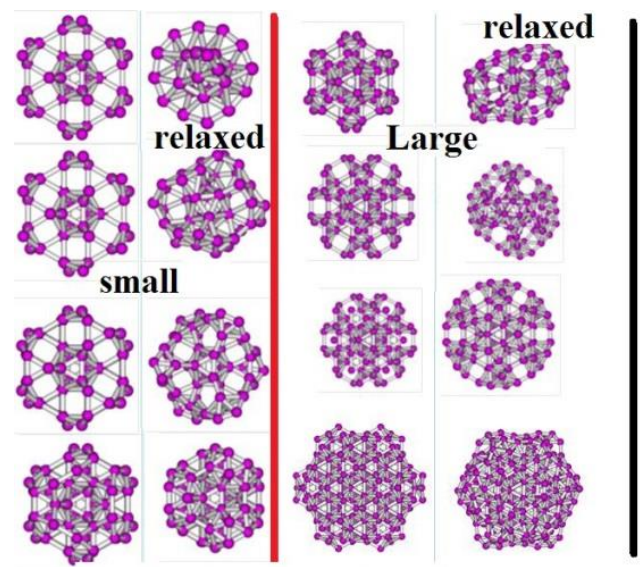

Centered upon an icosahedra

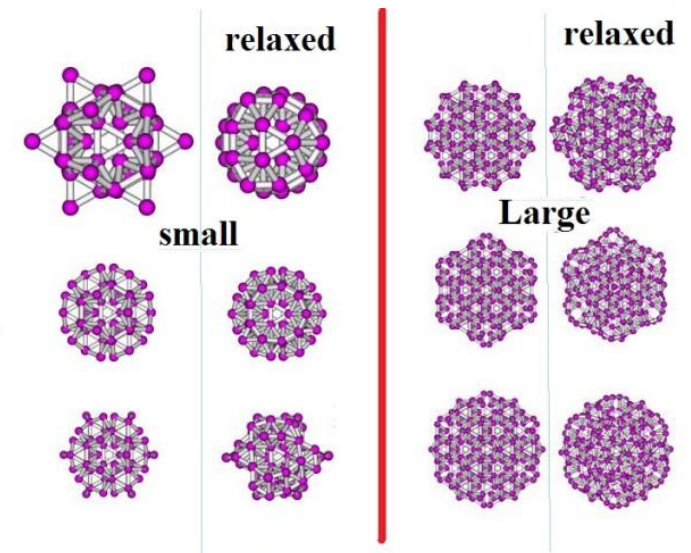

Centered between an icosahedra

Figure 41. Examples of large/small spherical cuts before and after relaxation

Figure 41 shows small and large pre and post relaxation spherical cut boron clusters. Every cluster centered upon an icosahedra has at least one ball in the very center. The largest images have several.

As the radius of the cut gets larger, the number of icosahedra in the interior of the cluster increases. In the limit as the cut get extremely large, the number of surface atoms that are not part of a complete 12 atom icosahedra, become small compared to the number of atoms that belong to a complete icosahedra. This is true also for the case of cuts that are centered between the icosahedra. As the number of atoms shrinks, in the case of the spherical cut centered between icosahedra, there is not one complete 12 atom icosahedra 
present in the cluster. These smaller clusters relax to very interesting shapes and some are quite symmetric while others seem to collapse to an amorphous state.

If we look at the energy, as shown in figure 42 we see a trend. For a fixed number of atoms below 100 we see that the case of the spherical cut centered between icosahedra tends to be lower in energy. At approximately 100 atoms the spherical cut case centered on an icosahedra, starts to be lower in energy. As the number of atoms in either styled cut nears 400 , both cases tend to trend to a similar energy. This makes sense because after we get to approximately 100 atoms, the case centered on icosahedra starts to form a second internal layer of full icosahedra, dropping the energy. When we get to 400 atoms both cases have lots of complete icosahedra so they are competitive in energy. This interesting result implies that very small, but over 100 atoms, boron structures will most likely contain complete icosahedra in the interior as opposed to a fullerene-like structure. 


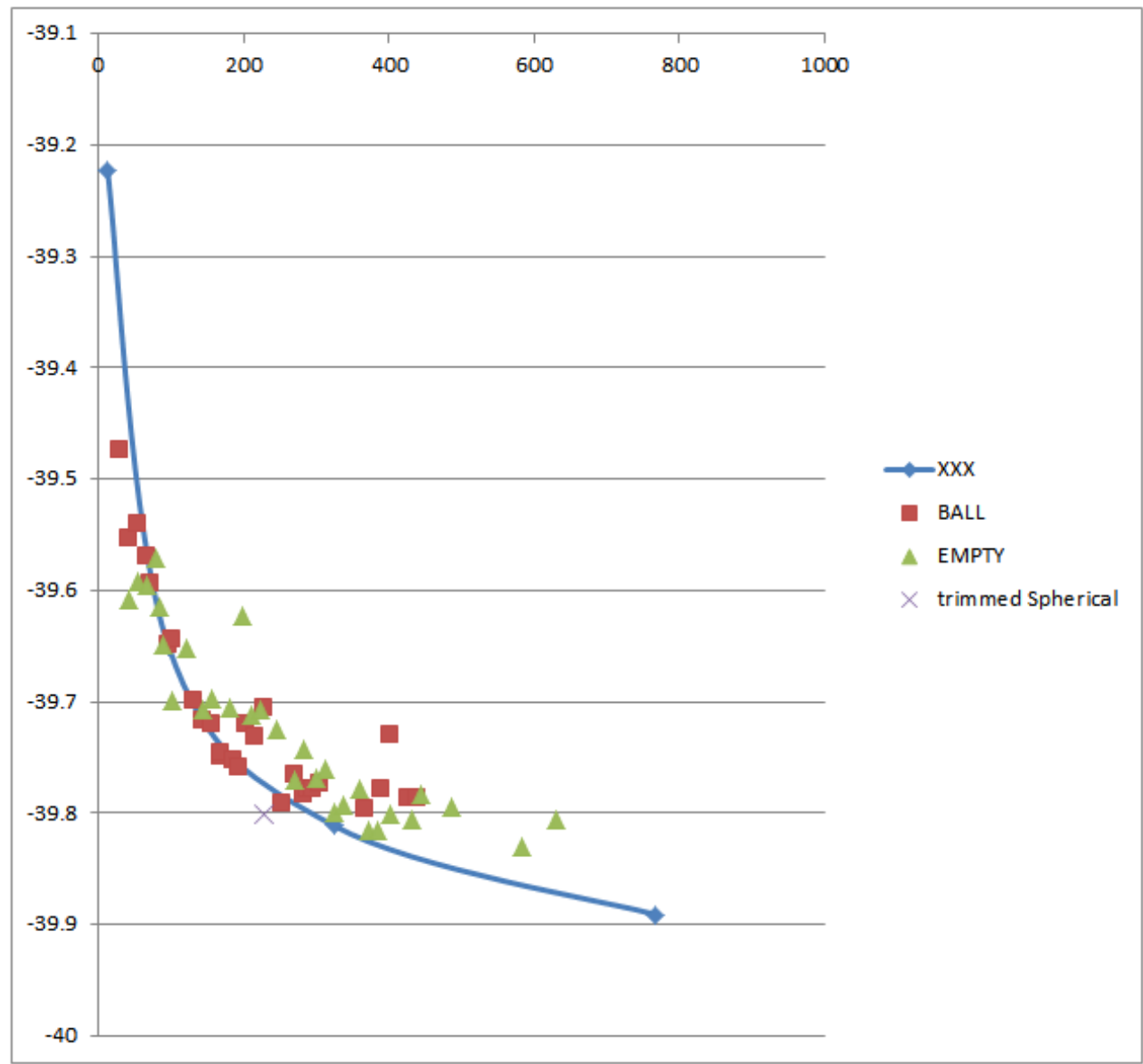

Figure 42. Energy plot of boron alpha spherical plots

A table of the homo-lumo gaps for various spherical cuts of both type reveal that all spherical cuts are metallic, see figure 43. 


\begin{tabular}{|l|r|l|r|}
\hline structure & Gap(eV) & structure & Gap(eV) \\
\hline BALL.6.5: & 0.01098464 & EMPTY.4.3: & 0.10456896 \\
\hline BALL.6.8: & 0.16452458 & EMPTY.4.6: & 0.00012138 \\
\hline BALL.7.1: & 0.00609743 & EMPTY.4.9: & 0.06742697 \\
\hline BALL.7.6: & 0.02249012 & EMPTY.5.3: & 0.07476345 \\
\hline BALL.8.2: & 0.02698676 & EMPTY.6.1: & 0.12669239 \\
\hline BALL.8.3: & 0.0229769 & EMPTY.8.1: & 0.03689687 \\
\hline BALL.8.6: & 0.0421953 & EMPTY.8.4: & 0.01056585 \\
\hline BALL.8.7: & 0.11099629 & EMPTY.8.5: & 0.02235947 \\
\hline BALL.8.9: & 0.18862395 & EMPTY.8.6: & 0.00897602 \\
\hline BALL.9.0: & 0.01418726 & EMPTY.8.7: & 0.02553286 \\
\hline BALL.9.1: & 0.02153193 & EMPTY.8.9: & 0.02914311 \\
\hline BALL.9.2: & 0.01802314 & EMPTY.9.0: & 0.01044613 \\
\hline BALL.9.3: & 0.03014062 & EMPTY.9.1: & 0.03699231 \\
\hline BALL.9.4: & 0.00946142 & EMPTY.9.2: & 0.00890715 \\
\hline BALL.9.7: & 0.01269267 & EMPTY.9.4: & 0.03010392 \\
\hline BALL.9.9: & 0.00486933 & EMPTY.9.5: & 0.08641846 \\
\hline BALL.10.0: & 0.00388649 & EMPTY.9.6: & 0.01665902 \\
\hline BALL.10.2: & 0.01873569 & EMPTY.9.7: & 0.01142165 \\
\hline BALL.10.3: & 0.01974498 & EMPTY.9.9: & 0.01076816 \\
\hline BALL.10.6: & 0.01483926 & EMPTY.10.1: & 0.00895999 \\
\hline BALL.10.7: & 0.00871211 & EMPTY.10.2: & 0.00598843 \\
\hline BALL.10.8: & 0.01548558 & EMPTY.10.4: & 0.00574742 \\
\hline & EMPTY.10.5: & 0.00564568 \\
\hline & EMPTY.10.7: & 0.00725355 \\
\hline
\end{tabular}

Figure 43. Plot of all spherical cuts homo-lumo gaps

\subsubsection{Trimmed spherical cuts of alpha boron}

In the previous section loose surface atoms left over from the spherical cut which were not part a complete 12 atom icosahedra, were allowed to move around and reconstruct. If instead those atoms are removed from the cut cluster, 
we would have a compact spherical shape of icosahedra with no partial icosahedral structures. This case was run for the example of 228 atoms or 19 icosahedra see figure 44 . In figure 44 , the figure on the left is the initial structure and the figure on the right is relaxed structure-they are extremely similar. It is noted that this is a spherical cluster, not a rhombohedral structure, and is more compact. It does not look spherical because it is too small, but it is the most compact alpha cut tested.
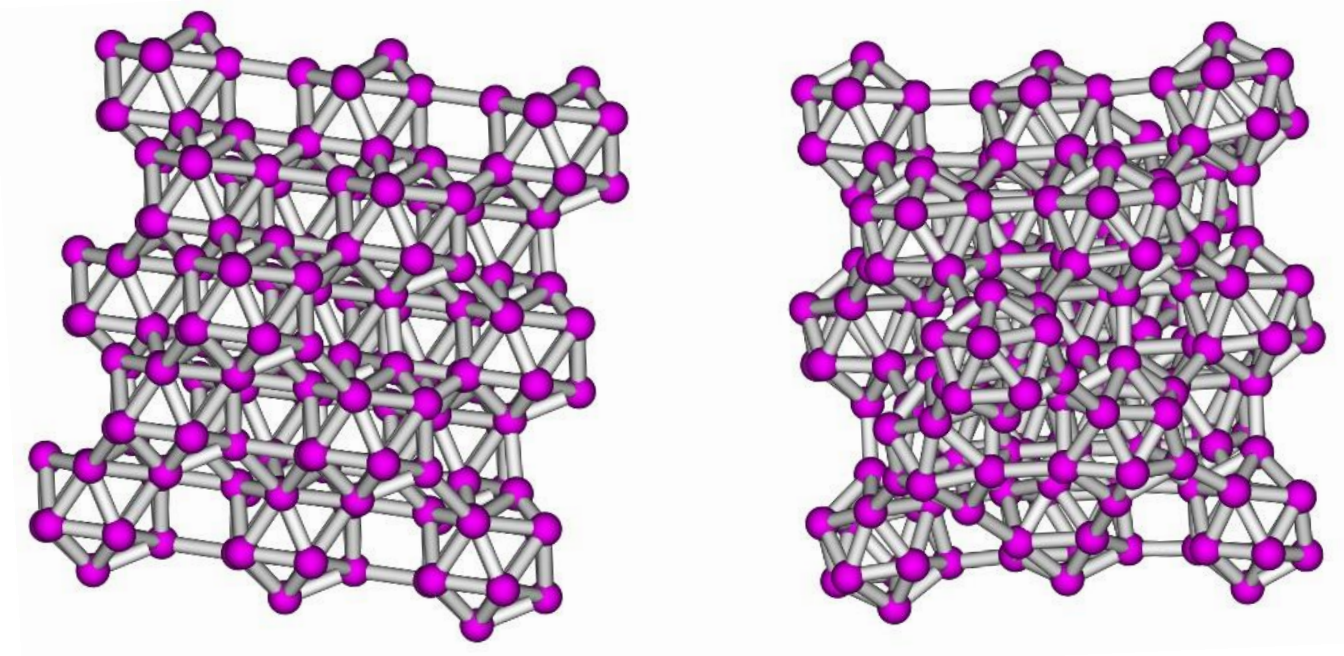

Figure 44. Trimmed spherical cut of alpha boron

The outer corners do reconstruct slightly, but the general shape stays the same. This is the lowest energy cluster I have ever found for an alpha boron cut. It is compact, fully made of icosahedra, spherical and resistant to reconstruction up to about $1000 \mathrm{~K}$. Below in figure 45 we compare all structures including the boron 80 buckyball on one chart. 


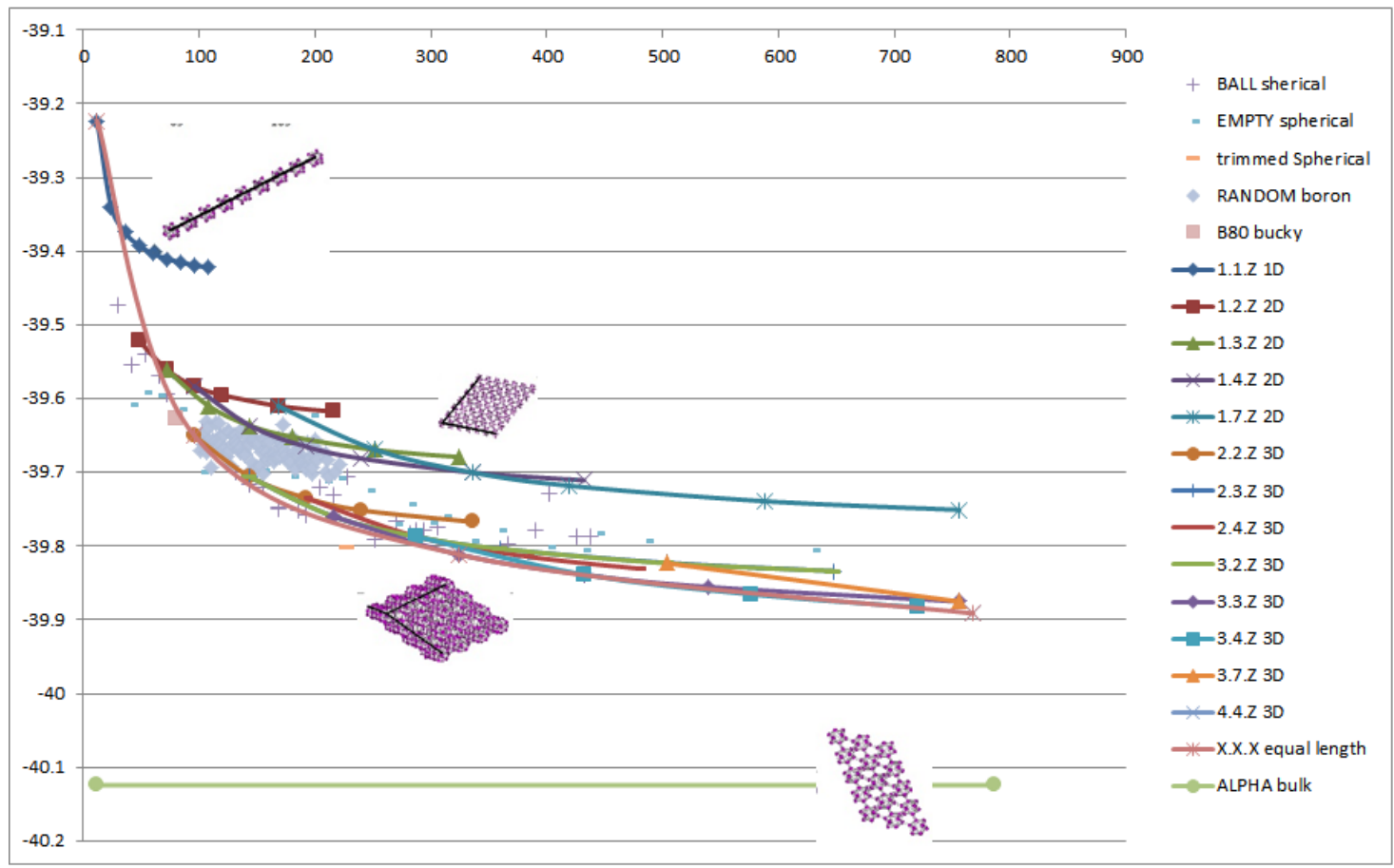

Figure 45. All alpha boron cuts, random clusters, bulk boron alpha, and the $B 80$ buckyball.

The B80 buckyball (B80 bucky) is competitive in energy with random structures (RANDOM boron). The equi-cut limiting envelope (X.X.X equal length) contains most clusters, with a few exceptions such as several small spherical cut boron clusters (BALL spherical) and the trimmed spherical cuts (trimmed spherical) mentioned above. These clusters must have some atoms on the surface that create a lowering of the energy scenario and are worth further exploration. Next we will analyze select clusters that are about 220 atoms in size. 


\subsection{LOCAL ANALYSIS OF BORON CLUSTERS OF SIMILAR SIZE}

It is informative to compare several different structures of similar size. 5 different types of clusters have been selected, all have around $\sim 220$ atoms:

1. 252 atom spherical cut centered on an icosahedra (BALL)

2. 216 atom spherical cut centered on an icosahedra (BALL)

3. 228 atom trimmed spherical cut (trimmed)

4. 228 atom random amorphous cluster (RANDOM)

5. 210 atom spherical centered between icosahedra (EMPTY)

A plot of the energy of each cluster is shown in figure 46 . All are metallic, the homo-lumo gaps are less than $0.01 \mathrm{eV}$. Because of this a DOS states plot may not tell us much, but by looking at the pair/angle distribution function we will get some insight into the type of bonding we have.

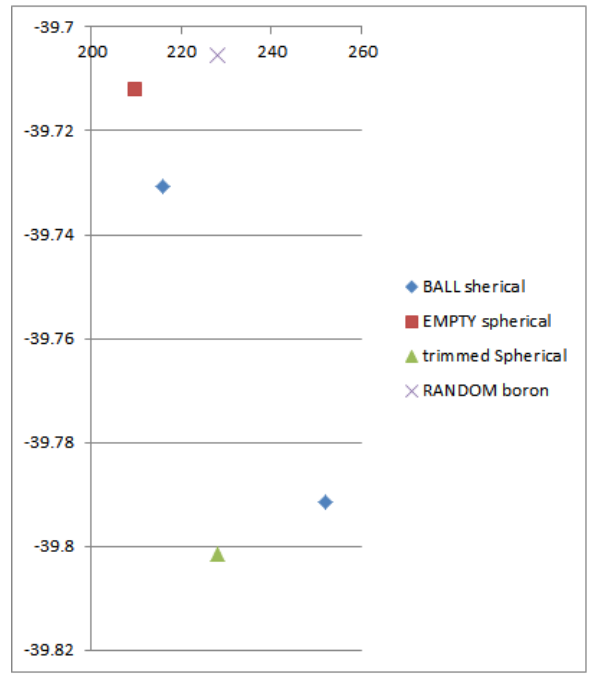

Figure 46. Energy/atom of select clusters of size 220 atoms 
The pair distribution function is shown in figure 47.
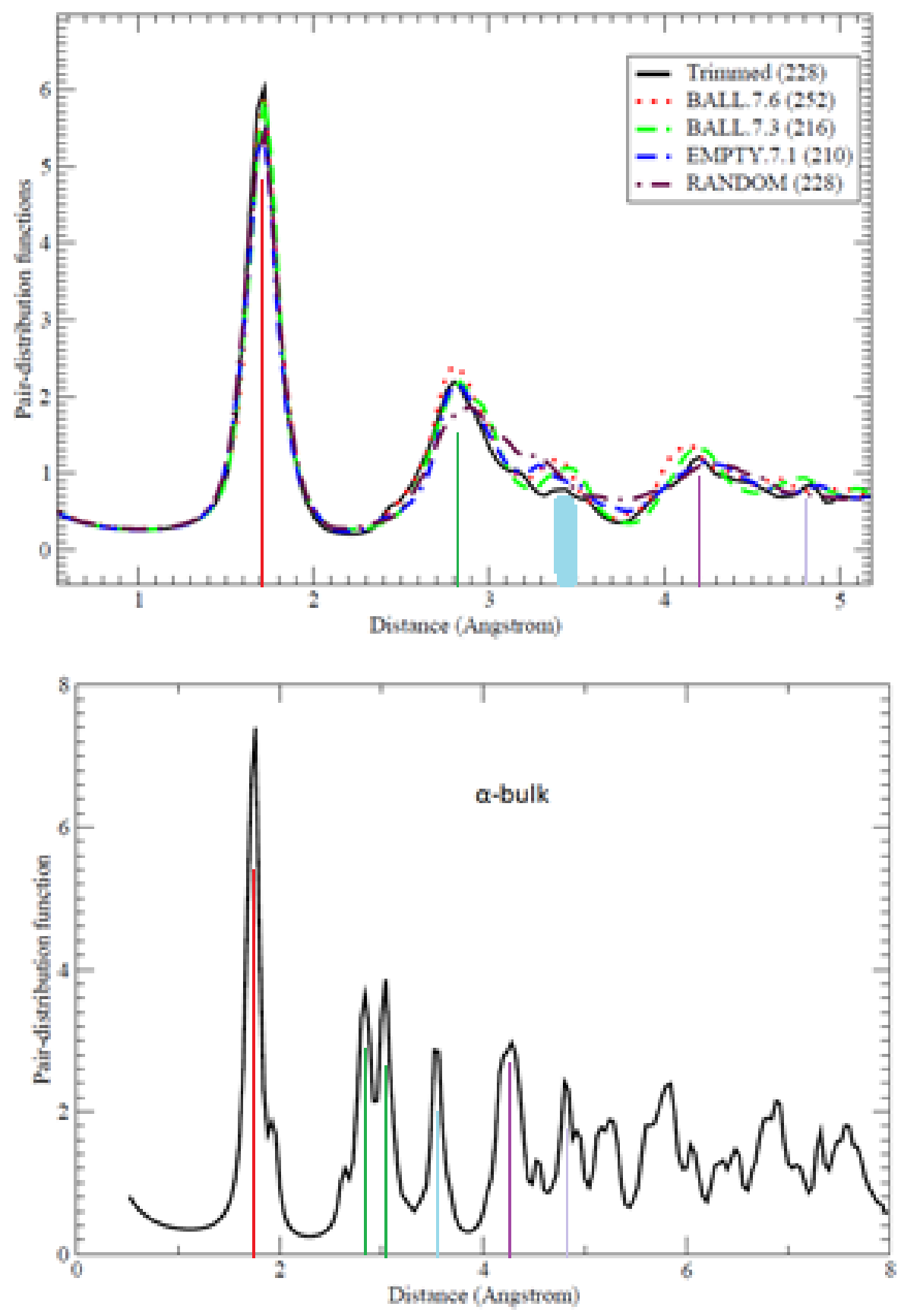

Figure 47. Pair Distribution function for the 5 test clusters.

It is immediately apparent that the trimmed spherical cluster (lowest in energy/atom) has the highest sharpest peaks, the random cluster (highest in 
energy/atom) has the broadest lowest peaks. Alpha boron has very distinct sharp peaks that are obviously periodic (red). The $3^{\text {rd }}$ peak (wide light blue) in the cluster set varies the most, this is also the sharpest peak of the first 5 peaks. The double peaks (green) in alpha boron are present as a broadened single peak in all of the clusters. The double-peak is the tell-tale signature of crystalline alpha boron; this double peak is not present in clusters, instead we see a broadened single peak in all of the clusters examined. The closest cluster to mimic alpha boron is the trimmed case. This makes physical sense, the first sharp peak designates the boron bond length of the icosahedra in boron alpha: $1.7556,1.6875,1.7358$, and 1.691 angstroms. This length is also prevalent in the 5 test clusters, but to a lesser degree due to reconstructions. It is most prevalent in the trimmed cluster because it is solely made of icosahedra, and least prevalent in the random structure due to the random smearing effect in that cluster.

Next we look at the angular distribution function. First for the 5 clusters, see figure 48. 

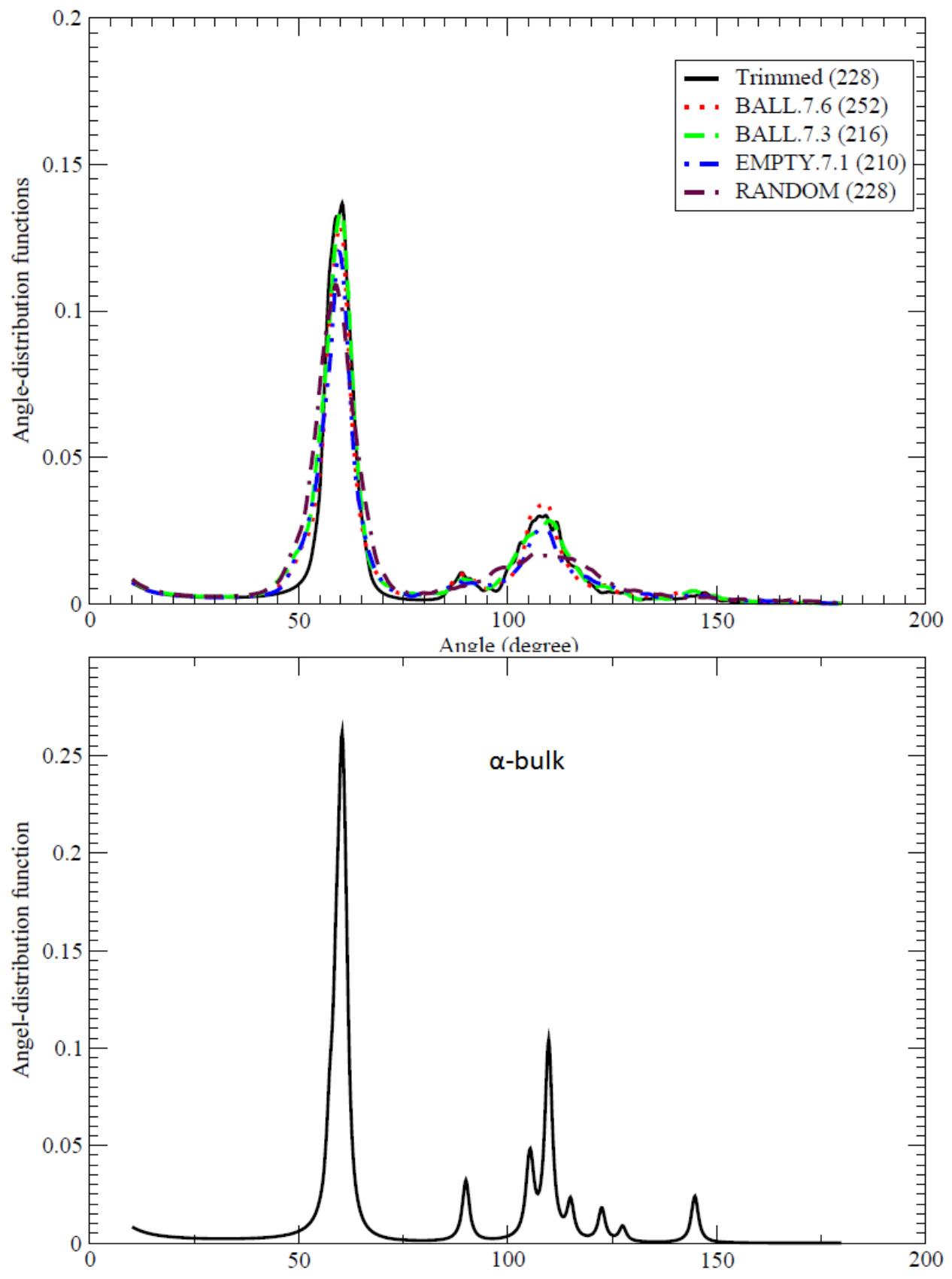

Figure 48.Angular distribution function for the 5 test clusters.

A similar situation is noted in the angular distribution function. Boron alpha is comprised of many 60 degree angles due to the numerous equilateral triangles in the icosahedra. Secondly, the triangles do not lay flat they are wrapped over a 
ball, the angle they make as they bend is 106 degrees, the angle that is made between the icosahedra and connection line to the next icosahedra is about 115 degrees. This accounts for the 3 major angles in boron alpha. We also see the same 60 degree bonding heavily in all of the boron clusters. This makes perfect sense, unlike silicon that is loaded with lots of tetrahedral bonding, we see the true nature of boron with heavy three center triangular bonding.

This concludes chapter 3 and the discussion of purely homogenous boron structures. In conclusion it is noted that the lowest energy cluster observed was that of a compact spherically shaped boron cluster comprised solely of 12 atom icosahedra. All pure boron clusters exhibit similar bonding as evidenced by the local analysis. 


\section{CHAPTER IV: PHOSPHORUS PARAMETER}

\section{ACQUISITION}

\subsection{PARAMETER FITTING OF PHOSPHORUS}

\subsubsection{Cluster set used in the fitting of phosphorus}

Following in the same path as the boron work we will find a crude but very effective set of parameters for phosphorus. Improvement on this set is always possible given more time, but the point was to use phosphorus as a dopant in silicon. Given that, we will not spend a lot of time analyzing phosphorus since the bonding scenarios and large phosphorus structures are not as complicated. Likewise this section will not discuss in detail the fitting process because it mirrors the boron fitting process described in prior sections with fewer rigors.

The fitting cluster set is shown in figure 49 , and was not changed. The parameter fit to the phosphorus clusters was quite good and fairly easy to achieve especially compared to boron. There are many detailed articles about small phosphorus clusters ${ }^{86-90}$ that can be utilized as an initial guide. Every cluster was first calculated using DFT with the hybrid B3LYP functional and the aug-cc-pvTZ basis set. After geometry optimization, the nuclear cores were fixed and coupled cluster calculations were performed using the $\operatorname{CCSD}(T)$ method with 
the cc-pvDZ and cc-pvTZ when possible. This is a very computationally intensive calculation, thus extremely large basis sets cannot be used.
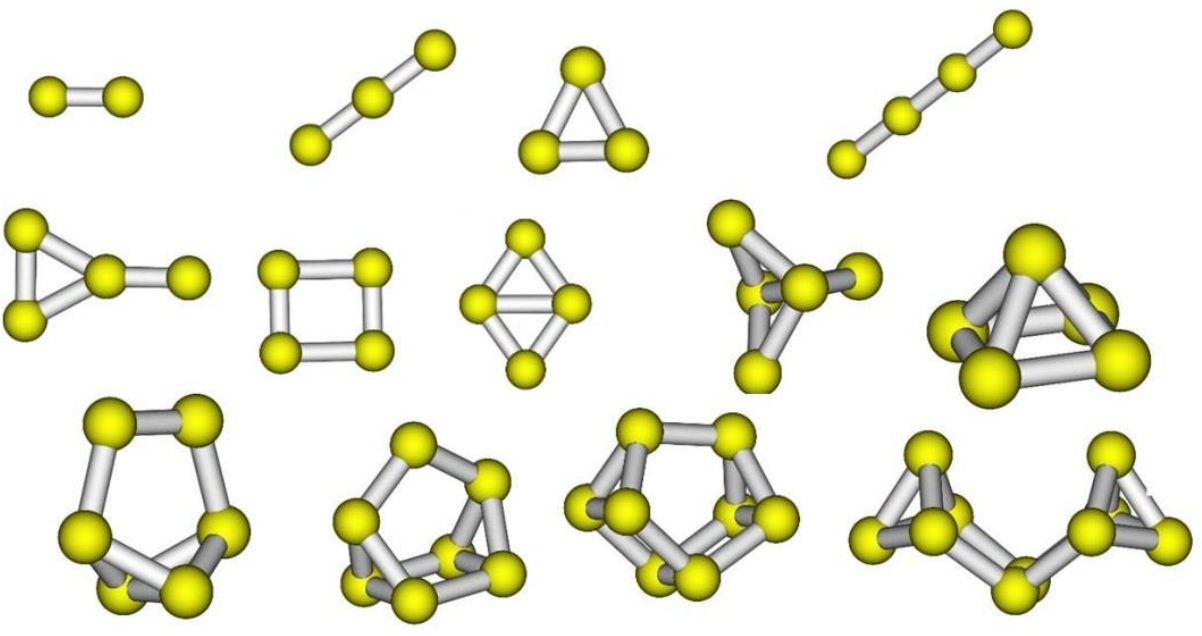

Figure 49. Clusters used in the phosphorus fitting process

Next we needed an extended phase to expand the fitting database. The easiest phase found in nature at room temperature is the black phosphorus phase. In the next section a fit to black phosphorus will be performed.

\subsubsection{Black phosphorus: An extended phosphorus phase}

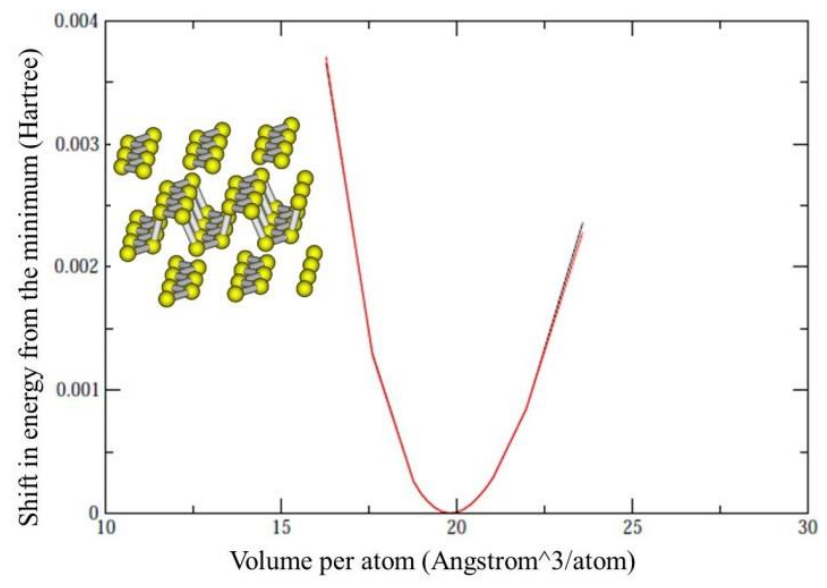

Figure 50. The black phosphorus phase 
The black phosphorus phase is a common phase in found in nature ${ }^{91}$. It is very well understood ${ }^{92}$. The parameter fit for this phase is exceedingly strong as evidenced in figure 50 . The curve shown is actually two curves that lay so closely on each other that it appears to be one curve. This is more than sufficient for our phosphorus Hamiltonian. This phase was calculated using DFT (VASP ${ }^{32}$ ) using GGA approach.

\subsubsection{Optimized phosphorus overlaps}

The final optimized phosphorus overlap curves are typically shaped, as shown in figure 51 , with reasonable bond length cutoff.

\section{PHOSPHORUS OVERLAPS}

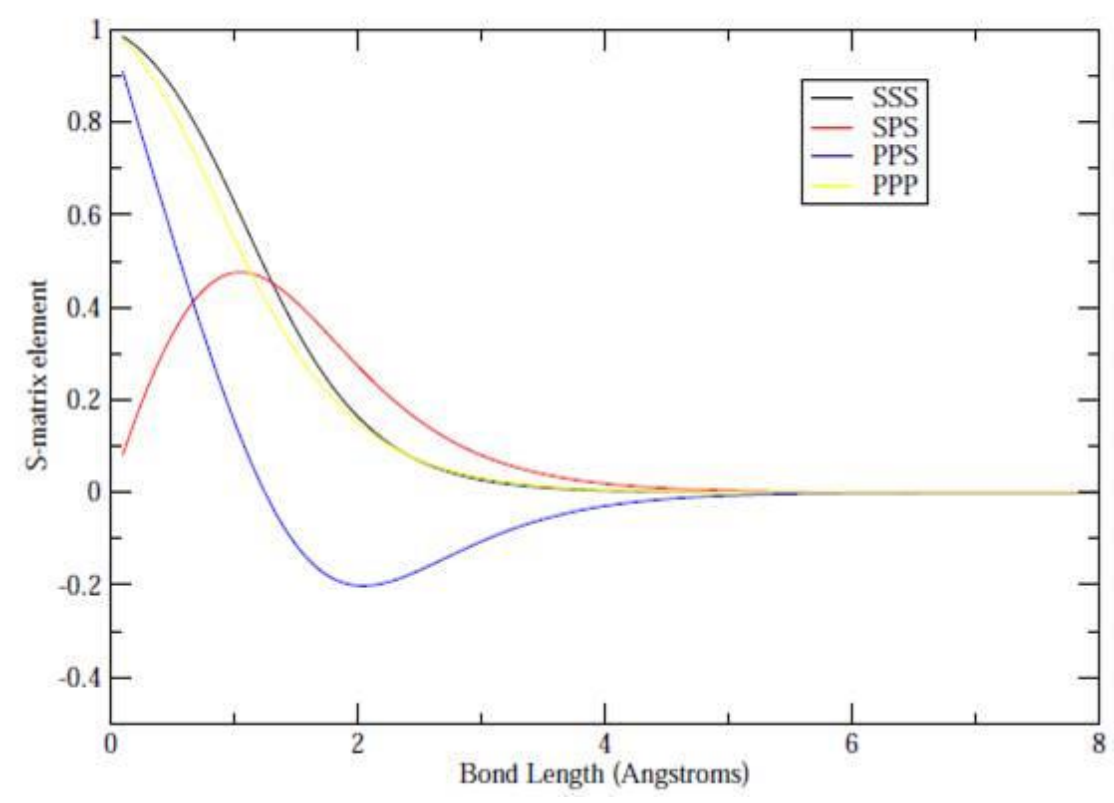

Figure 51. Overlap curves for phosphorus 


\subsubsection{Predictions}

A secondary database ${ }^{71}$ for the robust check was created consisting of intermediate sized clusters of phosphorus 10, 11, 12, 13, 14, 15 and 24 atoms ${ }^{88,93}$, see figure 52 . These clusters were used as a robust check. Once a parameter set was found, see figure 49 , which met all requirements and passed this robust check it was deemed ready to use. Its main application would be a single dopant atom in a silicon wire, but, this phosphorus Hamiltonian could easily be applied to phosphorus structures of arbitrary size.

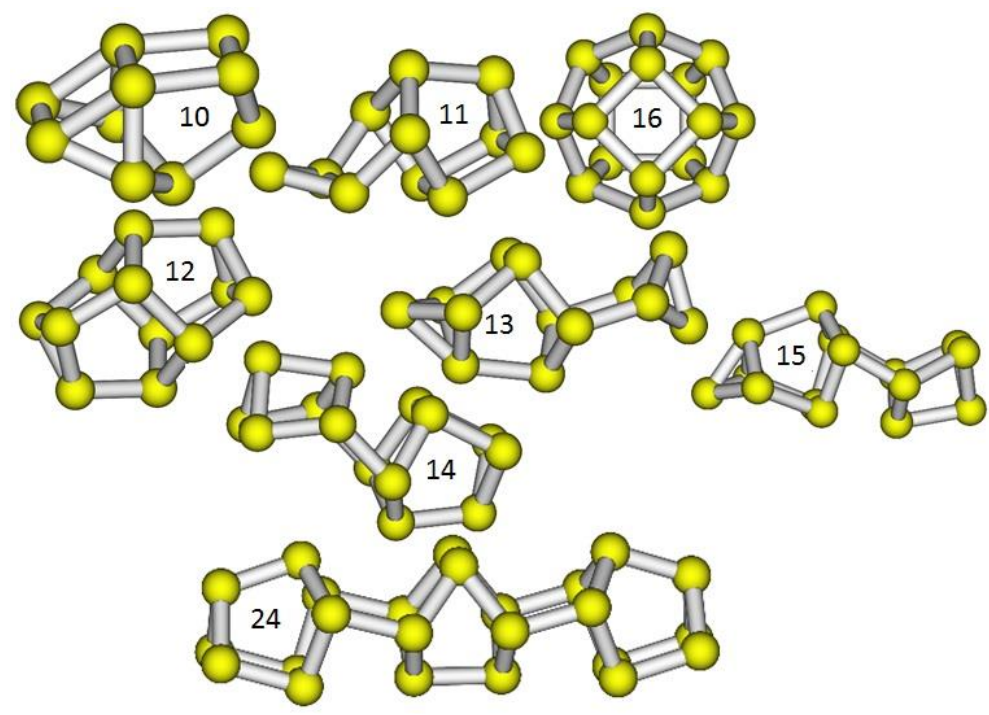

Figure 52. Predicted clusters from the phosphorus parameter set

\subsubsection{Comparison with DFT}

The robust check yielded reasonable agreement with DFT as shown in figure 49. The shapes of the clusters are very consistent with DFT as shown in figure 53 . 


\begin{tabular}{|c|c|c|}
\hline $\begin{array}{c}\text { \# of } \\
\text { atoms }\end{array}$ & $\begin{array}{c}\text { DFT } \\
\text { BE(eV) }\end{array}$ & $\begin{array}{c}\text { SCED } \\
\text { BE(eV) }\end{array}$ \\
\hline 10 & -4.589 & -4.364 \\
11 & -4.581 & -4.312 \\
12 & -4.616 & -4.457 \\
13 & -4.529 & -4.342 \\
14 & -4.613 & -4.382 \\
15 & -4.557 & -4.354 \\
16 & -4.549 & -4.479 \\
24 & -4.645 & -4.432 \\
\hline
\end{tabular}

Figure 53. Comparison of binding energy with DFT and final

parameter set

\subsubsection{Conclusions about the final parameters set}

The phosphorus parameter set was found very rapidly, would work well for intermediate sized clusters, and it will be shown that it works well for the case of a phosphorus dopant atom in silicon. Optimized phosphorus parameters are shown in figure 54 along with optimized boron parameters.

\begin{tabular}{|c|c|c|c|c|c|c|c|}
\hline $\begin{array}{l}\text { Hamiltonian } \\
\text { Parameters } \\
\end{array}$ & PHOSPHORUS & $\begin{array}{l}\text { Hamiltonian } \\
\text { Parameters } \\
\end{array}$ & PHOSPHORUS & $\begin{array}{c}\text { Overlap } \\
\text { Parameters } \\
\end{array}$ & PHOSPHORUS & $\begin{array}{c}\text { Overlap } \\
\text { Parameters } \\
\end{array}$ & PHOSPHORUS \\
\hline$\varepsilon_{s}$ & -19.22000000 & $U$ & 10.47241785 & $\alpha_{p p \pi}$ & 1.78785042 & $B_{s p \sigma}$ & 0.81363400 \\
\hline$\varepsilon_{p}$ & -9.54000000 & $\alpha_{K}$ & 0.14765164 & $\alpha_{p p \sigma}$ & 1.89060543 & $B_{s s \sigma}$ & 0.05272676 \\
\hline$\varepsilon_{s}^{\prime}$ & -16.66589734 & $A_{N}$ & -0.73310684 & $\alpha_{s p \sigma}$ & 1.62037618 & $d_{p p \pi}$ & 0.49274243 \\
\hline$\varepsilon_{p}^{\prime}$ & -13.65435941 & $B_{N}$ & -13.91643958 & $\alpha_{s s \sigma}$ & 1.84042503 & $d_{p p \sigma}$ & 1.65351279 \\
\hline$W_{p}^{0}$ & -0.30130993 & $\alpha_{N}$ & 2.76104487 & $B_{p p \pi}$ & 0.38771518 & $d_{s p \sigma}$ & 0.87591292 \\
\hline$W_{s}^{0}$ & 0.31052577 & $d_{N}$ & 0.13003016 & $B_{p p \sigma}$ & -0.75723800 & $d_{s s \sigma}$ & 0.99996154 \\
\hline$\alpha_{p, w}$ & 0.77793196 & $B_{Z}$ & 1.67738974 & & & & \\
\hline$\alpha_{s, w}$ & 0.72083974 & & & & & & \\
\hline
\end{tabular}

Figure 54. Optimized Phosphorus parameters shown in comparison to boron parameters 
Due to minimal testing, the phosphorus Hamiltonian may have difficulty in some scenarios where energy differences are very small. These parameters have not been verified and validated at the level that the boron parameter set. If a large phosphorus cluster study was to be performed, an expanded robust check would need to be employed, to capture small energy differences.

In the next chapter the boron and phosphorus Hamiltonians will be used in conjunction with a previously developed silicon Hamiltonian ${ }^{8,94,95}$. In this application boron and phosphorus will be used as dopant atoms in a silicon multitwin nanowire. 


\section{CHAPTER V: DOPING THE SILICON WIRE WITH BORON AND PHOSPHORUS}

\subsection{THE HETEROGENEOUS HAMILTONIAN AND OVERLAP}

Until this point, all systems considered have been either comprised solely of boron or phosphorus. In chapter one, an assumption was made that all atoms in the system would be of the same elemental type. For the heterogeneous case where there is more than one type of element we must modify our Hamiltonian and overlap to reflect this.

The goal of the SCED-LCAO methodology is to create an extremely flexible and reliable Hamiltonian and overlap, yet not increase the number of parameters in the system beyond what is absolutely necessary. Although one could create a heterogeneous database of pair interaction based parameters, such as boron/silicon parameters, this defeats the purpose of the SCED-LCAO. That approach will quickly escalate the problem to an intractable level unnecessarily. Our desire is to construct a SCED-LCAO Hamiltonian for binary systems that is based upon the SCED-LCAO formulation for homogeneous (single element) systems, see chapter 1. The Hamiltonian/overlap will be 
comprised of functions that will mix homogeneous parameters in a prescribed manner.

The first attempt made was by Dr. Yu Ming using a 50/50 mixing ratio with the assumption that carbon chemistry and silicon chemistry are somewhat similar based upon the location in the periodic table. I also tried a 50/50 with boron and silicon. It was soon realized that the model could be improved by noting that indeed charge transfer effects will play a significant role - and this may not be represented well for a 50/50 mixing scheme. If the electronegativity of the two elements is not equivalent, charge will have a preference to move from one atom to another. This has the effect of shifting the energy of a given atomic site, and thus the force. This phenomenon should be included in the Hamiltonian and is the motivation for introducing the mixing parameter $\delta_{i j}$.

Parameters $\varepsilon_{i \alpha}, \varepsilon_{i \alpha}^{\prime}$ and $U_{i}$ are site specific. The function $W_{i \alpha}\left(R_{i j}\right)$ is also site specific. All other phenomenological functions are dependent upon binary pairs of sites. The way these binary site dependent functions are treated is the subject of current research in the CMT group.

For this body of work, weighted averaging of homogenous parameters or functions has been chosen. The weighting factor, or mixing parameter $\delta_{i j}$, is the same value in every function. 


\begin{tabular}{|r|rrrr|rr|}
\hline \multicolumn{1}{|c}{ electronegativity } & \multicolumn{2}{c}{2.04} & 2.19 & 1.90 & 2.55 & 2.20 \\
\cline { 2 - 8 } 2.04 & ELEMENT & boron & phosphorus & \multicolumn{1}{c}{ silicon } & \multicolumn{1}{c}{ carbon } & hydrogen \\
\cline { 3 - 8 } 2.19 & boron & 0.00 & 0.15 & -0.14 & 0.51 & 0.16 \\
1.90 & phosphorus & -0.15 & 0.00 & -0.29 & 0.36 & 0.01 \\
2.55 & silicon & 0.14 & 0.29 & 0.00 & 0.65 & 0.30 \\
\cline { 3 - 8 } 2.20 & carbon & -0.51 & -0.36 & -0.65 & 0.00 & -0.35 \\
\cline { 3 - 8 } & hydrogen & -0.16 & -0.01 & -0.30 & 0.35 & 0.00 \\
\hline
\end{tabular}

Figure 55. Electronegativity and differences between elements.

The fact that we will use only one $\delta_{i j}$ per pair of elements in the system is a key feature that reflects not only the need to minimize the number of free fitting parameters, but also the observation that charge transfer and a preference for charge buildup influences every interaction term. The electronegativity of the elements are shown in figure 55. From the chart above we suspect that $\delta_{B P}<0.5$ and $\delta_{B S i}, \delta_{P S i}>0.5$ as a quality, but we do not rigidly make any assumption about the quality or quantity. Environmental effects can greatly influence this parameter beyond the very simple Pauli electronegativity. The system will be optimized to find the best fit $\delta_{i j}$.

$\delta_{i j}$ is found by varying its value from 0 to 1 in conjunction with fixed homogenous parameters. The result is compared with density functional theory results to verify and validate the optimal value for $\delta_{i j}$ for a given binary pair. In our case the pairs are boron-phosphorus, boron-silicon, and phosphorus-silicon. For each pair there must exist a different $\delta_{i j}$. In cases of low charge transfer, the value of $\delta_{i j}$ may be approximated with simply $\delta_{i j}=1 / 2$, and indeed that is a good initial guess in a numerical scheme to find the optimal value of $\delta_{i j}$. If the $\delta_{i j}$ value 
floats too far either towards 0 or 1 , for example $\delta_{i j}=0.1$ or $\delta_{i j}=0.9$, it is highly suspect that this value is not physically realistic.

\subsubsection{Parameter based weighted averaging}

The Hückel type function has an exponential parameter:

$$
K\left(R_{i j}\right)=e^{\alpha_{i, K} R_{i j}}
$$

This parameter is replaced by a weighted average of the homogeneous parameter values.

$$
\alpha_{i j, K}=\delta_{i j} \alpha_{i, K}+\left(1-\delta_{i j}\right) \alpha_{j, K}
$$

Note that if the two atoms are the same $\delta_{i j}=0$ and the expression is reduced back to the homogeneous case discussed in chapter 1.

\subsubsection{Function based weighted averaging}

The function $V_{N}\left(R_{i j}\right)$ and $V_{Z}\left(R_{i j}\right)$ are simple weighted averages of the respective functions.

$$
\begin{aligned}
& V_{N}\left(R_{i j}\right)=\delta_{i j} V_{i, N}\left(R_{i j}\right)+\left(1-\delta_{i j}\right) V_{j, N}\left(R_{i j}\right) \\
& V_{Z}\left(R_{i j}\right)=\delta_{i j} V_{i, Z}\left(R_{i j}\right)+\left(1-\delta_{i j}\right) V_{j, Z}\left(R_{i j}\right)
\end{aligned}
$$

\subsubsection{Parameter based onsite energy weighted averaging}

The overlap functions are parameter averaged, but weighted not only with the parameter $\delta_{i j}$, but also with the onsite energy. 


$$
S_{i j, \tau}\left(R_{i j}\right)=\left(A_{i j, \tau}+B_{i j, \tau} R_{i j}\right) \frac{1+e^{-\alpha_{i j, \tau} d_{i j, \tau}}}{1+e^{-\alpha_{i, \tau}\left(d_{i j, \tau}-R_{i j}\right)}}
$$

In the homogeneous case the set of overlaps is $\tau=s s \sigma, s p \sigma, p p \sigma, p p \pi$ but in general $s p \sigma \neq p s \sigma$ for the general case, thus there are 5 overlap functions instead of $4 \tau=s s \sigma, s p \sigma, p s \sigma, p p \sigma, p p \pi$. The difference in electronegativity of two atoms in the neighborhood of each other will drive charge transfer from the atom with the higher electronegativity to the atom with the lower electronegativity. For example boron and phosphorus should send charge to silicon, and phosphorus would send charge to boron. An onsite energy weighted mean formulation is utilized because we want to fully capture the fact that the bonding state charge will be transferred from the atom with the higher onsite energy to the atom with the lower onsite energy. There is a preference for flow direction - simple averaging will not account for this. This is critical in the binary system because we expect the onsite energies to not be same, or even necessarily similar.

$$
\begin{aligned}
& A_{i j, \tau}=\frac{\delta_{i j}\left|\varepsilon_{i, \mu}\right| A_{i, \tau}+\left(1-\delta_{i j}\right)\left|\varepsilon_{j, v}\right| A_{j, \tau}}{\delta_{i j}\left|\varepsilon_{i, \mu}\right|+\left(1-\delta_{i j}\right)\left|\varepsilon_{j, v}\right|} \\
& B_{i j, \tau}=\frac{\delta_{i j}\left|\varepsilon_{i, \mu}\right| B_{i, \tau}+\left(1-\delta_{i j}\right)\left|\varepsilon_{j, v}\right| B_{j, \tau}}{\delta_{i j}\left|\varepsilon_{i, \mu}\right|+\left(1-\delta_{i j}\right)\left|\varepsilon_{j, v}\right|} \\
& \alpha_{i j, \tau}=\frac{\delta_{i j}\left|\varepsilon_{i, \mu}\right| \alpha_{i, \tau}+\left(1-\delta_{i j}\right)\left|\varepsilon_{j, v}\right| \alpha_{j, \tau}}{\delta_{i j}\left|\varepsilon_{i, \mu}\right|+\left(1-\delta_{i j}\right)\left|\varepsilon_{j, v}\right|} \\
& d_{i j, \tau}=\frac{\delta_{i j}\left|\varepsilon_{i, \mu}\right| d_{i, \tau}+\left(1-\delta_{i j}\right)\left|\varepsilon_{j, v}\right| d_{j, \tau}}{\delta_{i j}\left|\varepsilon_{i, \mu}\right|+\left(1-\delta_{i j}\right)\left|\varepsilon_{j, v}\right|}
\end{aligned}
$$

For a given function $\tau$, the onsite energies utilized are as listed: 


$$
\begin{aligned}
& \tau=s s \sigma: \varepsilon_{i, \mu}=\varepsilon_{i, s}, \varepsilon_{j, v}=\varepsilon_{j, s} \\
& \tau=\operatorname{sp\sigma }: \varepsilon_{i, \mu}=\varepsilon_{i, s} ;, \varepsilon_{j, v}=\varepsilon_{j, p} \\
& \tau=p s \sigma: \varepsilon_{i, \mu}=\varepsilon_{i, p}, \varepsilon_{j, v}=\varepsilon_{j, s} \\
& \tau=p p \sigma: \varepsilon_{i, \mu}=\varepsilon_{i, p}, \varepsilon_{j, v}=\varepsilon_{j, p} \\
& \tau=p p \pi: \varepsilon_{i, \mu}=\varepsilon_{i, p}, \varepsilon_{j, v}=\varepsilon_{j, p}
\end{aligned}
$$

This completes the necessary modifications to the Hamiltonian and overlap required to describe the heterogeneous system. It has also increased the number of parameters in the overall system. For a 3 element scenario such as a silicon wire doped with phosphorus we have added 3 extra parameters, but for say the same system with hydrogen passivation we would add 6 mixing parameters. For an $\mathrm{N}$ element system we add $\frac{n(n-1)}{2}$ mixing parameters. Each parameter has to be optimized; this can become a cumbersomely large project. It may be of interest to find an alternative integral representation of the overlap that will not require mixing parameters. It may be of interest to then exploit that expression to extract mixing parameters for the Hamiltonian via a nonlinear least squares fitting routine.

\subsubsection{Optimized mixing parameters}

The final optimized mixing parameters are shown in figure 56. Once optimized it is clear that indeed as suspected the best fit was as predicted in 5.1.3, and not too extreme as mentioned in 5.1, i.e., $\delta_{i j}$ is not very near 0 or 1. 


\begin{tabular}{|c|c|}
\hline$\delta_{i j}$ & value \\
\hline$\delta_{B P}$ & 0.37590057 \\
$\delta_{B S i}$ & 0.71702206 \\
$\delta_{P S i}$ & 0.58472552 \\
\hline
\end{tabular}

Figure 56. Optimized mixing parameters.

\subsection{DOPING THE PERPENDICULAR WIRE}

In this section the multi-twin silicon nanowire ${ }^{96}$ (MTSNW) is briefly described, and then two applications are explored. First the wire is doped with boron, second it is doped with both phosphorus and boron combined. An SEM image $^{97}$ of a MTSNW is shown in figure 57 . The image shows that the wire has five-fold symmetry with stacking faults between the wedges. Because of this, a dopant atom may preferentially want to float towards the inside or outside of the wire based upon whether or not that particular atom reduces or increases stress. Extensive analysis was performed on both applications. In the first case an earlier set of boron and silicon parameters were used. In the second case the latest set of silicon, boron and phosphorus parameters are used. It is noted that the homo-lumo gap is the same for the undoped unpassivated MTSNW when using either silicon parameter set. 


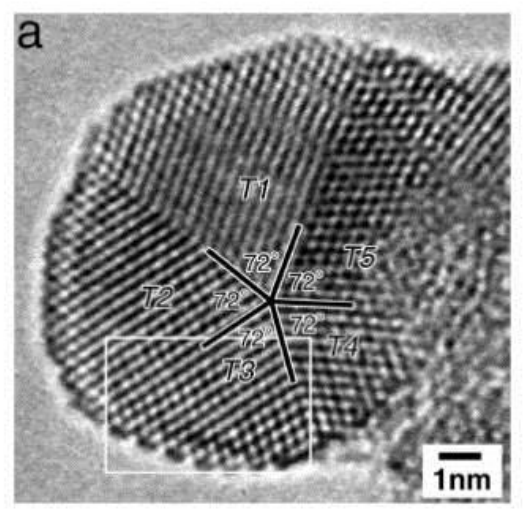

Figure 57. The multi-twinned perpendicular nanowire

There are two different types of MTSNW, parallel and perpendicular. The MTSNW, both parallel and perpendicular cases, have interesting properties. The internal stress of the wire continuously increases ${ }^{96}$ when approaching the center of the core, reaching a maximum near the center. For small diameter wires, less than $6 \mathrm{~nm}$, the MTSNW is lower in energy than the crystalline silicon nanowire.

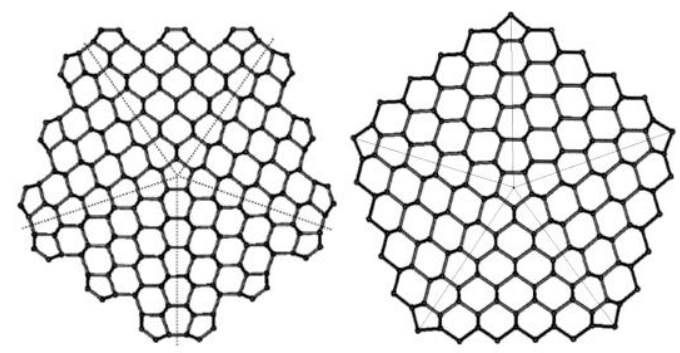

Figure 58. The difference between the parallel (left) and perpendicular (right) wires

The differences in the parallel and perpendicular wire are noted in the above illustration, see figure 58. 


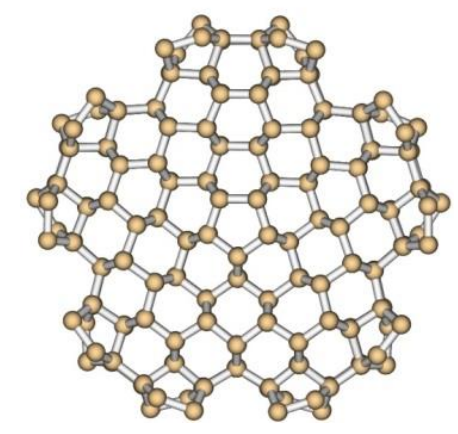

Figure 59. Image of the parallel wire

As the wires get larger in diameter, it was noted that the presence of one dopant atom had less and less influence on the system as a whole. These small energy differences were difficult to capture in the simulation. The lattice constant optimized, unpassivated, parallel, MTSNW is shown in figure 59 for completeness, but was not analyzed. The perpendicular MTSNW was lattice constant optimized, see figure 60 , and in both applications before any analysis was performed. This was done separately for the passivated and unpassivated cases.

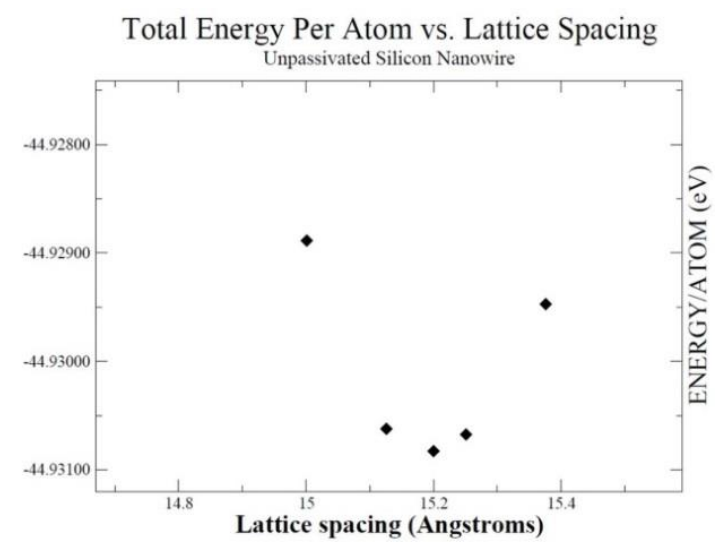

Figure 60. The perpendicular twin wire was lattice optimized 


\subsubsection{Hydrogen passivated multi-twin wire with boron doping}

An early study worth noting was performed by the CMT group and presented at the American Physical Society March Meeting ${ }^{98}$ by Dr. Chakram Jayanthi. This study included an older set of silicon parameters that did not include the function $W_{i \alpha}\left(R_{i j}\right)=W_{i \alpha}^{0} e^{-\alpha_{i \alpha, W} R_{i j}}$. The mixing parameter $\delta_{i j}$ was set to $\delta_{i j}$ $=0.5$ in all cases. A very crude parameter set for hydrogen was used to passivated the wire. The wire is shown in figure 61.

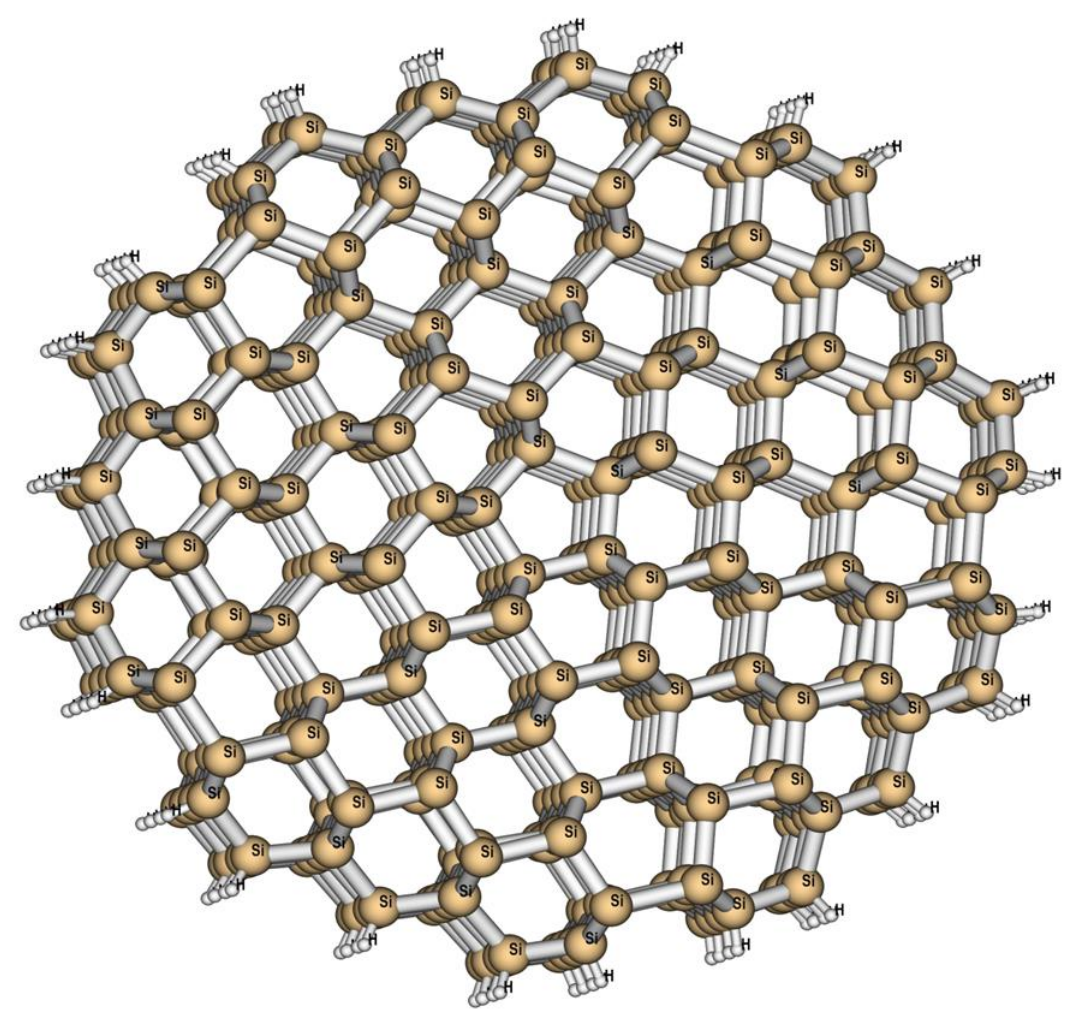

Figure 61. Hydrogen passivated multi-twin wire

Boron was substituted into the wire at every possible location. Due to the 5 fold symmetry, there are 5 wedges. Each wedge has mirror symmetry. This reduces the number of substitutional cases of interest to approximately 7 locations. In 
each of these 7 locations, one at a time, a boron atom was substituted. The boron parameters used were also an older set that did not contain the function $W_{i \alpha}\left(R_{i j}\right)=W_{i \alpha}^{0} e^{-\alpha_{i \alpha, W} R_{i j}}$. The boron and silicon sets at that time had both been tested to be quite good, later they were improved for increased transferability and reliability. The electronegativity of hydrogen is about the same as that of phosphorus, so it is expected that there would be some charge transfer but not a drastic amount like we may see from fluorine.

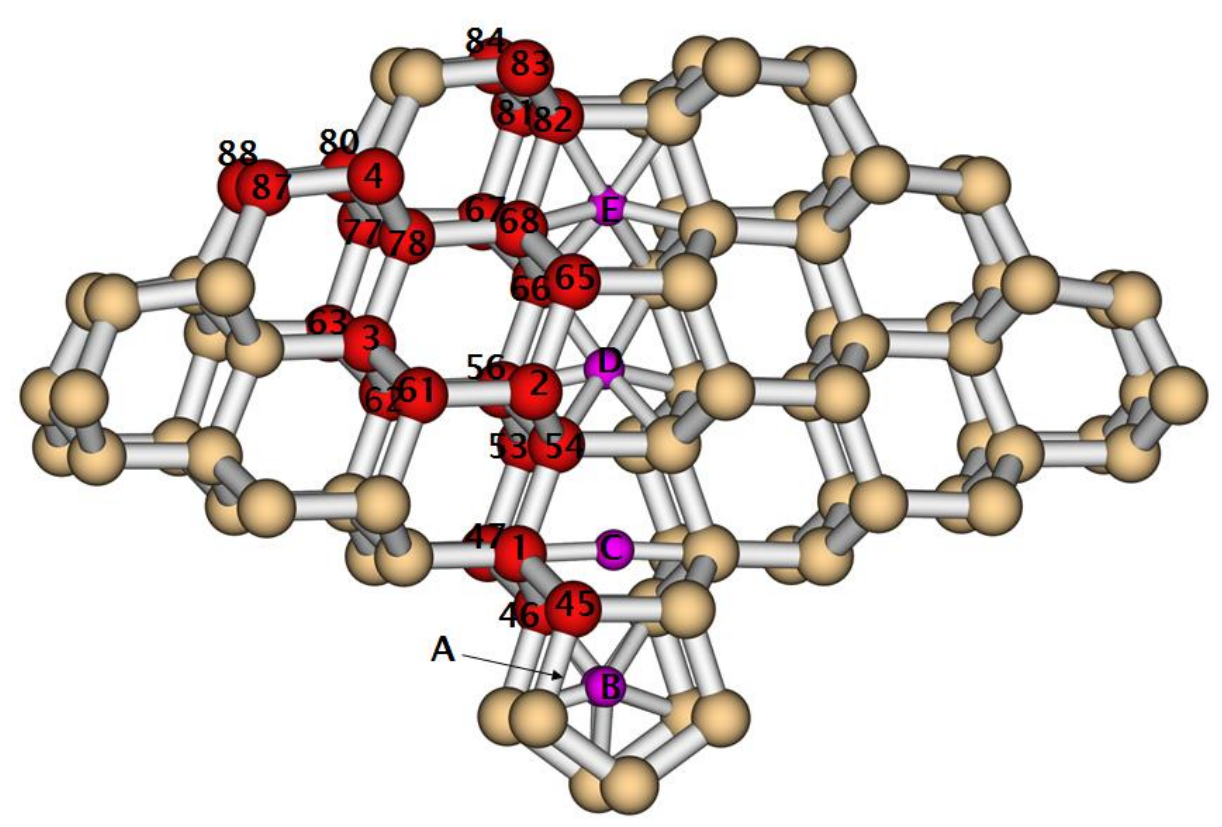

Figure 62. Locations of boron substitution and interstitial placement.

A second case was also performed; interstitial atoms were placed in the channels between the wedges. The wire used is 2 unit cells thick, $2.2 \mathrm{~nm}$ in diameter, and contains 460 silicon atoms and 100 hydrogen atoms. One boron atom was placed at each location, the system was relaxed, and the energy and homo-lumo gap were extracted. See figure 62. Interstitial locations are purple 
and labelled alphabetically, substitutional locations are labeled numerically. The results are listed in figure 63.

\begin{tabular}{|c|c|c|c|c|c|}
\hline location & $\begin{array}{l}\text { binding } \\
\text { energy }(\mathrm{eV})\end{array}$ & $\begin{array}{l}\text { homo- } \\
\text { lumo } \\
\text { gap }(e V)\end{array}$ & location & $\begin{array}{c}\text { binding } \\
\text { energy } \\
(\mathrm{eV})\end{array}$ & $\begin{array}{l}\text { homo- } \\
\text { lumo } \\
\text { gap }(e V)\end{array}$ \\
\hline $\begin{array}{c}\text { passivated } \\
\text { undoped }\end{array}$ & -5.1528 & 0.425 & $\begin{array}{c}\text { unpassivated } \\
\text { undoped }\end{array}$ & -4.7906 & 0.120 \\
\hline 87 & -5.15160 & 0.338 & C & -5.15021 & 0.029 \\
\hline 88 & -5.15159 & 0.341 & $E$ & -5.15007 & 0.200 \\
\hline 83 & -5.15155 & 0.308 & D & -5.15002 & 0.116 \\
\hline 84 & -5.15153 & 0.318 & B & -5.14993 & 0.021 \\
\hline 78 & -5.15125 & 0.259 & A & -5.14951 & 0.152 \\
\hline 46 & -5.15105 & 0.165 & & & \\
\hline 45 & -5.15102 & 0.159 & & & \\
\hline
\end{tabular}

Figure 63. Results of doped multi-twin wire

It is noted that the lowest system energy substitutional location is at the surface. This is because near the surface the reconstruction in the neighborhood around the boron atom is least pronounced as shown in figure 64 .

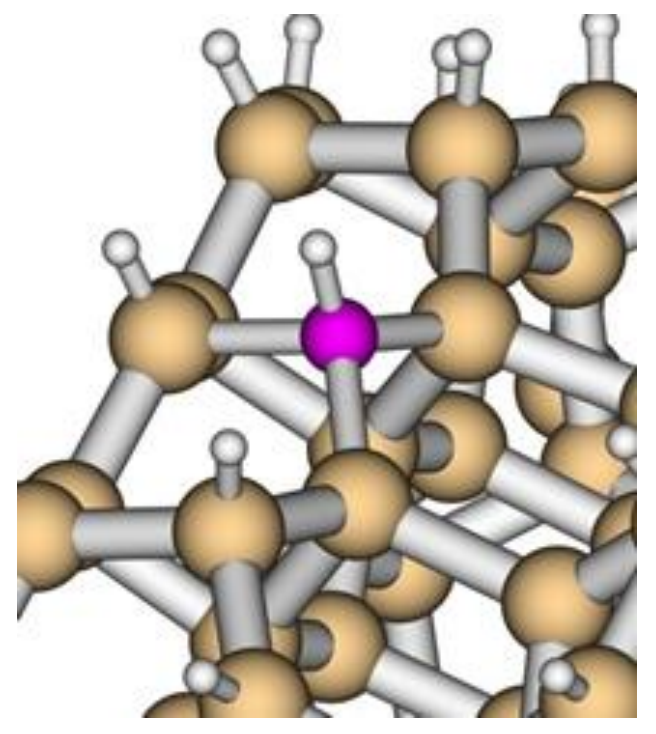

Figure 64. Substitution of boron near the surface. 
It is worth noting that the energy of the system decreases as the boron substituted atom moves away from the center. This is because less stress is induced near the surface. The homo-lumo gap also decreases as the boron atom is moved near the surface, this also makes sense physically.

The energy differences in the system are very small for any case. Given this it would be difficult to say that any location would be preferred over any other location in the substitutional case. The same is approximately true for the interstitial case, although there is a lowest energy location. This location is at a special location in the faulting stack with the most room. Likewise the interstitial case closes the homo-lumo gap and the wire effectively becomes metallic.

Total Density of States of Multiply Twinned Silicon Nanowires Boron at interstial Site C and substitutional site 45

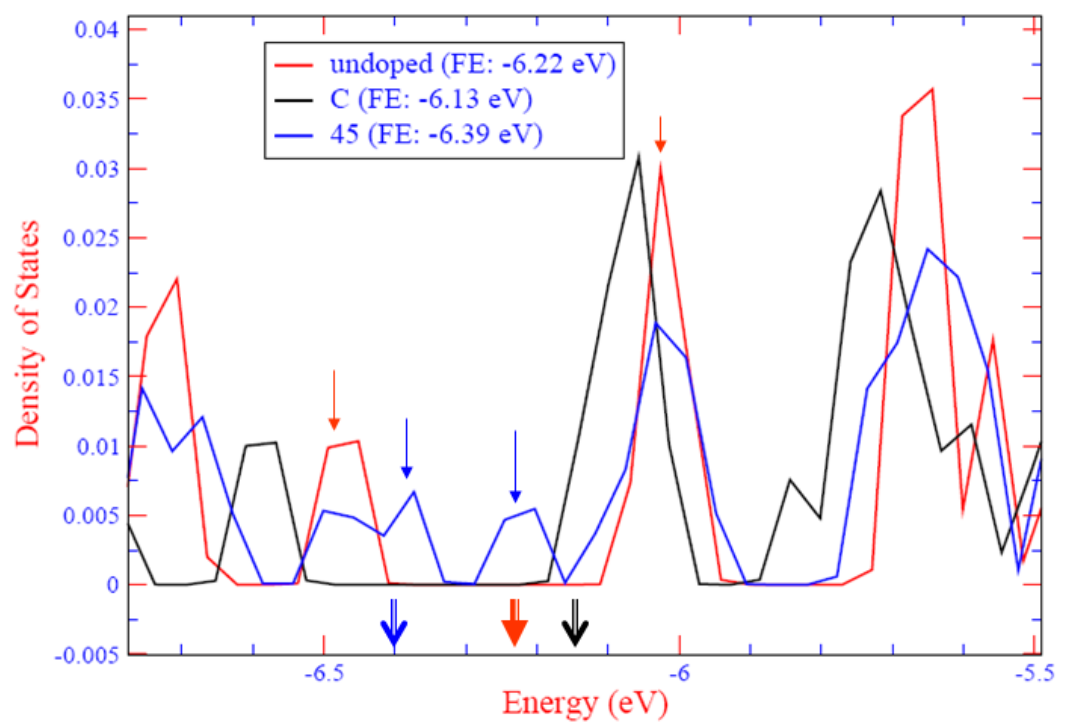

Figure 65. EDOS plot of lowest energy substitutional and interstitial cases in twin-wire 
From the electronic density of states calculation, see figure 65 , it can be seen that the atoms near the center such as point 45 substitutional and point $C$ interstitial both close the homo-lumo gap. The passivated wire undoped has a homo-lumo gap of $0.425 \mathrm{eV}$, which is on the order of what we may expect from silicon, a bit underestimated.

The unpassivated wire has a homo-lumo gap of $0.12 \mathrm{eV}$, this is also physically reasonable due to surface reconstructions. This value is also the same value found with the latest set of silicon parameters that include the $W_{i \alpha}\left(R_{i j}\right)$ formulation. In conclusion it is noted that although we had a very crude model for hydrogen, and we used older boron and silicon parameter sets, a very good result was found. This validates the fact that even when the robust checks are not fully implemented, in some cases, the parameter sets can be quite useful. 


\subsubsection{Simultaneous Boron and Phosphorus Doping}

The second application brings together the latest boron, phosphorus and silicon parameter sets including the $W_{i \alpha}\left(R_{i j}\right)$ function. This work was presented at the American Physical Society March Meeting ${ }^{71}$. The unpassivated MTSNW was lattice constant optimized. The electronic density of states plot is shown in figure 66. The homo-lumo gap is very small it is approximately $0.125 \mathrm{eV}$. This is expected from the previous application in section 5.2.1.

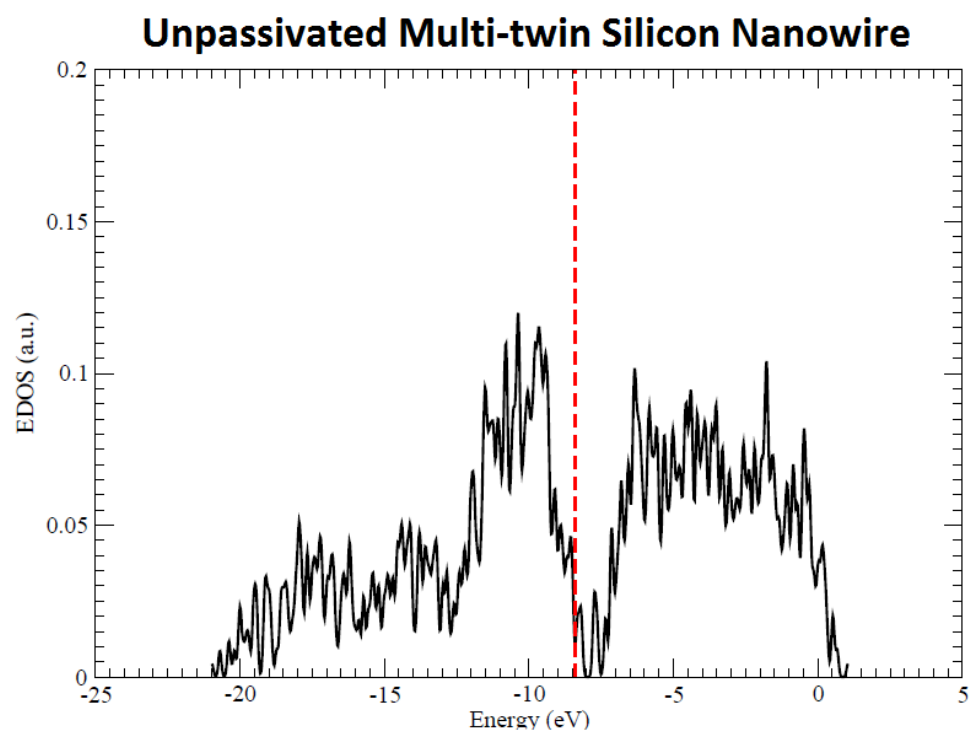

Figure 66. Electronic density of states for the undoped MTSNW

Two cases were examined. The first case is the substitution of a boron atom near the outside of the MTSNW, and a phosphorus atom near the core (Bout). The second case is the exact opposite; the boron is near the core and the phosphorus atom is near the outside of the MTSNW. The first case is shown in figure 67 . 

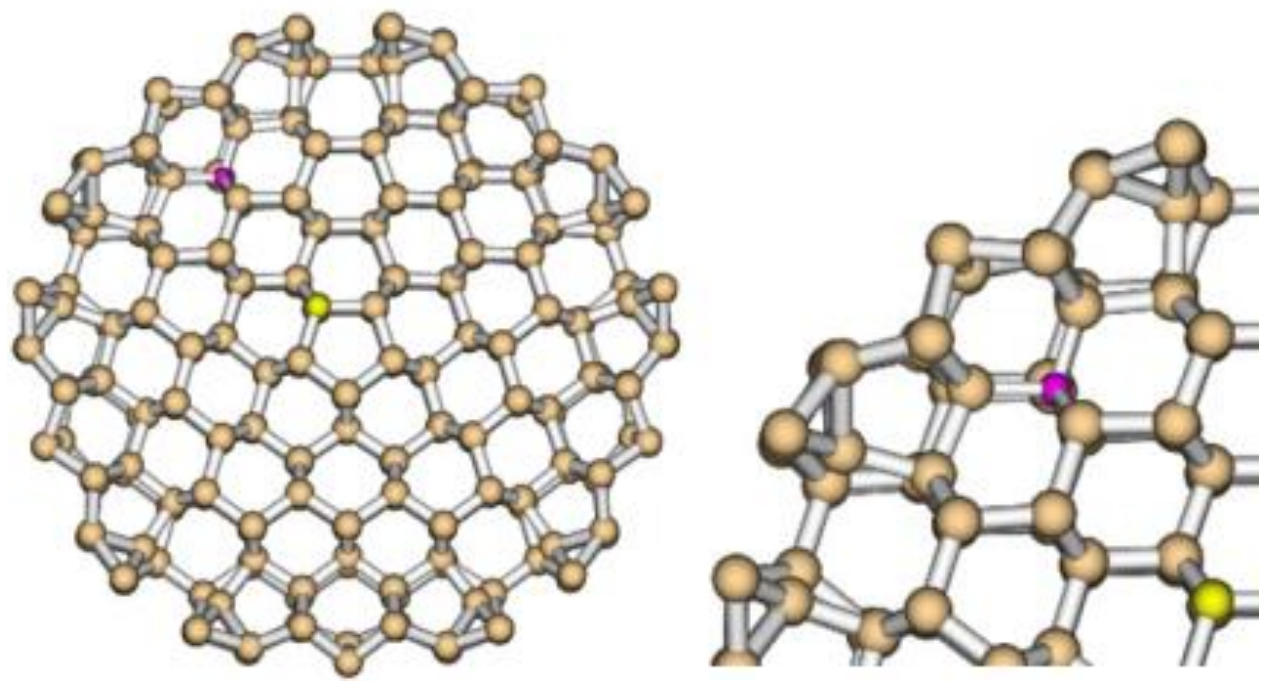

Figure 67. Boron placed outside and phosphorus near the center of the core and enlargement.

The electronic density of states does not look appreciably different since there is a very small gap to begin with. Note that the gap in this case went from $0.125 \mathrm{eV}$ to $0.01 \mathrm{eV}$ closing it even further. See figure 68 .

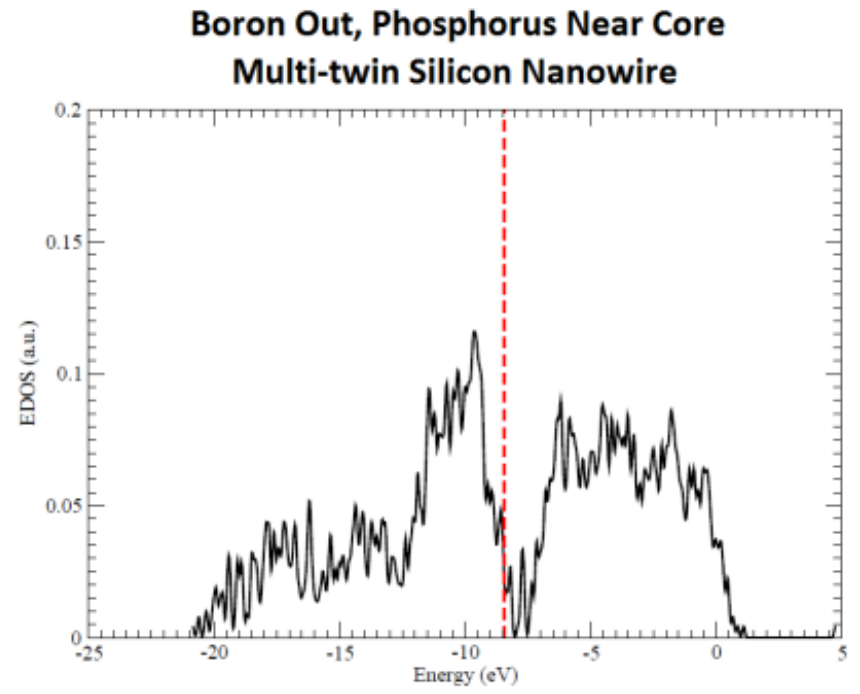

Figure 68. Electronic density of states for the Bout doped MTSNW 
The second case is shown in figure 69 .
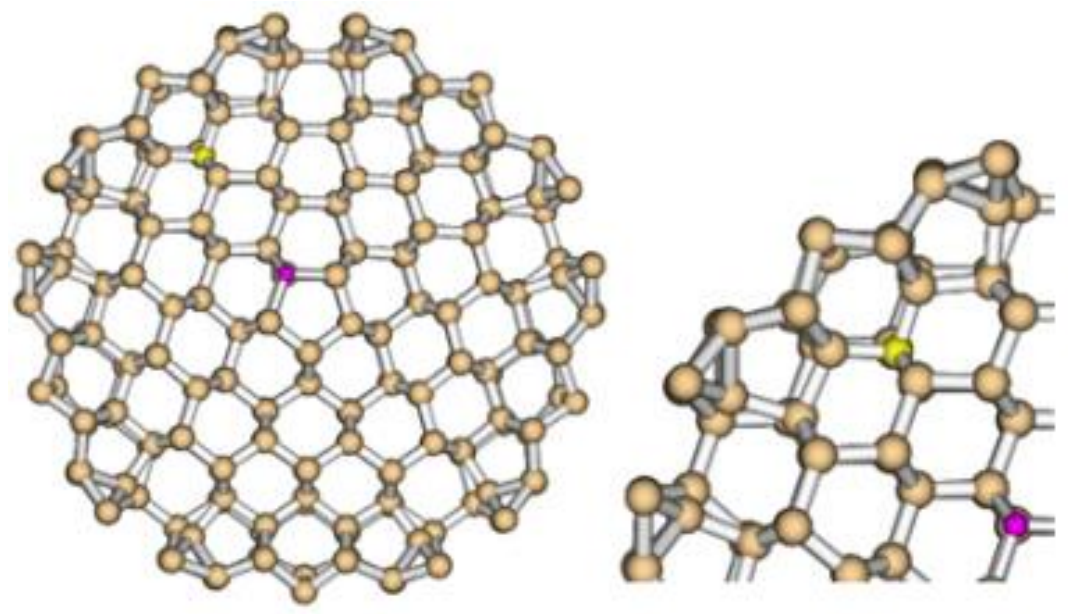

Figure 69. Boron on the inside and phosphorus on the outside

Again the electronic density of states does not look appreciably different. Note that the gap in this case went from $0.125 \mathrm{eV}$ to $0.05 \mathrm{eV}$ cutting the gap in half. See figure 70 .

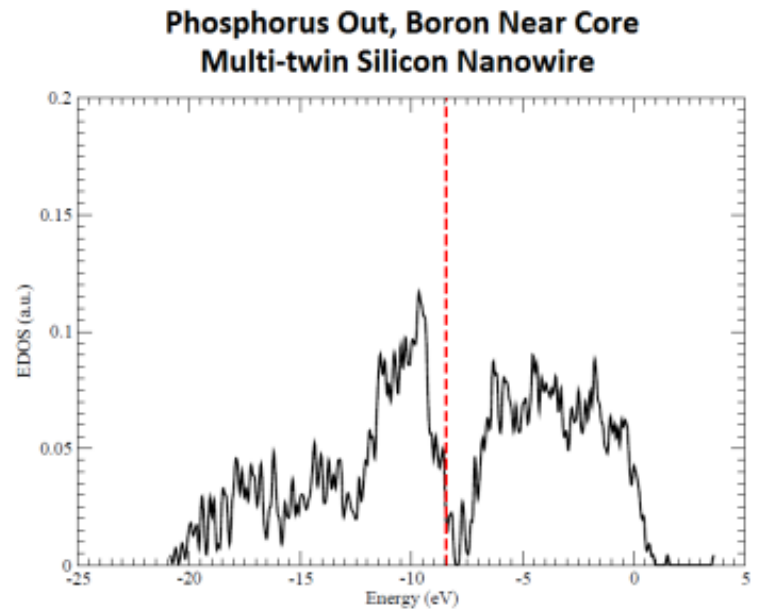

Figure 70. Electronic density of states for the Pout doped MTSNW 
The difference in energy between the Bout and Pout cases are quite small. The Pout case is lower in energy by about $0.001 \mathrm{eV} /$ atom. See figure 71 .

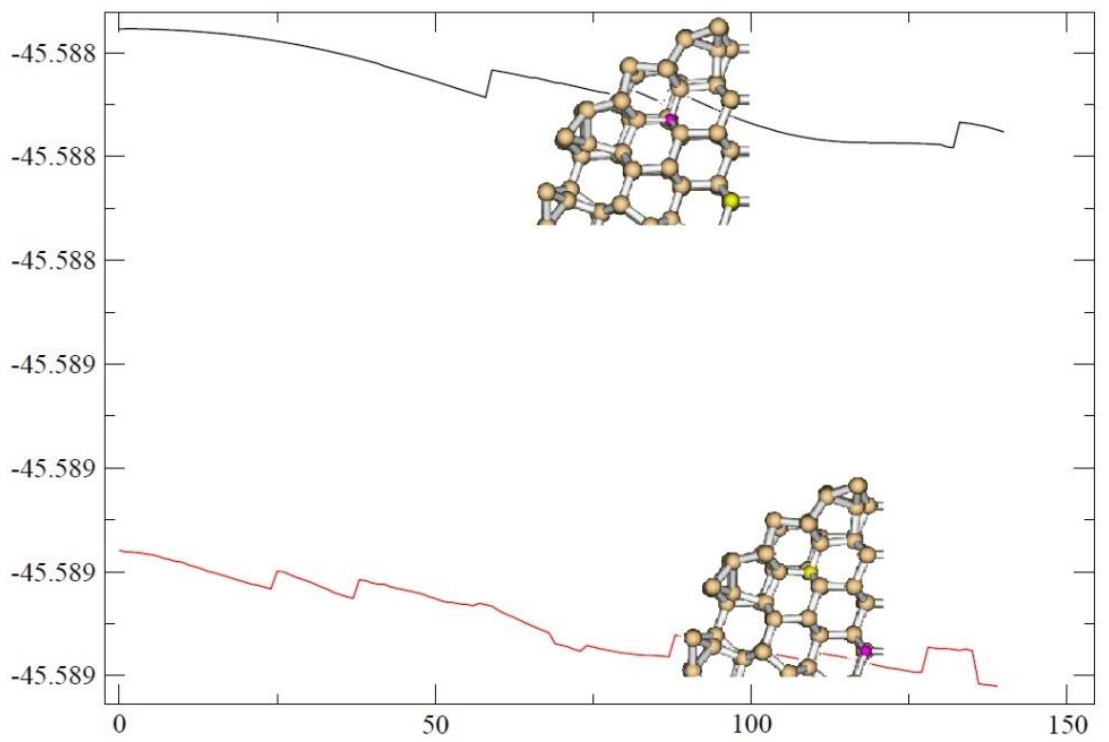

Figure 71. The higher energy state is boron in phosphorus out.

So although the energy difference is small, the result is that the unpassivated MTSNW does have a preference for boron to be near the inside and phosphorus near the outside.

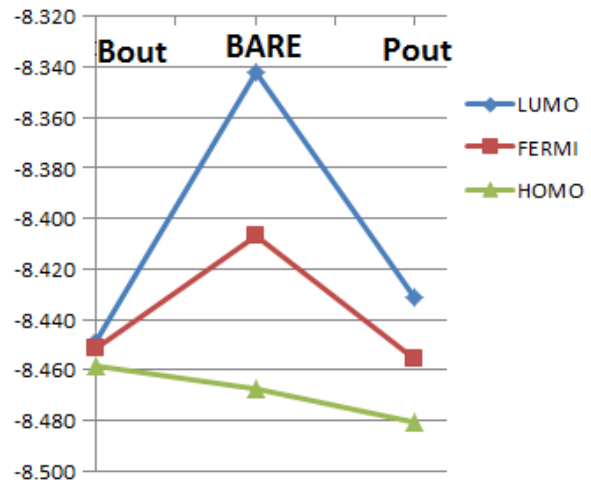

Figure 72. HOMO-LUMO shift due to Bout/Pout swap 
This makes sense because boron is much smaller and puts less stress on the system when inside, and phosphorus is much larger and puts less stress on the system when near the outside of the wire. The homo-lumo shifts and Fermi energy shifts are shown in figure 72 .

\section{Multi-twin Silicon Nanowire Bout/Pout Doped and Undoped Pair Distribution Function}

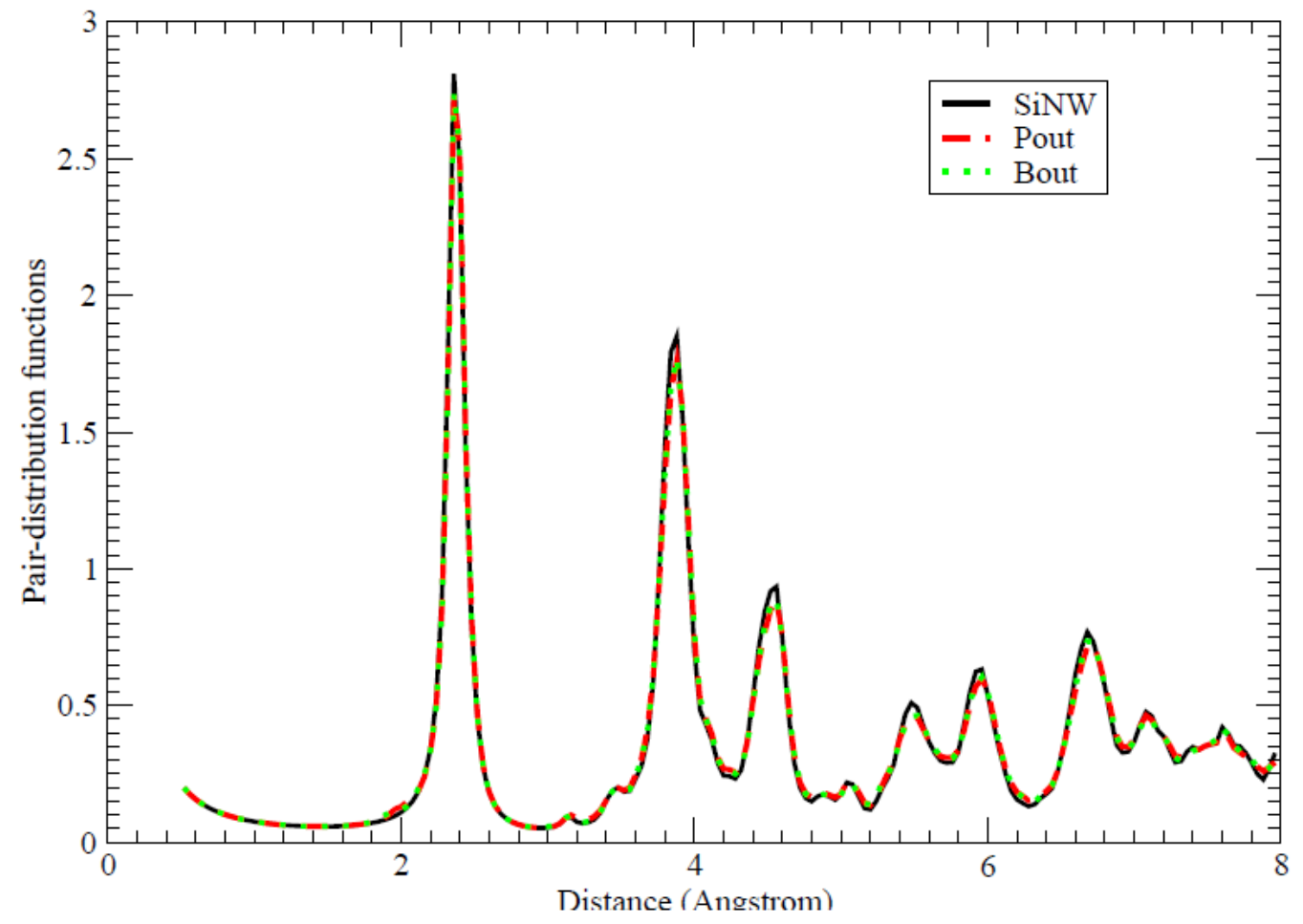

Figure 73. The pair distribution function.

The pair distribution function is not appreciably affected by either doping scheme.

See figure 73. But, the angle distribution function shows an interesting feature. Most angles in the system are around 110 degrees indicating tetrahedral quality, but there is a significant distribution of approximately 60 degrees in the system. 


\section{Multi-twin Silicon Nanowire}

Bout/Pout Doped and Undoped

\section{Angle Distribution Function}

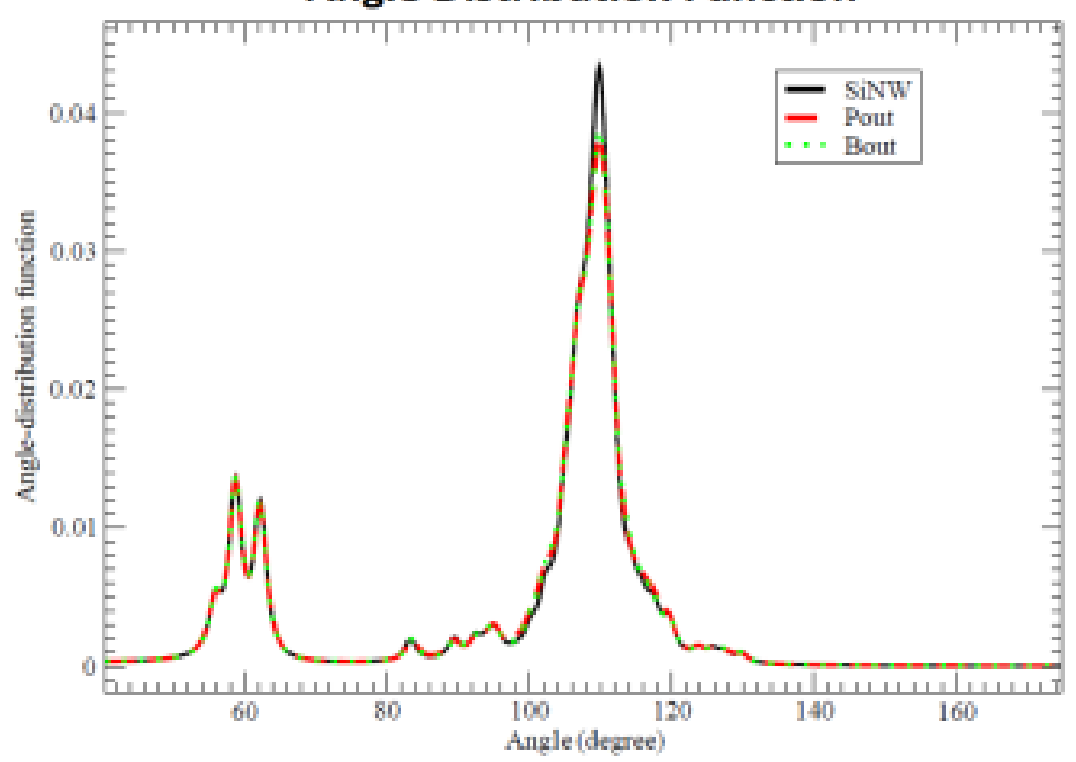

Figure 74. Angle distribution function for the MTSNW

The numerous angles around 60 degrees are easily seen in in figure 74 . They represent surface reconstructions due to no passivation ruining the tetrahedral nature of the wire. These will disappear in with hydrogen passivation, and indeed are not present in figure 61 from the first application.

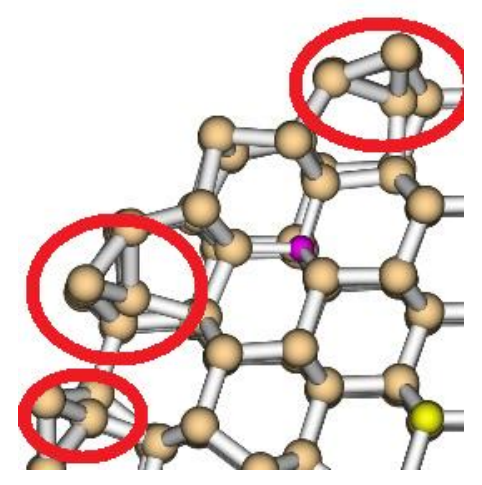

Figure 75. Surface reconstruction in the MTSNW 


\section{CHAPTER VI: SUMMARY AND FUTURE WORK}

\subsection{SUMMARY}

- We have demonstrated a general method to construct a transferable semiempirical Hamiltonian for boron based upon high quality ab-initio methods that is self-consistent and environment dependent.

- The Hamiltonian is in good agreement with DFT for intermediate sized clusters in prediction of energies, bond lengths and bond angles.

- The boron Hamiltonian was utilized to look at relative energies of various intermediate to large clusters.

- Amorphous clusters are competitive with symmetrical clusters up to about 120 atoms, and then compact icosahedral structures without dangling bonds seem to be energetically favorable. Structures with dangling bonds, the boron 80 cage, and large non-compact structures tend to be less energetically favorable.

- We have demonstrated a general method to construct a transferable semiempirical Hamiltonian for phosphorus based upon high quality ab-initio methods that is self-consistent and environment dependent. 
- The Hamiltonian is in good agreement with DFT for intermediate sized clusters in prediction of energies, bond lengths and bond angles.

- We have demonstrated a general method to construct a heterogeneous mixing scheme to construct a Hamiltonian based upon homogeneous parameters and mixing parameters only.

- The phosphorus Hamiltonian is demonstrated to work well with similarly formulated boron and silicon Hamiltonians in comparison to DFT (GGA) in a realistic 460 atom simulation of the silicon multi-twinned wire.

\subsection{FUTURE WORK}

Future work will be focused on applications of the boron and phosphorus Hamiltonian and continued improvement of the SCED-LCAO methodology.

The boron parameters developed in this work will be used to study the stability of one dimensional boron 12 icosahedral chains and 2D sheets based also on the boron 12 atom icosahedra. They will also be used in 2D sheets based on boron/carbon/nitrogen alloys for energy gap manipulations for optoelectronics applications. The phosphorus parameters in conjunction with the boron parameters will be used to study the doping effects of arbitrary structures of silicon. Boron carbide and boron nitride structures and devices are also being considered. 


\section{REFERENCE}

${ }^{1}$ C. Leahy, M. Yu, C.S. Jayanthi, and S.Y. Wu, Phys. Rev. B 74, 155408 (2006).

2 E. Hückel, Quantentheoretische Beiträge zum Benzolproblem: die Elektronenkonfiguration des Benzols und verwandter Verbindungen, Zeitschrift für Physik, 70, 204, 1931.

${ }^{3}$ R. Hoffmann and W.N. Lipscomb, The Journal of Chemical Physics 36, 2179 (1962).

${ }^{4}$ Dewar, Michael, Journal of the American Chemical Society, v 107, N 13, 3902-9 (1985).

${ }^{5}$ J.A. Pople, Approximate Molecular Orbital Theory (McGraw-Hill, 1970).

6 A. Tchernatinsky, S. Desai, G.U. Sumanasekera, C.S. Jayanthi, S.Y. Wu, B. Nagabhirava, and B. Alphenaar, Journal of Applied Physics 99, 034306 (2006).

${ }^{7}$ W.Q. Tian, M. Yu, C. Leahy, C.S. Jayanthi, and S.-Y. Wu, Journal of Computational and Theoretical Nanoscience 6, 390 (2009).

${ }^{8}$ M. Yu, C.S. Jayanthi, and S.Y. Wu, Nanotechnology 23, 235705 (2012).

9 M. Yu, S.Y. Wu, and C.S. Jayanthi, Physica E: Low-Dimensional Systems and Nanostructures 42, 1 (2009).

${ }^{10}$ A.D. Lilak, S.K. Earles, K.S. Jones, M.E. Law, and M.D. Giles, in Electron Devices Meeting, 1997. IEDM '97. Technical Digest., International (1997), pp. 493-496.

${ }^{11}$ I. Boustani, Chemical Physics Letters 240, 135 (1995).

${ }^{12}$ H. Kato, K. Yamashita, and K. Morokuma, Chemical Physics Letters 190, 361 (1992).

${ }^{13}$ R. Hoffmann and W.N. Lipscomb, The Journal of Chemical Physics 37, 2872 (1962).

${ }^{14}$ A.V. Nemukhin and F. Weinhold, The Journal of Chemical Physics 98, 1329 (1993).

15 Leahy, Self-Consistent and Environment-Dependent Hamiltonian for QuantumMechanics Materials Simulations., University of Louisville, 2007.

16 Tchernatinsky, Alexander, Development and Applications of the State of the Art Techniques for Materials Simulation, University of Louisville, 2005.

${ }^{17}$ Mann, J.B., Los Alamos Scientific Laboratory Report (1967).

${ }^{18}$ W.A. Harrison, Phys. Rev. B 31, 2121 (1985).

${ }^{19}$ J. Hubbard, Proceedings of the Royal Society of London. Series A, Mathematical and Physical Sciences 276, 238 (1963).

${ }^{20}$ M. Wolfsberg and L. Helmholz, The Journal of Chemical Physics 20, 837 (1952).

${ }^{21}$ R. Hoffmann, The Journal of Chemical Physics 39, 1397 (1963).

${ }^{22}$ G. Blyholder and C. Coulson, Theoretical Chemistry Accounts: Theory, Computation, and Modeling (Theoretica Chimica Acta) 10, 316 (1968).

${ }^{23}$ H. Dekura, K. Shirai, and A. Yanase, Phys. Rev. B 84, 094117 (2011).

${ }^{24}$ A.B. Anderson and R. Hoffmann, The Journal of Chemical Physics 60, 4271 (1974).

${ }^{25}$ Z. Wang, J. Xiao, and X. Li, Solid State Communications 152, 64 (2012).

${ }^{26}$ X.-Q. Wang, Physical Review B 82, (2010).

${ }^{27}$ J. Wang, Y. Liu, and Y.C. Li, ChemPhysChem 10, 3119 (2009). 
${ }^{28}$ M. J. Frisch, et al, GAUSSIAN 03 (Gaussian, Inc., Wallingford, CT, 2003).

${ }^{29}$ Paul Turner and Grace Development Team, XMGRACE (Portland, OR, 2008).

${ }^{30}$ J. Widany, T. Frauenheim, T. Köhler, M. Sternberg, D. Porezag, G. Jungnickel, and G. Seifert, Phys. Rev. B 53, 4443 (1996).

${ }^{31}$ Rogue Wave Software, IMSL Library (2010).

${ }^{32}$ G. Kresse and J. Hafner, VASP (1993).

${ }^{33}$ M. Atiş, C. Özdoğan, and Z.B. Güvenç, International Journal of Quantum Chemistry 107, 729 (2007).

${ }^{34}$ T.B. Tai, D.J. Grant, M.T. Nguyen, and D.A. Dixon, J. Phys. Chem. A 114, 994 (2009).

${ }^{35}$ D.-M. Li, Z.-H. Xiong, Q.-X. Wan, G.-D. Liu, W.-R. Zhang, and Z. Ren, in (2008), p. $72790 \mathrm{~K}-72790 \mathrm{~K}-8$.

${ }^{36}$ E.K. Yildirim and Z.B. Güvenç, International Journal of Hydrogen Energy 34, 4797 (2009).

${ }^{37}$ R.N. Grimes, J. Chem. Educ. 81, 657 (2004).

${ }^{38}$ M. Böyükata and Z.B. Güvenç, Journal of Alloys and Compounds 509, 4214 (2011).

${ }^{39}$ P. Tandy, C. Leahy, J. Simrall, Y. Ming, C. Jayanthi, and S.-Y. Wu, in APS March Meeting Abstracts (2008), p. 1110.

${ }^{40}$ P. Tandy, M. Yu, C. Leahy, W.Q. Tian, S.Y. Wu, and C.S. Jayanthi, in Bulletin of the American Physical Society (American Physical Society, 2009).

${ }^{41}$ Dunning Thomas, The Journal of Chemical Physics 90, 1007 (1989).

${ }^{42}$ M. Menon and K.R. Subbaswamy, Phys. Rev. B 47, 12754 (1993).

43 T. Frauenheim, G. Seifert, M. Elsterner, Z. Hajnal, G. Jungnickel, D. Porezag, S. Suhai, and R. Scholz, Physica Status Solidi (b) 217, 41 (2000).

${ }^{44}$ N. Bernstein and E. Kaxiras, Phys. Rev. B 56, 10488 (1997).

${ }^{45}$ M.J. Mehl and D.A. Papaconstantopoulos, Phys. Rev. B 54, 4519 (1996).

${ }^{46}$ D.B. Sullenger, K.D. Phipps, P.W. Seabaugh, C.R. Hudgens, D.E. Sands, and J.S. Cantrell, Science 163, 935 (1969).

${ }^{47}$ X. Zhu and X.C. Zeng, The Journal of Chemical Physics 118, 3558 (2003).

${ }^{48}$ A. Fura, F. Tureček, and F.W. McLafferty, International Journal of Mass Spectrometry 217, 81 (2002).

${ }^{49}$ R.J. Nelmes, J.S. Loveday, D.R. Allan, J.M. Besson, G. Hamel, P. Grima, and S. Hull, Phys. Rev. B 47, 7668 (1993).

50 J. Yang, Y. Yang, S.W. Waltermire, X. Wu, H. Zhang, T. Gutu, Y. Jiang, Y. Chen, A.A. Zinn, R. Prasher, T.T. Xu, and D. Li, Nat Nano 7, 91 (2012).

51 W. Gao, Crystal Growth of Alpha-Rhombohedral Boron, Thesis, Kansas State University, 2010.

${ }^{52}$ H.T. Pham, L.V. Duong, B.Q. Pham, and M.T. Nguyen, Chemical Physics Letters 577, 32 (2013).

53 NIST, Computational Chemistry Comparison and Benchmark DataBase:Bad Calculated Geometries (2013).

${ }^{54}$ A.D. Becke, Phys. Rev. A 38, 3098 (1988).

55 A.D. Becke, The Journal of Chemical Physics 98, 1372 (1993).

${ }^{56}$ C. Lee, W. Yang, and R.G. Parr, Phys. Rev. B 37, 785 (1988).

${ }^{57}$ H.G. KüMmel, International Journal of Modern Physics B 17, 5311 (2003).

${ }^{58}$ P.A. Denis, Chemical Physics Letters 395, 12 (2004). 
${ }^{59}$ H. Tang and S. Ismail-Beigi, Phys. Rev. B 82, 115412 (2010).

${ }^{60}$ H. Tang and S. Ismail-Beigi, Phys. Rev. Lett. 99, 115501 (2007).

${ }^{61}$ H. Tang and S. Ismail-Beigi, Phys. Rev. B 80, 134113 (2009).

${ }^{62}$ S. Er, G.A. De Wijs, and G. Brocks, The Journal of Physical Chemistry C 113, 18962 (2009).

${ }^{63}$ K.C. Lau, R. Pati, R. Pandey, and A.C. Pineda, Chemical Physics Letters 418, 549 (2006).

${ }^{64}$ R.R. Zope and T. Baruah, Chemical Physics Letters 501, 193 (2011).

${ }^{65}$ K.C. Lau and R. Pandey, J. Phys. Chem. C 111, 2906 (2007).

${ }^{66}$ J. Russell and R. Cohn, Star of David (Book on Demand, 2012).

${ }^{67}$ D. Y. Zubarev and A.I. Boldyrev, Journal of Computational Chemistry 28, 251 (2007).

${ }^{68}$ S. Mukhopadhyay, H. He, R. Pandey, Y.K. Yap, and I. Boustani, in Journal of Physics: Conference Series (2009), p. 012028.

${ }^{69}$ B. Kiran, S. Bulusu, H.J. Zhai, S. Yoo, X.C. Zeng, and L.S. Wang, Proceedings of the National Academy of Sciences of the United States of America 102, 961 (2005).

${ }^{70}$ N.G. Szwacki, Nanoscale Research Letters 3, 49 (2007).

${ }^{71}$ P. Tandy, C. Leahy, M. Yu, C.S. Jayanthi, and S.Y. Wu, in APS Meeting Abstracts (2011), p. 18015.

${ }^{72}$ P. Tandy, M. Yu, C.S. Jayanthi, and S.-Y. Wu, in APS March Meeting Abstracts (2013), p. 5007.

${ }^{73}$ N. Gonzalez Szwacki, A. Sadrzadeh, and B.I. Yakobson, Phys. Rev. Lett. 98, 166804 (2007).

${ }^{74}$ G. Gopakumar, M.T. Nguyen, and A. Ceulemans, Chemical Physics Letters 450, 175 (2008).

${ }^{75}$ N. Gonzalez Szwacki, A. Sadrzadeh, and B.I. Yakobson, Phys. Rev. Lett. 100, 159901 (2008).

${ }^{76}$ S. De, A. Willand, M. Amsler, P. Pochet, L. Genovese, and S. Goedecker, Phys. Rev. Lett. 106, 225502 (2011).

${ }^{77}$ D.L.V.K. Prasad and E.D. Jemmis, Journal of Molecular Structure: THEOCHEM 771, 111 (2006).

${ }^{78}$ D.A. Drabold, P.A. Fedders, S. Klemm, and O.F. Sankey, Phys. Rev. Lett. 67, 2179 (1991).

${ }^{79}$ P.A. Fedders, Phys. Rev. B 52, 1729 (1995).

${ }^{80}$ R.R. Zope, T. Baruah, K.C. Lau, A.Y. Liu, M.R. Pederson, and B.I. Dunlap, Phys. Rev. B 79, 161403 (2009).

${ }^{81}$ J.T. Muya, F. De Proft, P. Geerlings, M.T. Nguyen, and A. Ceulemans, J. Phys. Chem. A 115, 9069 (2011).

82 R.G. Delaplane, U. Dahlborg, W.S. Howells, and T. Lundström, Journal of NonCrystalline Solids 106, 66 (1988).

${ }^{83}$ M.J. van Setten, M.A. Uijttewaal, G.A. de Wijs, and R.A. de Groot, J. Am. Chem. Soc. 129, 2458 (2007).

${ }^{84}$ M. Widom and M. Mihalkovič, Phys. Rev. B 77, 064113 (2008).

${ }^{85}$ M. Widom and M. Mihalkovič, Journal of Physics: Conference Series 176, 012024 (2009).

${ }^{86}$ M.D. Chen, R.B. Huang, L.S. Zheng, Q.E. Zhang, and C.T. Au, Chemical Physics Letters 325, 22 (2000). 
${ }^{87}$ C. He, J. Shao, R. Shi, and X. Zhu, Computational and Theoretical Chemistry 964, 121 (2011).

${ }^{88}$ M.D. Chen, Q.B. Chen, J. Liu, L.S. Zheng, Q.E. Zhang, and C.T. Au, J. Phys. Chem. A 111, 216 (2007).

${ }^{89}$ R.O. Jones and G. Seifert, The Journal of Chemical Physics 96, 7564 (1992).

90 M.. Chen, R.. Huang, L.. Zheng, and C.. Au, Journal of Molecular Structure: THEOCHEM 499, 195 (2000).

${ }^{91}$ C.D. Zhang, J.C. Lian, W. Yi, Y.H. Jiang, L.W. Liu, H. Hu, W.D. Xiao, S.X. Du, L.L. Sun, and H.J. Gao, J. Phys. Chem. C 113, 18823 (2009).

${ }^{92}$ I. Cabria and J.W. Mintmire, EPL 65, 82 (2004).

93 J.-G. Han and J.A. Morales, Chemical Physics Letters 396, 27 (2004).

${ }^{94}$ M. Yu, C.S. Jayanthi, and S.Y. Wu, Phys. Rev. B 82, 075407 (2010).

${ }^{95}$ M. Yu, C. s. Jayanthi, and S. y. Wu, Journal of Materials Research 28, 57 (2013).

${ }^{96}$ Y. Zhao and B.I. Yakobson, Phys. Rev. Lett. 91, 035501 (2003).

97 M. Takeguchi, M. Tanaka, H. Yasuda, and K. Furuya, Surface Science 493, 414 (2001).

${ }^{98}$ C.S. Jayanthi, P. Tandy, M. Yu, S.Y. Wu, and Y. Zhao, in APS Meeting Abstracts (2009), p. 36004.

${ }^{99}$ X. Yang, Y. Ding, and J. Ni, Phys. Rev. B 77, 041402 (2008). 


\section{CURRICULUM VITAE}

NAME Paul Tandy

CONTACT INFO Please contact the Department of Physics, University of Louisville

\section{EDUCATION}

Doctorate of Philosophy, Physics, December 2013

University of Louisville, Louisville, KY

Dissertation: A SEMI-EMPIRICAL HAMILTONIAN FOR BORON, PHOSPHORUS AND COMPOUNDS CONTAINING BORON, PHOSPHORUS AND SILICON

Master of Science, Electrical Engineering, December 2010

University of Louisville, Louisville, KY

Master of Science, Physics, May 2007

University of Louisville, Louisville, KY

Bachelor of Science, Physics, May 2007

University of Louisville, Louisville, KY 


\section{RESEARCH EXPERIENCE}

- $\quad$ Physicist, Department of Defense, 2012-present

- $\quad$ Research Fellow, University of Louisville, 2008-2012

- $\quad$ Research Assistant, University of Louisville, 2007-2008

\section{TEACHING EXPERIENCE}

Instructor, Department of Physics, University of Louisville, August 2004 2007

Designed the undergraduate educational experiments and taught the laboratory sections for:

- $\quad$ Modern Physics;

- $\quad$ Atomic and Nuclear Physics; and

- $\quad$ Electricity and Magnetism.

\section{AWARDS}

- John A. Dillon Fellowship in Physics, 2008-2012

- $\quad$ The Manual Schwartz Award, 2006

\section{Professional Societies}

- $\quad$ American Physical Society (APS)

- $\quad$ American Society of Mechanical Engineers (ASME)

- $\quad$ American Nuclear Society (ANS)

- $\quad$ Institute of Electrical and Electronics Engineers (IEEE) 


\section{National Meeting Presentations}

The Structure, Stability, and Properties of a One-Dimensional $\alpha$-Boron

Structure

Cherno Baba Kah, P. Tandy, M. Yu, S.Y. Wu, and C.S. Jayanthi, March Meeting of APS, Baltimore, MD March 2013

Simulations of the Structure and Properties of Large Icosahedral Boron

Clusters Based on a Novel Semi-Empirical Hamiltonian

P. Tandy, M. Yu, S.Y. Wu, C.S. Jayanthi, March Meeting of APS, Baltimore, MD March 2013

Development of a Semi-empirical Hamiltonian for Phosphorus for

Quantum Mechanics Based Simulations of Phosphorous-based

Nanostructures

P. Tandy, M. Yu, C. Leahy, W.Q. Tian, S.Y. Wu, and C.S. Jayanthi, March Meeting of APS, Dallas, TX March 2011

Structural and Electronic Properties of Boron Doped Multiply Twinned Silicon

Nanowires

C.S. Jayanthi, P. Tandy, M. Yu, S.Y. Wu, and Y. Zhao, March Meeting of APS, Pittsburgh, PA,

March 2009. 


\section{Computational Studies of Nanostructures of Boron}

P. Tandy, M. Yu, C. Leahy, W.Q. Tian, S.Y. Wu, and C.S. Jayanthi, March Meeting of APS, Pittsburgh, PA, March 2009

Development of a Semi-empirical Hamiltonian for Boron and the Prediction of

Structures for Intermediate-Size Boron Clusters Computational Studies of

Nanostructures of Boron

P. Tandy, C. Leahy, J. Simrall, W.Q. Tian, Y. Ming, S. Shen, C. S. Jayanthi and S.-Y. Wu, March Meeting of APS, New Orleans, LA, March 2008. 